The University of Maine

DigitalCommons@UMaine

Biology and Ecology Faculty Scholarship

School of Biology and Ecology

2017

Bees of Maine, with a State Species Checklist

Alison C. Dibble

Francis A. Drummond

Constance Stubbs

Michael Veit

John S. Ascher

Follow this and additional works at: https://digitalcommons.library.umaine.edu/bio_facpub

Part of the Animal Studies Commons, Climate Commons, and the Entomology Commons

This Article is brought to you for free and open access by DigitalCommons@UMaine. It has been accepted for inclusion in Biology and Ecology Faculty Scholarship by an authorized administrator of DigitalCommons@UMaine. For more information, please contact um.library.technical.services@maine.edu. 


\title{
Bees of Maine, with a State Species Checklist
}

\author{
Alison C. Dibble ${ }^{1, *}$, Francis A. Drummond ${ }^{1,2}$, Constance Stubbs ${ }^{1,3}$, Michael Veit $^{4}$, \\ and John S. Ascher ${ }^{5}$
}

\begin{abstract}
We present a new county checklist developed from bee research in Maine since the 1800s. The list contains 278 bee species in 37 genera and 6 families, of which all but 8 are native, with $\geq 50$ taxa each in Andrena and Lasioglossum. Data for 16 counties from publications, museum collections, and recent surveys varied in number of species from 8 (Androscoggin) to 197 (Hancock). Research since 1930 on Vaccinium angustifolium (Lowbush Blueberry) led to many records. Twenty-one species are considered unusual, including 3 first recorded in 2016: Epeoloides pilosulus, Melitta melittoides, and Holcopasites calliopsidis. Maine records provide evidence of declines in Bombus affinis, decline in $B$. terricola followed by partial recovery, and increase in B. impatiens. Crops that should be studied regarding associated bees are Malus pumila (Apple), Vaccinium corymbosum (Highbush Blueberry), Vaccinium macrocarpon (American Cranberry), and Curcurbitaceae (cucurbits). Montane, sandy, and island habitats were identified as priorities for future sampling. We discuss records of bee species from New England relevant to understanding the Maine fauna, bee diversity, changes in abundance, cleptoparasitism, pesticide impacts, habitat requirements, and climate change.
\end{abstract}

\section{Introduction}

In Maine, native bees have received attention due to their role as pollinators, in particular of native, insect-dependent Vaccinium angustifolium Aiton (Lowbush Blueberry). This crop is designated in the industry as "wild blueberry" and also known as Low Sweet Blueberry. It is unusual in that large monocultural stands of a wild shrub are managed commercially (Hall et al. 1979). Extensive studies of Lowbush Blueberry since the 1960s (Boulanger et al. 1967; Bushmann and Drummond 2015; Drummond and Stubbs 1997a, 1997b, 2003; Stubbs et al. 1992) have identified that the most important pollinators for this crop are native bees including Bombus (bumble bees), Andrena (mining bees), Halictus and Lasioglossum (sweat bees), Megachile (leaf-cutter bees), and Osmia (mason or orchard bees). Of particular interest to researchers and growers are the alternate forage plants visited by bees for pollen and nectar before and after the bloom period for the Lowbush Blueberry crop (Bushmann and Drummond 2015, Stubbs et al. 1992). Other research foci within the Lowbush Blueberry pollination system are the effects of pesticides, pests, and diseases on native bees (Bushmann et al. 2012, Drummond 2012a).

\footnotetext{
${ }^{1}$ University of Maine, School of Biology and Ecology, 5722 Deering Hall, Orono, ME 04469. ${ }^{2}$ University of Maine, Cooperative Extension, 305 Deering Hall, Orono, ME 04469. ${ }^{3}$ Current address - PO Box 799, Winterport, ME 04496. ${ }^{4} 93$ Chestnut Street, Pepperell, MA 01463. ${ }^{5}$ Department of Biological Sciences, National University of Singapore, 14 Science Drive 4, Singapore 117543. *Corresponding author - adibble2@gmail.com.
}

Manuscript Editor: David Halliwell 
A.C. Dibble, F.A. Drummond, C. Stubbs, M. Veit, and J.S. Ascher

Collecting for purposes other than to understand pollination of Lowbush Blueberry has been less intensive despite the longstanding importance of orchard crops and research on invasive plant pollination since the early 1990s (Stubbs et al. 2007). Procter $(1938,1946)$ included bees in a general survey of the Mount Desert Region, and in recent decades several bee surveys have been conducted that were not related to Lowbush Blueberry (Dibble et al. 1997; Droege 2010; Stubbs et al. 1996, 2007). Diverse bees also visit other native blueberry species including Vaccinium corymbosum L.(Highbush Blueberry), common in Maine wetlands, and also $V$. myrtilloides Michx. (Common Blueberry), which often grows intermixed with Lowbush Blueberry.

In Maine, the study of native bees and their collection has been uneven across time and geographic locale (Table 1). The earliest scientific studies of Maine bees include reports of an entomological collecting trip to northern parts of Penobscot and Piscataquis Counties by Alpheus S. Packard Jr. (1861). There is also a description by Cresson (1863) of 2 currently valid Nomada species from unknown localities in Maine: $N$. depressa and the poorly known N. proxima; both are from the collection of Edward Norton, and their type specimens are deposited in the Academy of Natural Sciences Philadelphia. Another collector, Frederick Allen Eddy, collected bees in the early 1880s from the vicinity of Orono, Penobscot County, many of which are deposited in the University of Maine Collection.

John H. Lovell made the most important historical contribution to the study of Maine bees by documenting the bee fauna of Waldoboro in Lincoln County where he lived and by making broader studies of regional bees and their floral associations (Covell 1972; Lovell 1900, 1905a, 1905b, 1907, 1908, 1910, 1911, 1913, 1922a, 1922b, 1924, 1925a, 1925b, 1925c; Lovell and Cockerell 1905, 1906, 1907a, 1907b; Lovell and Lovell 1932; Pellett 1939). His collections, which consist of about 16,000 specimens of bees and other flower-visiting insects, were brought to the University of Louisville insect collection (renamed as the Lovell Insect Museum). Most of Lovell's type specimens of New England bees, including those from Maine, are in the National Museum of Natural History, with lectotype designations provided by Covell (1972), and have been databased and imaged. However, the types of 4 additional species described from New England by Lovell (1909) and originally deposited in the Museum of the Boston Society of Natural History, were overlooked by Covell (1972). These types are now in the Museum of Comparative Zoology at Harvard University (Moure and Hurd 1987). In all, Lovell described from New England 15 currently valid bee species ( 2 of questionable validity; 8 with coauthor T.D.A. Cockerell) and 18 additional names now placed in synonymy, with Waldoboro the type locality of 10 valid species and an additional 14 taxa now considered to be junior synonyms. Lovell made important contributions to what is known about pollination ecology, flower morphology, and the honey plants of North America (see the biography of John Lovell at https://www.encyclopedia. $\mathrm{com} / \mathrm{doc} / 1 \mathrm{G} 2-2830902685 . \mathrm{html})$. His work was of particular importance as a foundation for regional investigations of floral specialization (oligolecty), complementing investigations of bees in the Midwestern United States by the pioneering 
A.C. Dibble, F.A. Drummond, C. Stubbs, M. Veit, and J.S. Ascher

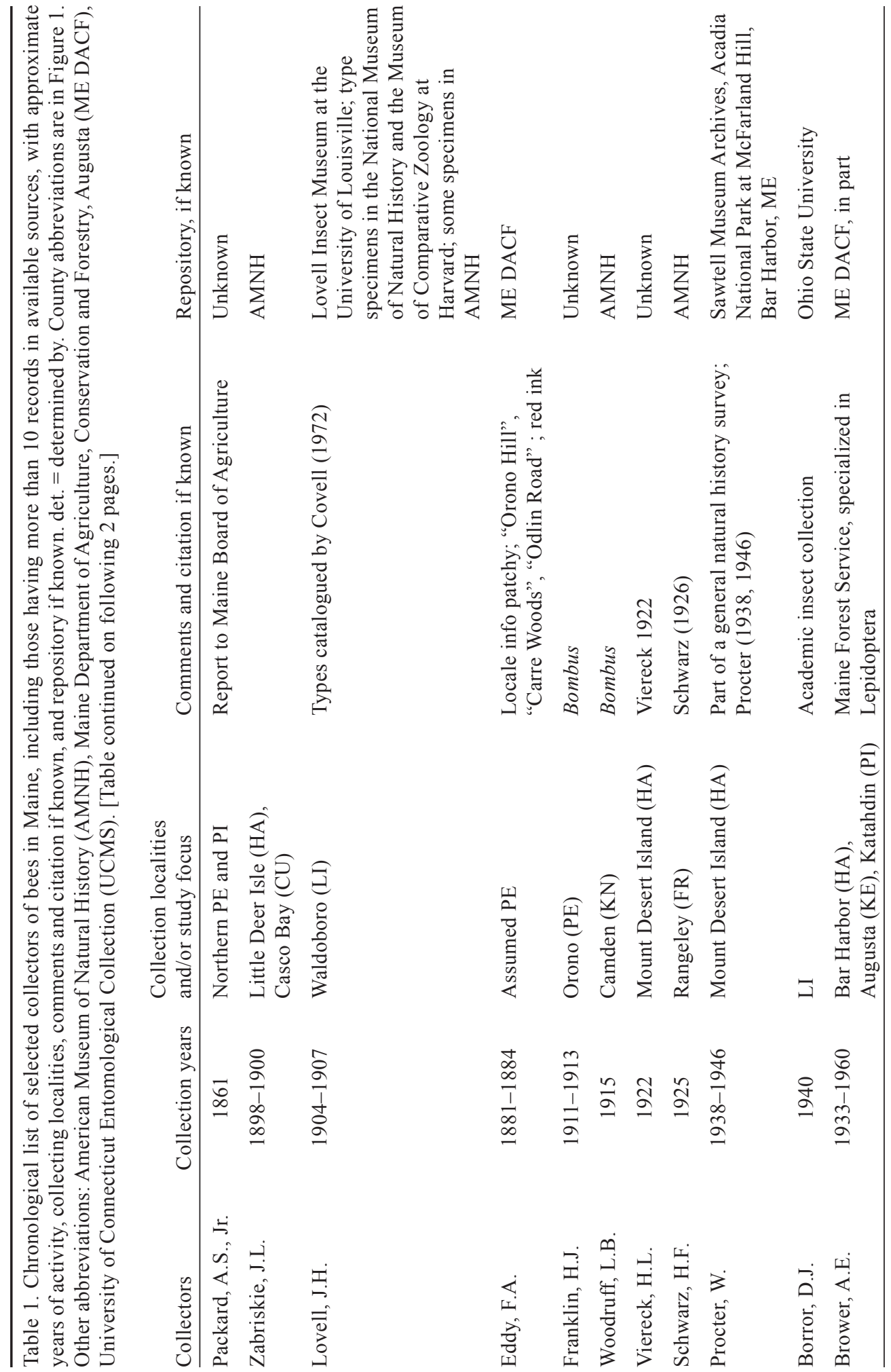


A.C. Dibble, F.A. Drummond, C. Stubbs, M. Veit, and J.S. Ascher

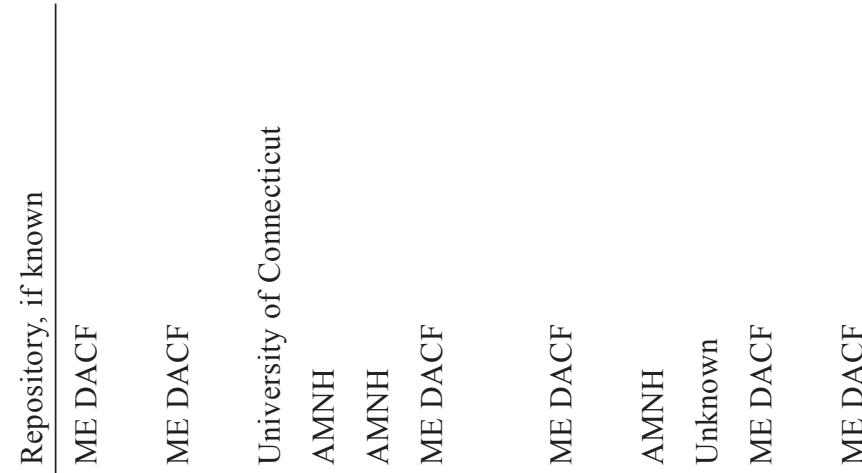

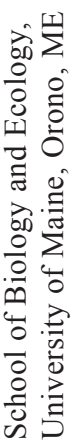

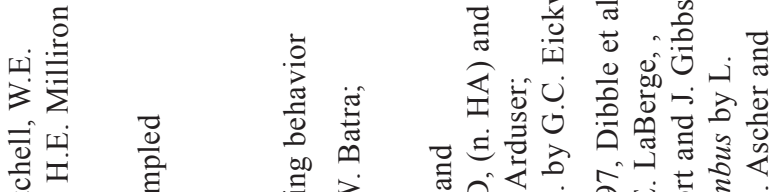

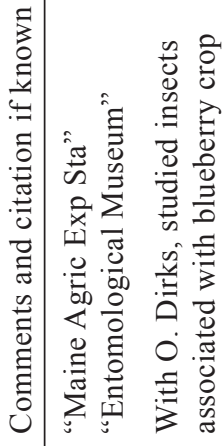

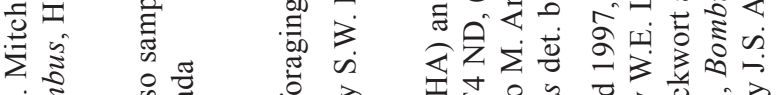

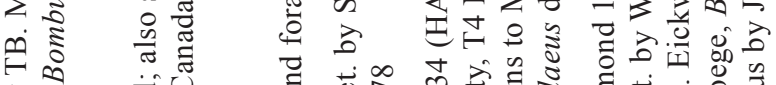

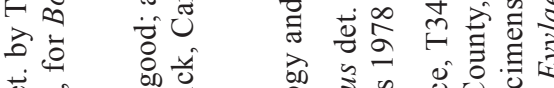

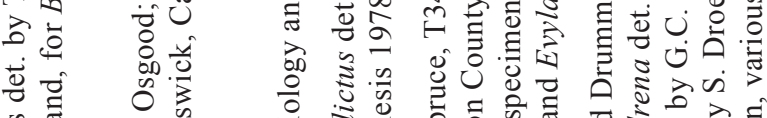

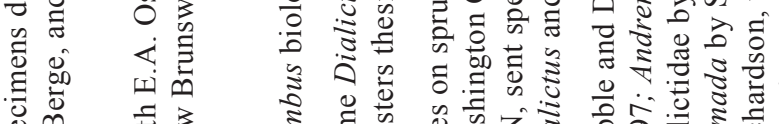

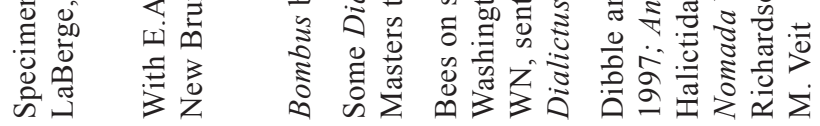

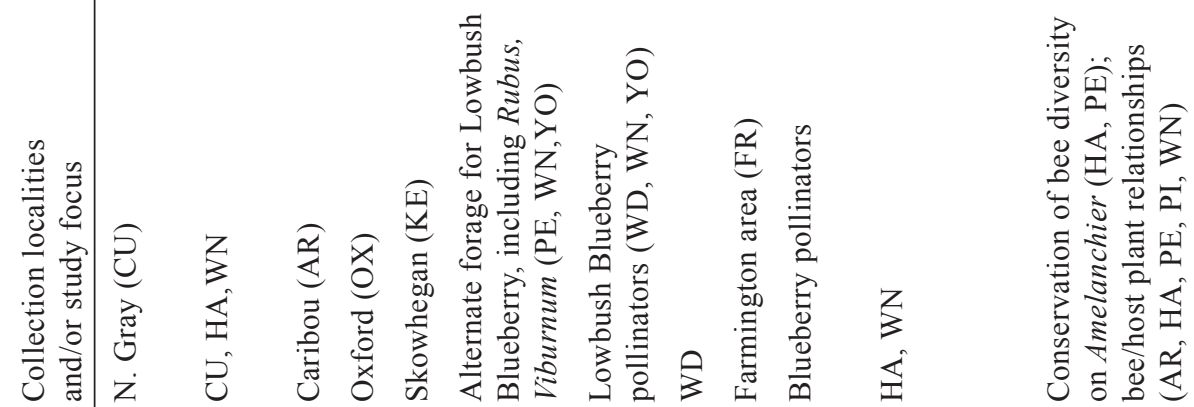

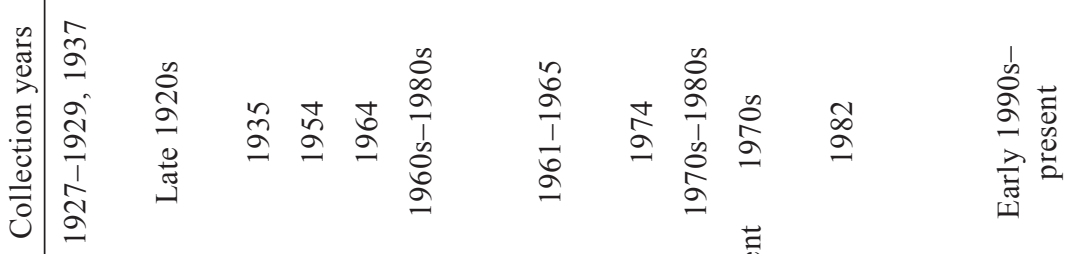

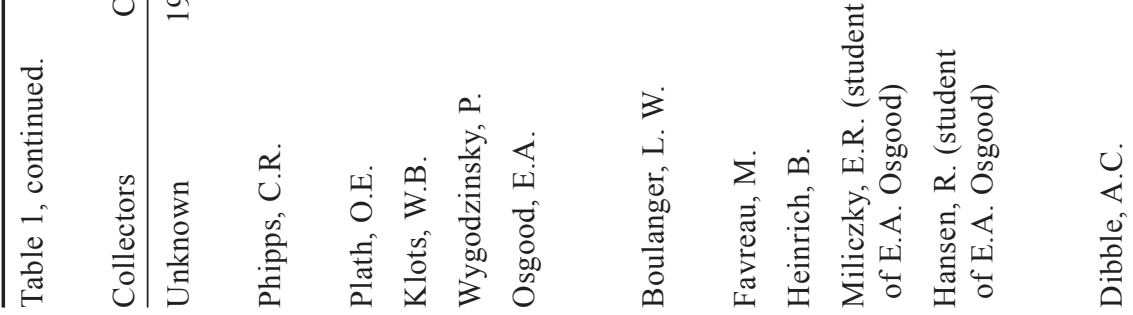


A.C. Dibble, F.A. Drummond, C. Stubbs, M. Veit, and J.S. Ascher

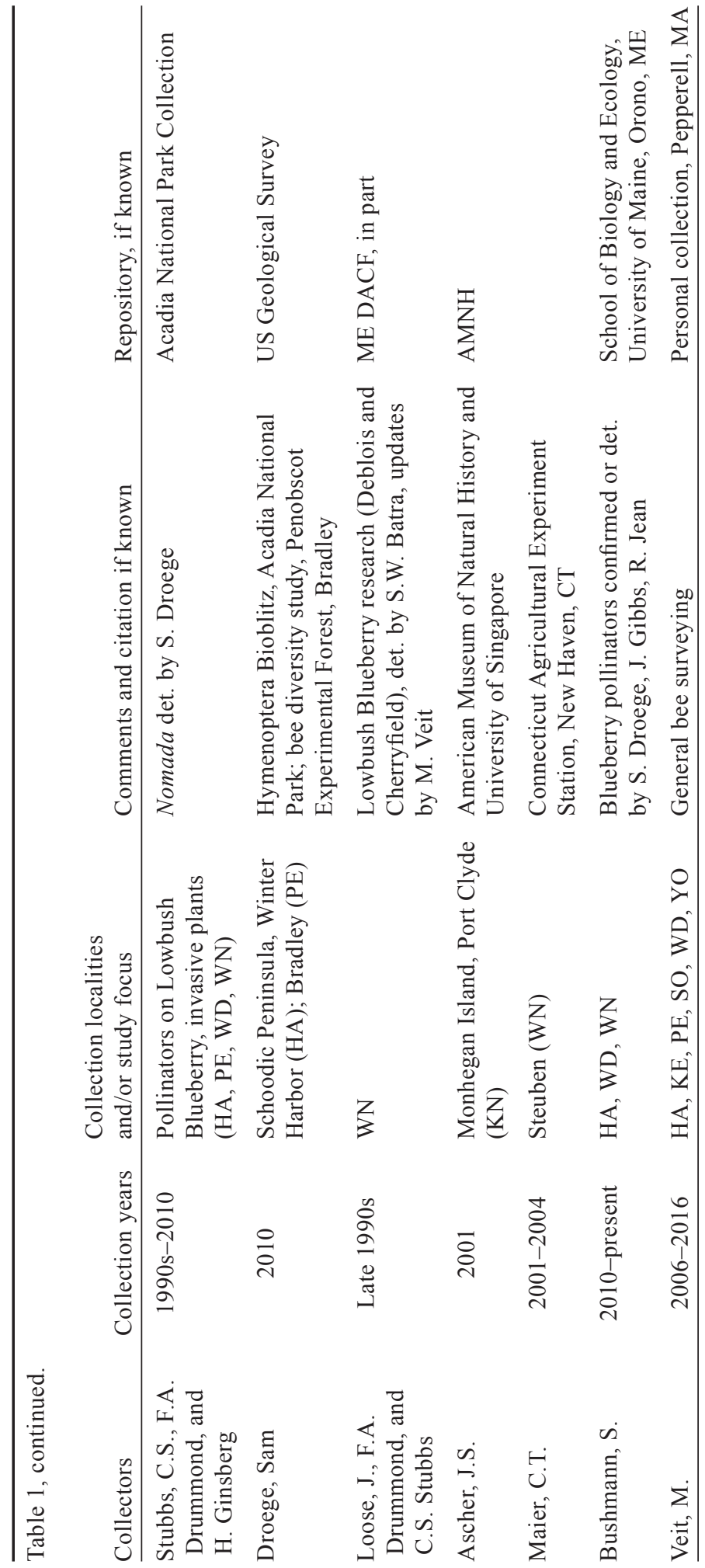


mellitologists Sigmund Graenicher $(1905,1911,1914,1927,1935)$ and Charles Robertson (1929).

Other earlier scientists who contributed to what is known of Maine bees include Franklin $(1911,1912)$, who made the first extensive studies of Maine bumble bees (Bombus sensu lato, including Psithyrus), and cited Orono, ME, among the syntype localities in his description of Psithyrus fernaldae Franklin, 1911. This taxon is now cited as Bombus (Psithyrus) fernaldae or treated as a synonym of the Palearctic B. (P.) flavidus Eversmann (see Cameron et al. 2007). Viereck (1922) described from Mount Desert Island 2 Andrena species now in synonymy. Herbert F. Schwarz (1926) reported bees collected at Rangeley in Franklin County and deposited voucher specimens in the American Museum of Natural History. William Procter $(1938,1946)$ included bees in his general biological survey of Mount Desert Island in Hancock County. In Washington, Hancock, Cumberland, Knox, and Lincoln counties and perhaps elsewhere in the late 1920s, Clarence R. Phipps (1930) inventoried insects associated with blueberry species and Gaylussacia baccata (Wangenh.) K. Koch (Black Huckleberry). Auburn E. Brower, a well-known microlepidopteran specialist, collected Maine bees among other insects from the early 1930s for almost 50 years, in the Augusta area of Kennebec County and many other Maine locales (Davis and Hevel 1995).

Interest in Lowbush Blueberry prompted many research projects since the work of Phipps (1930). From early times, a high priority was given to documenting bee fauna and obtaining expert identification of specimens, as correct determination to the level of species is a crucial aspect in understanding ecological patterns (see discussion in Cane 2001). In 1961-1965, bees and other insect visitors to Lowbush Blueberry were documented in 3 Maine counties and 4 Canadian provinces (Boulanger et al. 1967). Eben A. Osgood $(1972,1989)$ examined the nesting biology of Andrena and contributed to the identification of 2 Osmia species (Rust and Osgood 1993). His students and others extended this research by investigating native plants as floral resources and the response of the bee communities to pesticides applied to control Choristoneura fumiferana (Clemens) (Spruce Budworm) outbreaks (Hansen and Osgood 1983; Miliczky and Osgood 1979a, b; Stubbs et al. 1992, 1996).

Bernd Heinrich has been internationally recognized for his research on the ecological physiology of bee and moth thermoregulation, and for his numerous writings in natural history (Heinrich 1971, 1976b, 1979, 1993, 1994, 1995, 2004; Heinrich and Chavarría 2001; Heinrich and Heinrich 1983a, 1983b; Heinrich et al. 1977). Heinrich conducted important research on the behavior and biology of Bombus in the Farmington, ME, area. Starting in the 1970s, he elucidated the ecological physiology of bumble bee energy budgets for optimal foraging and thermoregulation (Heinrich 1972a, 1972b, 1972c, 1972d, 1973, 1974a, 1974b, 1975, 1976a, 1976c, 1979, 1995, 2004).

These and additional researchers, natural historians, and collectors who have provided baseline information on Maine's bee fauna and bee biology are listed chronologically and annotated in Table 1. Identification of some Maine bee specimens to species was conducted by resident scientists, especially S. Bushmann and 
A.C. Dibble, F.A. Drummond, C. Stubbs, M. Veit, and J.S. Ascher

C.S. Stubbs, and most identifications were by taxonomic specialists on Northeastern bees, including T.B. Mitchell (1960, 1962), G.C. Eickwort, and W.E. LaBerge. More recently the specialists who determined Maine bees have included J. Gibbs, S. Droege, T. Griswold, J.S. Ascher, L. Richardson, and M. Veit.

This report on bee diversity in Maine includes a state checklist of species occurrence by county (Table 2, Fig. 1) based on all available data, including specimens examined by the authors, taxonomic catalogs, revisions, other literature, and digitized specimen records. The latter include specimens from multiple collections in the northeastern United States, including the American Museum of Natural History (AMNH), Cornell University, the University of Connecticut, and other collaborating institutions, compiled using open-source Arthropod Easy Capture (AEC; Seltmann 2013) (see http://biodiversity-informatics-training.org/ wp-content/uploads/2014/03/D2_P6_CW_AEC2.pdf) and made publicly accessible through the biodiversity portals Discover Life (http://www.discoverlife.org)

Figure 1. State of Maine, showing approximate boundaries of the 16 counties, and number of bee species known. County names are abbreviated as: $\mathrm{AN}=$ Androscoggin, $\mathrm{AR}=$ Aroostook, $\mathrm{CU}=$ Cumberland, FR $=$ Franklin, $\mathrm{HA}=$ Hancock, KE = Kennebec, $\mathrm{KN}=$ Knox, LI = Lincoln, $\mathrm{OX}=$ Oxford, $\mathrm{PE}=$ Penobscot, $\mathrm{PI}=$ Piscataquis, SA $=$ Sagadahoc, $\mathrm{SO}=$ Somerset, $\mathrm{WD}=$ Waldo, $\mathrm{WN}=$ Washington, $\mathrm{YO}=$ York.

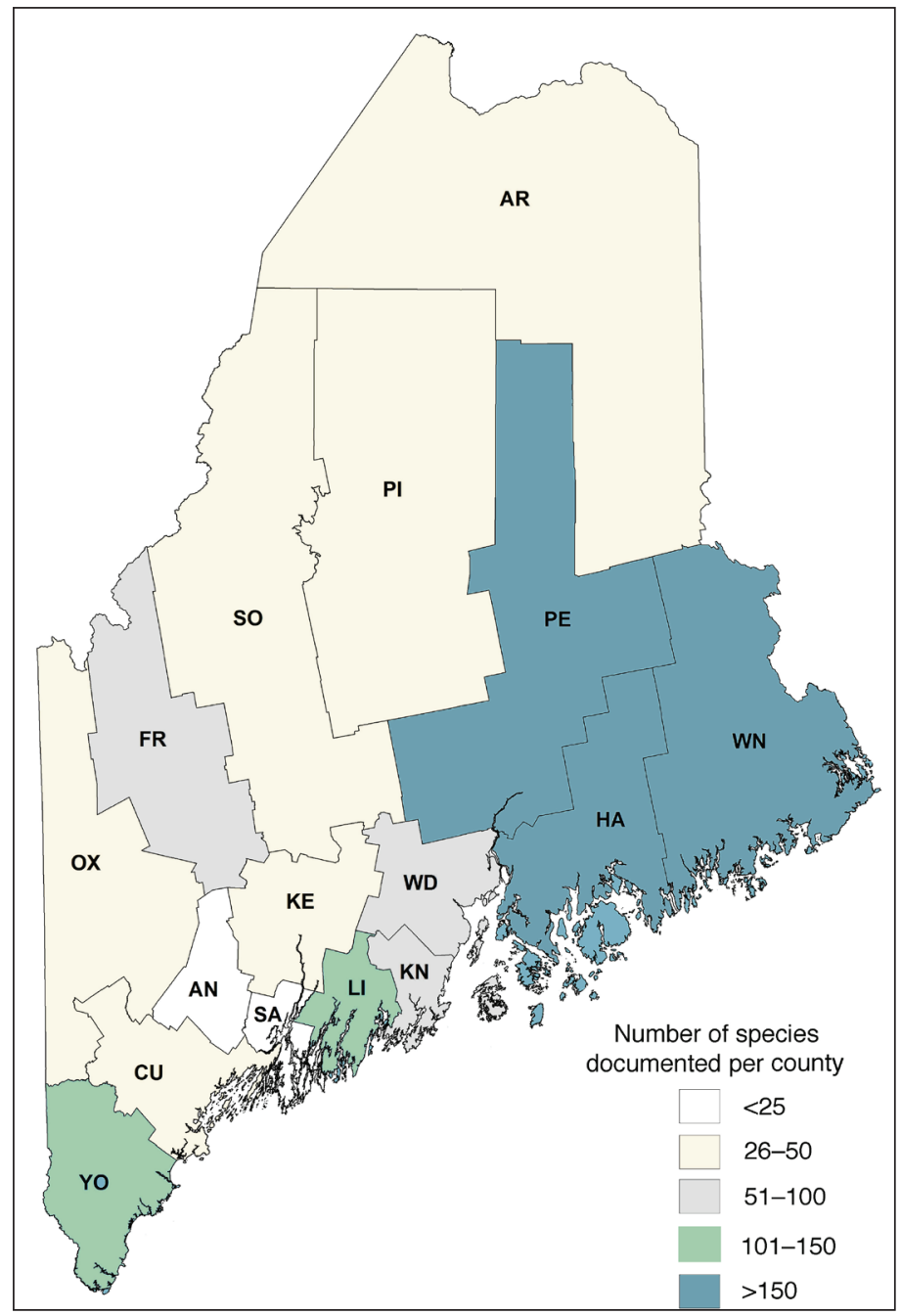


A.C. Dibble, F.A. Drummond, C. Stubbs, M. Veit, and J.S. Ascher

and iDigbio (https://www.idigbio.org/portal/recordsets/8919571f-205a-4aed-b9f2$96 \mathrm{ccd} 0108 \mathrm{e} 4 \mathrm{c}$ ). The citizen-science website Bugguide (www.bugguide.net) was also consulted as a source for recent records for the subset of species identifiable from digital images. The checklist, while preliminary, provides insight into the state of knowledge of bee species for each of Maine's 16 counties (Fig. 1) and provides a baseline for future assessment of native bee community health and diversity in Maine. For example, a 5-year bumble bee survey initiated in 2015 (http:// mainebumblebeeatlas.umf.maine.edu/) will likely expand upon the baseline for bumble bees reported here (Bickerman-Martens et al. 2017).

\section{Methods}

\section{Checklist}

We compiled records for Maine bees from collections we examined (University of Maine Entomological Museum, American Museum of Natural History, Cornell University, Museum of Comparative Zoology, Maine Forest Service Entomological Collection, Acadia National Park, The Peabody Museum of Natural History at Yale University, and several private collections), published bee research conducted in Maine (Boulanger et al. 1967; Bushmann and Drummond 2015; Dibble and Drummond 1997; Dibble et al. 1997; Miliczky 1978; Procter 1938, 1946; Stubbs et al. 1992, 2007), and additional graduate theses and widely disseminated reports (e.g., Dearborn et al. 1983, Droege 2012). We consulted historical literature for Maine records and ranges of bee taxa, including taxonomic revisions by Bouseman and LaBerge (1978), LaBerge (1956, 1961, 1967, 1971, 1973, 1977, 1980, 1985, 1987, 1989), LaBerge and Bouseman (1970), LaBerge and Ribble (1972, 1975), McGinley (1986), Michener (1947), and Ribble (1968). Species distribution information was also derived from recent taxonomic publications such as Gibbs (2010, 2011), Gibbs et al. (2013), Rightmyer et al. (2010), and Sheffield et al. (2011). Published and unpublished databases were consulted including iDigbio; records from specimens integrated by Discover Life from the United States Geological Survey through efforts of S. Droege and displayed using its global mapper tool; the Maine Forest Service Entomological Museum (see Dearborn et al. 1983) with updates (C. Donohue, Maine Forest Service, Augusta, ME, pers. comm.); and the Maine Bumble Bee Atlas (hosted by the Maine Department of Inland Fisheries and Wildlife with the University of Maine). State records for Maine were also obtained from critical review of Mitchell $(1960,1962)$, and from taxonomic revisions that also served as a basis for updating all scientific names to current usage, following Ascher and Pickering (2017) and a pending update of the World Bee Checklist in the Integrated Taxonomic Information System (http://www.itis.gov). New records based on specimens collected since 1992 by A.C. Dibble and from 2009 to 2015 by S. Bushmann, F.A. Drummond, B. DuClos, and $\mathrm{M}$. Veit are also reflected in the checklist.

Many vouchers, especially in older collections, have labels with minimal data regarding localities and collecting events. By contrast, collections made for various research projects in Maine may have voucher specimens with detailed ecological 
A.C. Dibble, F.A. Drummond, C. Stubbs, M. Veit, and J.S. Ascher

and collecting-effort information recorded. Most of these vouchers were obtained in one of the following ways: (1) as an individual bee captured directly on flowers, (2) using sweep nets on flowers of known plants in a repeatable manner, (3) using malaise traps set up at a known plant, (4) using small tent-like nets set up over bee burrows, or (5) using bowl traps with a liquid solution in which insects drown when they arrive to investigate a visual trap mimicking a highly attractive floral resource (Droege 2010). Catch rates for bee groups differ between capture methods (Bushmann and Drummond 2015, Drummond and Stubbs 1997b), but important and complementary data were obtained in Maine by each of the sampling methods. For instance, bowl traps captured large numbers of sweat bees but were ineffective at reliably capturing bumble bees. Hand capture and netting were, by contrast, highly effective for some large and noticeable bees (Bushmann 2013).

The compilation of the checklist by county (Table 2) includes only taxa where historical reports are consistent with species ranges as currently understood and are otherwise considered to be reliable (for some of these, county-level information was unavailable) or for which a specimen is known to us. Additional bee taxa that could be in Maine based on reports that we regard as yet unconfirmed but potentially valid are excluded from the checklist table but are discussed below.

Table 2. Checklist of the bees of Maine by county including selected sources. "Unknown county" means that a locality label or literature source does not include county or town but is from Maine. County abbreviations are explained in Figure 1. Superscripts following county records are representative sources, and are not comprehensive. Numbers refer to sources that are records in the literature or specimens in collections, lower case letters refer mostly to museum specimens (see Source Legend at end of checklist). ${ }^{*}=$ introduced species. [Table continued on the following 10 pages.]

Scientific name

Andrena (Andrena) carolina Viereck, 1909

Andrena (Andrena) clarkella (Kirby, 1802)

Andrena (Andrena) frigida Smith, 1853

Andrena (Andrena) mandibularis Robertson, 1892 Andrena (Andrena) milwaukeensis Graenicher, 1903 Andrena (Andrena) rufosignata Cockerell, 1902

Andrena (Andrena) thaspii Graenicher, 1903

Andrena (Callandrena s.1.) asteris Robertson, 1891 Andrena (Callandrena s.1.) braccata Viereck, 1907 Andrena (Callandrena s.1.) placata Mitchell, 1960 Andrena (Cnemidandrena) canadensis Dalla Torre, 1896 Andrena (Cnemidandrena) hirticincta Provancher, 1888

Andrena (Cnemidandrena) nubecula Smith, 1853

Andrena (Conandrena) bradleyi Viereck, 1907

Andrena (Euandrena) algida Smith, 1853

Andrena (Euandrena) nigrihirta (Ashmead, 1890)
Counties (selected sources)

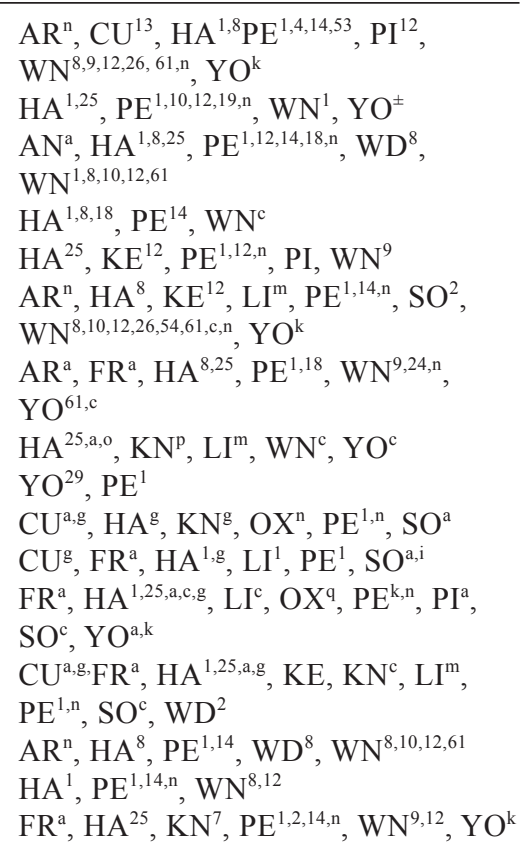


A.C. Dibble, F.A. Drummond, C. Stubbs, M. Veit, and J.S. Ascher

Scientific name

Andrena (Gonandrena) integra Smith, 1853

Andrena (Gonandrena) persimulata Viereck, 1917

Andrena (Holandrena) cressonii Robertson, 1891

Andrena (Larandrena) miserabilis Cresson, 1872

Andrena (Leucandrena) barbilabris (Kirby, 1802)

Andrena (Leucandrena) erythronii Robertson, 1891

Andrena (Melandrena) carlini Cockerell, 1901

Andrena (Melandrena) commoda Smith, 1879

Andrena (Melandrena) dunningi Cockerell, 1898

Andrena (Melandrena) nivalis Smith, 1853

Andrena (Melandrena) pruni Robertson, 1891

Andrena (Melandrena) regularis Malloch, 1917

Andrena (Melandrena) vicina Smith, 1853

Andrena (Micrandrena) melanochroa Cockerell, 1898

Andrena (Micrandrena) salictaria Robertson, 1905

Andrena (Plastandrena) crataegi Robertson, 1893

Andrena (Rhacandrena) brevipalpis Cockerell, 1930

Andrena (Rhacandrena) robertsonii Dalla Torre, 1896

Andrena (Scrapteropsis) alleghaniensis Viereck, 1907

Andrena (Scrapteropsis) imitatrix Cresson, 1872

Andrena (Scrapteropsis) kalmiae Atwood, 1934

Andrena (Simandrena) nasonii Robertson, 1895

Andrena (Simandrena) wheeleri Graenicher, 1904

*Andrena (Taeniandrena) wilkella (Kirby, 1802)

Andrena (Thysandrena) bisalicis Viereck, 1908

Andrena (Thysandrena) w-scripta Viereck, 1904

Andrena (Trachandrena) ceanothi Viereck, 1917

Andrena (Trachandrena) forbesii Robertson, 1891

Andrena (Trachandrena) hippotes Robertson, 1895

Andrena (Trachandrena) miranda Smith, 1879

Andrena (Trachandrena) nuda Robertson, 1891

Andrena (Trachandrena) rugosa Robertson, 1891

Andrena (Trachandrena) sigmundi Cockerell, 1902

Andrena (Trachandrena) spiraeana Robertson, 1895

Andrena (Trachandrena) virginiana Mitchell, 1960

Andrena (Tylandrena) erythrogaster (Ashmead, 1890)

Andrena (Tylandrena) perplexa Smith, 1853

Calliopsis (Calliopsis) andreniformis Smith, 1853

Perdita (Perdita) octomaculata (Say, 1824)

Pseudopanurgus aestivalis (Provancher, 1882) $(=P$.

nebraskensis)
Counties (selected sources)

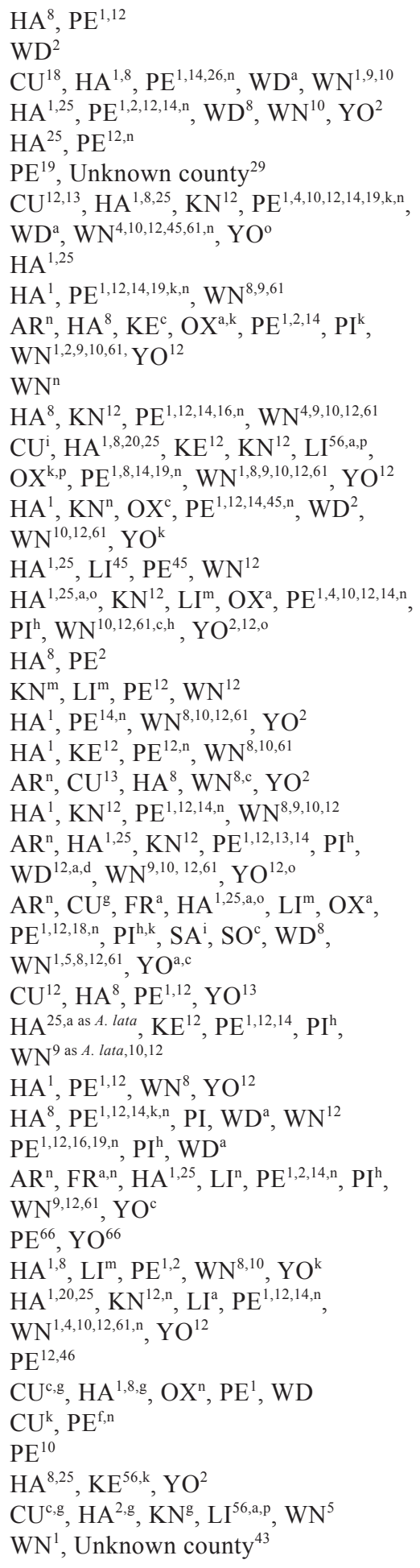


A.C. Dibble, F.A. Drummond, C. Stubbs, M. Veit, and J.S. Ascher

Scientific name

Pseudopanurgus andrenoides (Smith, 1853)

Anthophora (Clisodon) terminalis Cresson, 1869

Anthophora (Melea) bomboides Kirby, 1837

*Apis (Apis) mellifera L., 1758

Bombus (Bombus) affinis Cresson, 1863

Bombus (Bombus) terricola Kirby, 1837

Bombus (Cullumanobombus) griseocollis (DeGeer, 1773)

Bombus (Cullumanobombus) rufocinctus Cresson, 1863

Bombus (Thoracobombus) fervidus (Fabricius, 1798)

Bombus (Thoracobombus) pensylvanicus (DeGeer, 1773)

Bombus (Psithyrus) ashtoni (Cresson, 1864)

Bombus (Psithyrus) citrinus (Smith, 1854)

Bombus (Psithyrus) fernaldae (Franklin, 1911)

Bombus (Psithyrus) insularis (Smith 1861)

Bombus (Pyrobombus) bimaculatus Cresson, 1863

Bombus (Pyrobombus) impatiens Cresson, 1863

Bombus (Pyrobombus) perplexus Cresson, 1863

Bombus (Pyrobombus) sandersoni Franklin, 1913

Bombus (Pyrobombus) ternarius Say, 1837

Bombus (Pyrobombus) vagans vagans Smith, 1854

Bombus (Subterraneobombus) borealis Kirby, 1837

Habropoda laboriosa (Fabricius, 1804)

Melissodes (Apomelissodes) apicatus Lovell \& Cockerell, 1906

Melissodes (Eumelissodes) agilis Cresson, 1878

Melissodes (Heliomelissodes) desponsus Smith, 1854

Melissodes (Eumelissodes) druriellus (Kirby, 1802)

Melissodes (Eumelissodes) illatus Lovell \& Cockerell, 1906
Counties (selected sources)

$\mathrm{FR}^{57, \mathrm{a}}, \mathrm{HA}^{1,2, \mathrm{c}}, \mathrm{KN}^{\mathrm{c}}, \mathrm{LI}^{56}, \mathrm{PE}^{1}, \mathrm{SO}^{\mathrm{a}}$

$\mathrm{HA}^{25}, \mathrm{OX}^{\mathrm{q}}, \mathrm{PE}^{1}, \mathrm{SO}^{2}, \mathrm{YO}^{\mathrm{k}}$

Unknown county ${ }^{28}$

$\mathrm{AR}^{4}, \mathrm{AN}^{4}, \mathrm{CU}^{13}, \mathrm{HA}^{1,4,25,63}, \mathrm{KE}^{4}$

$\mathrm{KN}^{4,70}, \mathrm{LI}^{4,70}, \mathrm{OX}^{4, \mathrm{n}}, \mathrm{PE}^{1,4,12,69,63,70, \mathrm{k}}$,

$\mathrm{WD}^{4,8,63,70, \mathrm{q}}, \mathrm{WN}^{1,4,5,9,10,61,63,70}$

$\mathrm{CU}^{\mathrm{g}, \mathrm{i}}, \mathrm{FR}^{27}, \mathrm{HA}^{25}, \mathrm{KE}^{\mathrm{k}}, \mathrm{LI}^{\mathrm{h}, \mathrm{m}}, \mathrm{PE}^{12, \mathrm{n}, \mathrm{p}}$,

$\mathrm{PI}^{\mathrm{a}}, \mathrm{WD}^{4,12}, \mathrm{WN}^{12}, \mathrm{YO}^{\mathrm{a}}$

$\mathrm{AR}^{1,4,8, \mathrm{p}}, \mathrm{CU}^{4, \mathrm{a}, \mathrm{g}, \mathrm{o}}, \mathrm{FR}^{1,4,27,57,63, \mathrm{a}, \mathrm{o}}$,

$\mathrm{HA}^{1,4,18,25,63,70, \mathrm{a}, \mathrm{f}, \mathrm{g}, \mathrm{q}}, \mathrm{KE}^{4, \mathrm{k}}, \mathrm{KN}^{4,70, \mathrm{a}, \mathrm{g}}, \mathrm{LI}^{\mathrm{o}}$,

$\mathrm{OX}^{\mathrm{a}}, \mathrm{PE}^{1,4,12,13,14,18,63,69,70, \mathrm{k}}, \mathrm{PI}^{\mathrm{a}, \mathrm{h}, \mathrm{k}}, \mathrm{SA}^{4}$,

$\mathrm{SO}^{4, \mathrm{i}}, \mathrm{WD}^{4,63,70, \mathrm{p}, \mathrm{q}}, \mathrm{WN}^{1,4,5,9,12,61,63,70}$,

$\mathrm{YO}^{4,73, \mathrm{a}, \mathrm{k}, \mathrm{r}}$

$\mathrm{HA}^{1,63}, \mathrm{PE}^{1,63}, \mathrm{PI}^{\mathrm{a}}, \mathrm{WN}^{\mathrm{b}}$

$\mathrm{AR}^{\mathrm{j}, \mathrm{n}}, \mathrm{HA}^{1}, \mathrm{PE}^{1,63, \mathrm{~b}}, \mathrm{WN}^{5}$

$\mathrm{HA}^{1,25, \mathrm{~m}}, \mathrm{KE}^{\mathrm{k}, \mathrm{n}}, \mathrm{LI}^{\mathrm{p}}, \mathrm{PE}^{1,12}, \mathrm{OX}^{\mathrm{q}}, \mathrm{PI}^{\mathrm{a}}$,

$\mathrm{SO}^{2}, \mathrm{WN}^{4,61}, \mathrm{YO}^{\mathrm{a}, \mathrm{r}}$

$\mathrm{YO}^{29,32,66}$

$\mathrm{CU}^{\mathrm{a}, \mathrm{n}}, \mathrm{FR}^{57, \mathrm{a}}, \mathrm{HA}^{1,25, \mathrm{k}, \mathrm{o}, \mathrm{q}}, \mathrm{KE}^{\mathrm{k}, \mathrm{n}}, \mathrm{LI}^{\mathrm{o}}$,

$\mathrm{OX}^{\mathrm{a}}, \mathrm{PE}^{1,2, \mathrm{n}}, \mathrm{YO}^{\mathrm{a}, \mathrm{r}}$

$\mathrm{AR}^{8}, \mathrm{CU}^{\mathrm{r}}, \mathrm{HA}^{25,63, \mathrm{k}, \mathrm{p}}, \mathrm{KE}^{\mathrm{k}}, \mathrm{KN}^{\mathrm{a}}, \mathrm{LI}^{\mathrm{p}}$,

$\mathrm{OX}^{\mathrm{k}}, \mathrm{PE}^{\mathrm{p}}, \mathrm{WD}^{\mathrm{p}}, \mathrm{WN}^{\mathrm{c}}, \mathrm{YO}^{73}$

$\mathrm{FR}^{57, \mathrm{a}}, \mathrm{HA}^{2,25, \mathrm{p}}, \mathrm{PE}^{1}, \mathrm{PI}^{12}, \mathrm{WD}^{63}$ $\mathrm{WN}^{1,4,5,63}$

$\mathrm{FR}^{\mathrm{a}}, \mathrm{HA}^{\mathrm{n}}, \mathrm{PE}^{1}$

$\mathrm{AR}^{8}, \mathrm{HA}^{1,25,63}, \mathrm{KE}^{2}, \mathrm{KN}^{63}, \mathrm{LI}^{6, \mathrm{j}}$, $\mathrm{PE}^{1,2,63, \mathrm{~b}}, \mathrm{WD}^{5,63}, \mathrm{WN}^{1,4,61,63}, \mathrm{YO}^{2}$

$\mathrm{AR}^{8}, \mathrm{CU}^{1,13}, \mathrm{FR}^{4,8}, \mathrm{HA}^{1,4,8,63, \mathrm{~m}}, \mathrm{KE}^{4, \mathrm{k}, \mathrm{n}}$, $\mathrm{KN}^{4,63, \mathrm{~g}}, \mathrm{LI}^{4}, \mathrm{OX}^{\mathrm{n}}, \mathrm{PE}^{1,2,4,12,63, \mathrm{~b}}, \mathrm{PI}^{\mathrm{a}, \mathrm{m}}$,

$\mathrm{SA}^{\mathrm{i}}, \mathrm{WD}^{4,63}, \mathrm{WN}^{1,4,61,63}, \mathrm{YO}^{1,73, \mathrm{a}}$

$\mathrm{AR}^{2,8}, \mathrm{HA}^{1,2,25,63, \mathrm{a}, \mathrm{j}, \mathrm{q}}, \mathrm{KE}^{\mathrm{k}}, \mathrm{LI}^{\mathrm{m}}, \mathrm{KN}^{63, \mathrm{a}}$,

$\mathrm{PE}^{1,2,12,14,63, \mathrm{~m}}, \mathrm{PI}^{\mathrm{h}, \mathrm{k}}, \mathrm{WD}^{63, \mathrm{q}}, \mathrm{WN}^{1,5,12,63, \mathrm{~b}}$, $\mathrm{YO}^{\mathrm{r}}$

$\mathrm{AN}^{\mathrm{b}}, \mathrm{AR}, \mathrm{FR}^{\mathrm{a}}, \mathrm{HA}^{63, \mathrm{a}}, \mathrm{KE}^{\mathrm{b}}, \mathrm{KN}^{\mathrm{a}}, \mathrm{PE}^{1,63}$, $\mathrm{PI}^{\mathrm{k}, \mathrm{m}}, \mathrm{WN}^{1,4,5,63, \mathrm{~b}}, \mathrm{YO}^{\mathrm{q}}$

$\mathrm{AR}^{1,2,8}, \mathrm{CU}^{\mathrm{a}, \mathrm{g}, \mathrm{r}}, \mathrm{FR}^{4,57, \mathrm{a}, \mathrm{o}}$,

$\mathrm{HA}^{1,4,25,63, \mathrm{a}, \mathrm{g}, \mathrm{k}, \mathrm{m}, \mathrm{n}, \mathrm{p}, \mathrm{q}}, \mathrm{KE}^{4, \mathrm{~m}, \mathrm{n}}, \mathrm{KN}^{4,63 \mathrm{a}}$, $\mathrm{LI}^{\mathrm{j}, \mathrm{o}, \mathrm{p}, \mathrm{q}}, \mathrm{OX}^{\mathrm{n}}, \mathrm{PE}^{1,4,12,18,63, \mathrm{k}}, \mathrm{PI}^{\mathrm{a}, \mathrm{h}, \mathrm{k}}, \mathrm{SA}^{4}$,

$\mathrm{SO}^{\mathrm{a}, \mathrm{j}}, \mathrm{WD}^{4,63, \mathrm{k}, \mathrm{p}, \mathrm{q}}, \mathrm{WN}^{1,4,5,9,61,63, \mathrm{a}, \mathrm{b}}, \mathrm{YO}^{\mathrm{a}, \mathrm{r}}$

$\mathrm{AR}^{1,4}, \mathrm{CU}^{13, \mathrm{~g}, \mathrm{p}, \mathrm{r}}, \mathrm{FR}^{4,57, \mathrm{a}}, \mathrm{HA}^{1,19,25,63, \mathrm{a}, \mathrm{k}, \mathrm{q}}$,

$\mathrm{KN}^{63, \mathrm{a}}, \mathrm{LI}^{\mathrm{m}}, \mathrm{PE}^{1,2,10,63, \mathrm{~m}, \mathrm{n}}, \mathrm{PI}^{\mathrm{a}, \mathrm{h}}, \mathrm{SO}^{2}$,

$\mathrm{WD}^{63, \mathrm{k}, \mathrm{q}}, \mathrm{WN}^{1,4,5,9,10,61,63}, \mathrm{YO}^{\mathrm{a}}$

$\mathrm{AR}^{1,2,4,8}, \mathrm{FR}^{4,57, \mathrm{a}}, \mathrm{HA}^{1,2,25}, \mathrm{KN}^{63, \mathrm{e}}, \mathrm{LI}^{\mathrm{p}}$,

$\mathrm{OX}^{\mathrm{q}}, \mathrm{PE}^{1,63, \mathrm{~b}}, \mathrm{WD}^{5,8,63, \mathrm{q}} \mathrm{WN}^{1,4}$

$\mathrm{LI}^{\mathrm{m}}$

$\mathrm{LI}^{48, \mathrm{~m}, \mathrm{p}}, \mathrm{WD}^{48}$

$\mathrm{LI}^{47}$,a

$\mathrm{LI}^{\mathrm{a}}, \mathrm{PE}^{1}$

$\mathrm{AN}^{47, \mathrm{o}}, \mathrm{AR}^{47}, \mathrm{CU}^{47, \mathrm{c}}, \mathrm{HA}^{1,25, \mathrm{a}}, \mathrm{LI}^{47}$, $\mathrm{PE}^{1,47}$

$\mathrm{FR}^{\mathrm{a}, \mathrm{o}}, \mathrm{HA}^{1,2,8,25,47, \mathrm{a}}, \mathrm{KE}^{47}, \mathrm{KN}^{\mathrm{a}}, \mathrm{LI}^{47}$, $\mathrm{PE}^{1, \mathrm{n}}, \mathrm{SO}^{\mathrm{a}}, \mathrm{WN}^{1,4,5,7,12, \mathrm{~b}, \mathrm{c}}, \mathrm{YO}^{47, \mathrm{o}}$ 
A.C. Dibble, F.A. Drummond, C. Stubbs, M. Veit, and J.S. Ascher

Scientific name

Melissodes (Eumelissodes) subillatus LaBerge, 1961

Melissodes (Eumelissodes) trinodis Robertson, 1901

Melissodes (Melissodes) b. bimaculatus (Lepeletier, 1825)

Peponapis (Peponapis) pruinosa (Say, 1837)

Holcopasites calliopsidis (Linsley, 1943)

Holcopasites illinoiensis (Robertson, 1891)

Epeolus americanus Cresson, 1878 [= lanhami Mitchell, 1962]

Epeolus autumnalis Robertson, 1902

Epeolus pusillus Cresson, 1864

Epeolus scutellaris Say, 1824

Epeoloides pilosulus (Cresson, 1878)

Triepeolus donatus (Smith, 1854)

Triepeolus pectoralis (Robertson, 1897)

Nomada armatella Cockerell, 1903

Nomada articulata Smith, 1854

Nomada bella Cresson, 1863

Nomada bethunei Cockerell, 1903

Nomada composita Mitchell, 1962

Nomada cressonii Robertson, 1893

Nomada cuneata (Robertson, 1903)

Nomada denticulata Robertson, 1902

Nomada depressa Cresson, 1863

Nomada dreisbachi Mitchell, 1962

Nomada florilega Lovell and Cockerell, 1905

Nomada gracilis Cresson, 1863

Nomada illinoensis Robertson, 1900

Nomada imbricata Smith, 1854

Nomada lepida Cresson, 1863

Nomada louisianae Cockerell, 1903

Nomada luteoloides Robertson, 1895

Nomada maculata Cresson, 1863

Nomada ovata (Robertson, 1903)

Nomada perplexa Cresson, 1863

Nomada proxima Cresson, 1863

Nomada pygmaea Cresson, 1863

Nomada sayi Robertson, 1893

Nomada subrutila Lovell \& Cockerell, 1905

Nomada valida Smith, 1854

Nomada vicina Cresson, 1863

Nomada vincta Say, 1837

Nomada xanthura Cockerell, 1908

Ceratina (Zadontomerus) calcarata Robertson, 1900

Ceratina (Zadontomerus) dupla Say, 1837

Ceratina (Zadontomerus) mikmaqi Rehan \& Sheffield, 2011

Xylocopa (Xylocopoides) virginica (L., 1771)

Colletes americanus Cresson, 1868

Colletes compactus Cresson, 1868

Colletes consors Cresson, 1868 [ssp. mescocopus Swenk]
Counties (selected sources)

$\mathrm{CU}^{47}, \mathrm{HA}^{47}, \mathrm{LI}^{47, \mathrm{~m}}$

$\mathrm{LI}^{62, \mathrm{a}}$

Unknown county ${ }^{66}$

$\mathrm{CU}^{1}, \mathrm{HA}^{1}, \mathrm{PE}^{4}$

$\mathrm{WA}^{1}$

$\mathrm{PE}^{41}$

$\mathrm{WN}^{40, \mathrm{c}}$

$\mathrm{HA}^{1}$

Unknown county ${ }^{66}$

$\mathrm{CU}^{6, \mathrm{a}}, \mathrm{HA}^{1,25, \mathrm{a}, \mathrm{g}}, \mathrm{KN}^{\mathrm{a}, \mathrm{g}}, \mathrm{WN}^{1}$

$\mathrm{LI}^{\mathrm{m}} \mathrm{YO}^{2}$

$\mathrm{HA}^{25}, \mathrm{LI}^{56, \mathrm{a}, \mathrm{m}}$

$\mathrm{CU}^{\mathrm{g}}, \mathrm{HA}^{\mathrm{c}}, \mathrm{KN}^{\mathrm{a}}$

$\mathrm{WN}^{66}$

$\mathrm{CU}^{\mathrm{n}}, \mathrm{HA}^{8}, \mathrm{LI}^{\mathrm{m}}, \mathrm{PE}^{14}, \mathrm{PI}^{\mathrm{k}}$

$\mathrm{PE}^{14,16}, \mathrm{WD}^{2}$

$\mathrm{HA}^{8}, \mathrm{WN}^{8}$

$\mathrm{HA}^{\mathrm{a}}, \mathrm{PE}^{14}, \mathrm{WN}^{10,45}$

$\mathrm{AN}^{\mathrm{m}}, \mathrm{HA}^{1,25}, \mathrm{PE}^{1,14,16}, \mathrm{WD}^{2}, \mathrm{WN}^{9,10,45}$,

$\mathrm{YO}^{2}$

$\mathrm{HA}^{25}$

$\mathrm{PE}^{18}, \mathrm{YO}^{2}$

$\mathrm{OX}^{\mathrm{q}}, \mathrm{PE}^{14}, \mathrm{WN}^{8,9}$

Unknown county ${ }^{29}$

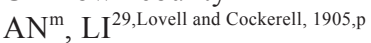

$\mathrm{HA}^{\text {as } N \text {. inepta }}, \mathrm{PE}^{1}, \mathrm{WN}^{8,10}$

Unknown county ${ }^{58}$

$\mathrm{HA}^{8}, \mathrm{PE}^{1,14}, \mathrm{WN}^{8,10}$

$\mathrm{PE}^{14}, \mathrm{WD}^{2}$

Unknown county $^{71}$

$\mathrm{HA}^{1}, \mathrm{PE}^{14}, \mathrm{WD}^{8}, \mathrm{WN}^{1,10,45}$

$\mathrm{HA}^{8}, \mathrm{PE}^{14}, \mathrm{WN}^{8}, \mathrm{YO}^{2}$

$\mathrm{PE}^{14,17,29}$

$\mathrm{HA}^{25}, \mathrm{LI}^{\mathrm{m}}, \mathrm{PE}^{14}$

Unknown county 28, Cresson 1863

$\mathrm{HA}^{5}, \mathrm{PE}^{1,2,14}, \mathrm{WN}^{9,10}$

$\mathrm{HA}^{1}, \mathrm{PE}^{14}, \mathrm{WN}^{9,10}$

$\mathrm{LI}^{\text {Lovell and Cockerell, 1905,p }}$

$\mathrm{HA}^{8}, \mathrm{WN}^{10}$

$\mathrm{HA}^{\mathrm{g}}$

$\mathrm{PE}^{\mathrm{n}}$

Unknown county ${ }^{29}$

$\mathrm{HA}^{1,2,25, \mathrm{o}}, \mathrm{LI}^{\mathrm{m}}, \mathrm{PE}^{1,2,12,14}, \mathrm{SA}^{\mathrm{i}}, \mathrm{WD}^{2}$,

$\mathrm{WN}^{1,8,61}, \mathrm{YO}^{2}$

$\mathrm{HA}^{1,25,0}, \mathrm{KN}^{12}, \mathrm{LI}^{\mathrm{m}}, \mathrm{PE}^{1,10,12,14, \mathrm{n}}, \mathrm{SA}^{\mathrm{i}}$,

$\mathrm{WN}^{9}$

$\mathrm{HA}^{1,8}, \mathrm{SO}^{\mathrm{a}}, \mathrm{WD}^{\mathrm{a}}, \mathrm{WN}^{8}$

$\mathrm{HA}^{1}$

$\mathrm{WN}^{1}$

$\mathrm{HA}^{1}, \mathrm{PE}^{18}, \mathrm{YO}^{\mathrm{a}}$

$\mathrm{HA}^{8,25, \mathrm{a}}, \mathrm{LI}^{\mathrm{a}}, \mathrm{WN}^{12,17}$ 
A.C. Dibble, F.A. Drummond, C. Stubbs, M. Veit, and J.S. Ascher

Scientific name

Counties (selected sources)

Colletes hyalinus Provancher, 1888

$\mathrm{FR}^{\mathrm{a}}$

Colletes impunctatus Nylander, 1852 [ssp. lacustris Swenk]

Colletes inaequalis Say, 1837

Colletes kincaidii Cockerell, 1898

Colletes latitarsis Robertson, 1891

Colletes simulans Cresson, 1868

Colletes validus Cresson, 1868

Hylaeus (Hylaeus) annulatus (L., 1758)

Hylaeus (Cephalylaeus) basalis (Smith, 1853)

Hylaeus (Hylaeus) mesillae (Cockerell, 1896) [ssp. cressoni

(Cockerell, 1907)]

Hylaeus (Hylaeus) saniculae (Robertson, 1896)

Hylaeus (Hylaeus) verticalis (Cresson, 1869)

Hylaeus (Metziella) sparsus (Cresson, 1869)

Hylaeus (Paraprosopis) floridanus (Robertson, 1893)

Hylaeus (Prosopis) affinis (Smith, 1853)

Hylaeus (Prosopis) illinoisensis (Robertson, 1896)

Hylaeus (Prosopis) modestus modestus Say, 1837

Dufourea novaeangliae (Robertson, 1897)

Augochlora (Augochlora) pura (Say, 1837)

Augochlorella aurata (Smith, 1853)

Augochloropsis (Paraugochloropsis) metallica (Fabricius, 1793)

Agapostemon (Agapostemon) sericeus (Förster, 1771)

$\mathrm{PI}^{29,39}$

$\mathrm{HA}^{1,8}, \mathrm{KE}^{\mathrm{k}}, \mathrm{PE}^{1,12,16,18,19}, \mathrm{WN}^{8,10,12,61, \mathrm{n}}$

$\mathrm{SO}^{\mathrm{i}}, \mathrm{WN}^{1}$

Unknown county ${ }^{72}$

$\mathrm{HA}^{2,25}, \mathrm{KN}^{\mathrm{c}}, \mathrm{LI}^{1, \mathrm{a}}, \mathrm{PE}^{1, \mathrm{n}}, \mathrm{SO}^{\mathrm{a}}, \mathrm{WN}^{1,5,12}$

$\mathrm{YO}^{12}$, Unknown county ${ }^{29}$

$\mathrm{AR}^{4}, \mathrm{FR}^{\mathrm{a}, \mathrm{s}}, \mathrm{HA}^{2,4,25, \mathrm{~s}}, \mathrm{KN}^{\mathrm{a}}, \mathrm{PE}^{1,4,12}, \mathrm{P}^{4}$,

$\mathrm{WD}^{4}, \mathrm{WN}^{1,4,5,9}$

$\mathrm{FR}^{\mathrm{a}}, \mathrm{LI}^{38}, \mathrm{WN}^{9}$

$\mathrm{CU}^{\mathrm{a}}, \mathrm{HA}^{1,25, \mathrm{a}}, \mathrm{LI}^{38, \mathrm{~m}}, \mathrm{PE}^{1}, \mathrm{SO}^{\mathrm{a}}, \mathrm{WN}^{1,8}$,

$\mathrm{YO}^{2}$

$\mathrm{LI}^{\mathrm{a}}$

$\mathrm{HA}^{8}, \mathrm{LI}^{\mathrm{m}}, \mathrm{PE}^{1}, \mathrm{PI}, \mathrm{WN}^{9}$

$\mathrm{HA}^{25}$

Unknown county ${ }^{33}$

$\mathrm{CU}^{\mathrm{a}}, \mathrm{FR}^{\mathrm{a}}, \mathrm{HA}^{1,2,8,25, \mathrm{a}}, \mathrm{KN}, \mathrm{LI}^{\mathrm{a}}, \mathrm{PE}^{1,2,12,0}$, $\mathrm{WN}^{8}$

Unknown county ${ }^{29}$

$\mathrm{FR}^{57}, \mathrm{HA}^{1,25}, \mathrm{PE}^{1,2,12,14}, \mathrm{PI}, \mathrm{WN}^{1,9}$

$\mathrm{HA}^{25}$, LI, $\mathrm{WN}^{42}$

$\mathrm{FR}^{\mathrm{c}}, \mathrm{HA}^{1,8,25}, \mathrm{KN}^{\mathrm{m}}, \mathrm{PE}^{1}, \mathrm{WD}^{\mathrm{k}}, \mathrm{WN}^{9,61}$

$\mathrm{CU}^{\mathrm{a}}, \mathrm{HA}^{1,25, \mathrm{a}}, \mathrm{KE}^{\mathrm{n}}, \mathrm{LI}^{\mathrm{a}, \mathrm{m}}, \mathrm{OX}^{\mathrm{q}}, \mathrm{PE}^{1,12,14, \mathrm{n}}$, $\mathrm{SO}^{\mathrm{a}}, \mathrm{WD}^{8,12, \mathrm{a}}, \mathrm{WN}^{1,4,5,9,10,12,61}, \mathrm{YO}^{2, \mathrm{f}, \mathrm{k}}$

$\mathrm{WD}^{8}$

$\mathrm{OX}^{\mathrm{q}}$

Agapostemon (Agapostemon) splendens (Lepeletier de Saint $\mathrm{PE}^{\mathrm{n}}, \mathrm{SA}^{\mathrm{i}}$

Fargeau, 1841)

Agapostemon (Agapostemon) texanus Cresson, 1872

Agapostemon (Agapostemon) virescens (Fabricius, 1775)

Halictus (Odontalictus) ligatus Say, 1837

Halictus (Seladonia) confusus confusus Smith, 1853

Halictus (Protohalictus) rubicundus (Christ, 1791)

Lasioglossum (Dialictus) admirandum (Sandhouse, 1924)

Lasioglossum (Dialictus) albipenne (Robertson, 1890)

Lasioglossum (Dialictus) anomalum (Robertson, 1892)

Lasioglossum (Dialictus) atwoodi Gibbs, 2010

Lasioglossum (Dialictus) coeruleum (Robertson, 1893)

Lasioglossum (Dialictus) cressonii (Robertson, 1890)

Lasioglossum (Dialictus) ellisiae (Sandhouse, 1924)

Lasioglossum (Dialictus) ephialtum Gibbs, 2010

Lasioglossum (Dialictus) heterognathum (Mitchell, 1960)

Lasioglossum (Dialictus) hitchensi Gibbs, 2012

Lasioglossum (Dialictus) imitatum (Smith, 1853)

Lasioglossum (Dialictus) katherineae (Gibbs, 2011)

Lasioglossum (Dialictus) laevissimum (Smith, 1853)

Lasioglossum (Dialictus) leucocomum (Lovell, 1908)

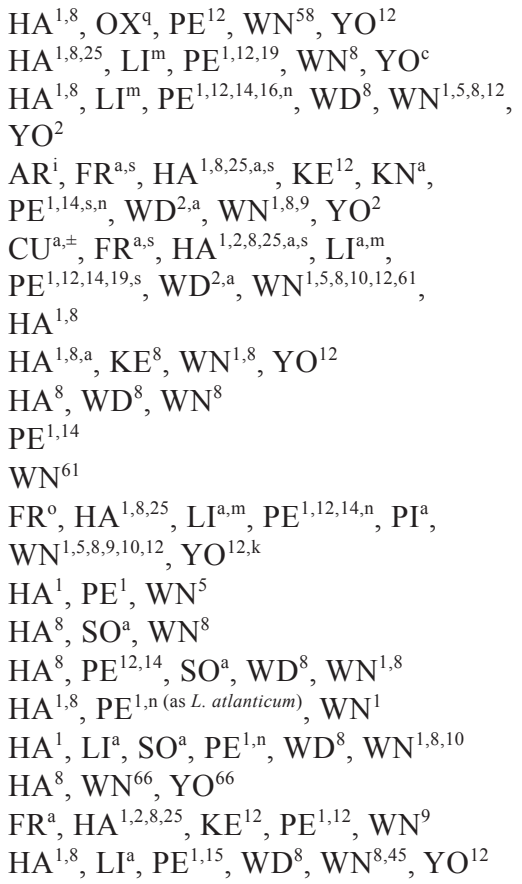


A.C. Dibble, F.A. Drummond, C. Stubbs, M. Veit, and J.S. Ascher

Scientific name

Lasioglossum (Dialictus) lineatulum (Crawford, 1906)

Lasioglossum (Dialictus) nigroviride (Graenicher, 1911)

Lasioglossum (Dialictus) novascotiae (Mitchell, 1960)

Lasioglossum (Dialictus) oblongum (Lovell, 1905)

Lasioglossum (Dialictus) oceanicum (Cockerell, 1916)

Lasioglossum (Dialictus) obscurum (Robertson, 1892)

Lasioglossum (Dialictus) paradmirandum (Knerer \&

Atwood, 1966)

Lasioglossum (Dialictus) perpunctatum (Ellis, 1913)

Lasioglossum (Dialictus) pilosum (Smith, 1853)

Lasioglossum (Dialictus) planatum (Lovell, 1905)

Lasioglossum (Dialictus) smilacinae (Roberson, 1899)

Lasioglossum (Dialictus) subversans (Mitchell, 1960)

Lasioglossum (Dialictus) subviridatum (Cockerell, 1938)

Lasioglossum (Dialictus) taylorae (Gibbs, 2010)

Lasioglossum (Dialictus) tenax (Sandhouse, 1924)

Lasioglossum (Dialictus) timothyi (Gibbs, 2010)

Lasioglossum (Dialictus) versans (Lovell, 1905)

Lasioglossum (Dialictus) versatum (Robertson, 1902)

Lasioglossum (Dialictus) viridatum (Lovell, 1905)

Lasioglossum (Dialictus) weemsi (Mitchell, 1960)

Lasioglossum (Dialictus) zephyrum (Smith, 1853)

Lasioglossum (Evylaeus) cinctipes (Provancher, 1888)

Lasioglossum (Hemihalictus) birkmanni (Crawford, 1906)

(former L. macoupinense sensu auct.)

Lasioglossum (Hemihalictus) foxii (Robertson, 1895)

Lasioglossum (Hemihalictus) inconditum (Cockerell, 1916)

Lasioglossum (Hemihalictus) macoupinense (Robertson,

1895) [non auct.; = divergens (Lovell, 1905)]

Lasioglossum (Hemihalictus) nelumbonis (Robertson, 1890)

Lasioglossum (Hemihalictus) pectorale (Smith, 1853)

Lasioglossum (Lasioglossum) acuminatum McGinley, 1986

Lasioglossum (Lasioglossum) athabascense (Sandhouse, 1933)

Lasioglossum (Lasioglossum) coriaceum (Smith, 1853)

*Lasioglossum (Lasioglossum) zonulum (Smith, 1848)

*Lasioglossum (Leuchalictus) leucozonium (Schrank, 1781)

Lasioglossum (Sphecodogastra) comagenense (Knerer \&

Atwood, 1964)

Lasioglossum (Sphecodogastra) quebecense (Crawford, 1907)

Lasioglossum (Sphecodogastra) truncatum (Robertson, 1901)

Sphecodes atlantis Mitchell, 1956

Sphecodes clematidis Robertson, 1897

Sphecodes confertus Say, 1837

Sphecodes coronus Mitchell, 1956
Counties (selected sources)

$\mathrm{PE}^{\mathrm{n}}, \mathrm{WD}, \mathrm{WN}^{8}, \mathrm{YO}^{\mathrm{q}}$

$\mathrm{FR}^{\mathrm{a}}, \mathrm{HA}^{1,8}, \mathrm{KN}^{\mathrm{a}}, \mathrm{LI}^{\mathrm{m}}, \mathrm{PE}^{1,12, \mathrm{n}}$

$\mathrm{KN}^{\mathrm{a}}$

$\mathrm{HA}^{1,25,0}, \mathrm{LI}^{\mathrm{m}, \mathrm{o}}, \mathrm{PE}^{1,14}, \mathrm{WN}^{1}$

$\mathrm{HA}^{25}, \mathrm{WN}^{8}$

$\mathrm{LI}^{\mathrm{m}}, \mathrm{PE}^{\mathrm{m}}$

$\mathrm{HA}^{8}, \mathrm{WN}^{8}$

$\mathrm{HA}^{1,8}, \mathrm{PE}^{1}, \mathrm{WN}^{1,8}, \mathrm{YO}^{12}$

$\mathrm{HA}^{1,2}, \mathrm{YO}^{12}$

$\mathrm{FR}^{\mathrm{a}}, \mathrm{HA}^{1,2,8}, \mathrm{LI}^{\mathrm{a}}, \mathrm{PE}^{1}, \mathrm{WD}^{8}, \mathrm{YO}^{2}$

$\mathrm{HA}^{1,8}, \mathrm{SO}^{\mathrm{c}}, \mathrm{PE}^{12,19}, \mathrm{WD}^{8}, \mathrm{WN}^{8}, \mathrm{YO}^{12}$

$\mathrm{FR}^{\mathrm{a}}, \mathrm{HA}^{2,8}, \mathrm{WD}^{8}$

$\mathrm{HA}^{8}, \mathrm{WN}^{8}$

$\mathrm{HA}^{8}, \mathrm{PE}^{1}$

$\mathrm{FR}^{\mathrm{a}}, \mathrm{HA}^{2}, \mathrm{WN}^{9}$

$\mathrm{HA}^{8}, \mathrm{WN}^{8,10}, \mathrm{YO}^{24}$

$\mathrm{HA}^{1,2,8, \mathrm{a}}, \mathrm{LI}^{\mathrm{a}}, \mathrm{PE}^{1, \mathrm{n}}, \mathrm{WD}^{\mathrm{a}}, \mathrm{WN}^{8,9}$

$\mathrm{HA}^{1,2,8,25}, \mathrm{PE}^{1, \mathrm{n}}, \mathrm{SO}^{\mathrm{a}}, \mathrm{WN}^{8}$

$\mathrm{FR}^{\mathrm{a}}, \mathrm{HA}^{1,25, \mathrm{a}}, \mathrm{KE}, \mathrm{LI}^{\mathrm{m}}, \mathrm{PE}^{1, \mathrm{~m}}, \mathrm{WD}^{8}$, $\mathrm{WN}^{1,9}$

$\mathrm{HA}^{8}, \mathrm{WD}^{8}, \mathrm{WN}^{8}$

$\mathrm{HA}^{25}$

$\mathrm{HA}^{8,25, \mathrm{c}}, \mathrm{KE}^{2, \mathrm{c}}, \mathrm{LI}^{\mathrm{a}, \mathrm{m}}, \mathrm{PE}^{1, \mathrm{n}}, \mathrm{WD}^{2}$,

$\mathrm{WN}^{1,8, \mathrm{a}}, \mathrm{YO}^{2}$

$\mathrm{HA}^{8,25}, \mathrm{WN}^{1}$

$\mathrm{HA}^{1,25, \mathrm{a}, \mathrm{n}}, \mathrm{PE}^{1, \mathrm{n}}, \mathrm{WN}^{1,9}$

$\mathrm{FR}^{\mathrm{a}}, \mathrm{HA}^{\mathrm{a}, 1}, \mathrm{PE}^{1}, \mathrm{WD}^{8}, \mathrm{WN}^{1,9}$

$\mathrm{HA}^{8,25}, \mathrm{PE}^{14}, \mathrm{WN}^{9}$

$\mathrm{HA}^{8}$

$\mathrm{HA}^{1,8}, \mathrm{PE}^{1,14}, \mathrm{WN}^{1}, \mathrm{YO}$

$\mathrm{HA}^{1,8}, \mathrm{LI}^{\mathrm{a}, \mathrm{m}}, \mathrm{PE}^{1}, \mathrm{WN}^{1,8,61}, \mathrm{YO}^{10}$

$\mathrm{HA}^{8,25}, \mathrm{PE}^{1}, \mathrm{WN}^{8,9}$

$\mathrm{FR}^{\mathrm{o}}, \mathrm{HA}^{1,2,8,25}, \mathrm{LI}^{\mathrm{m}}, \mathrm{PE}^{1, \mathrm{n}}, \mathrm{WD}^{2,8}$, $\mathrm{WN}^{1,8,61}$

$\mathrm{FR}^{\mathrm{a}}, \mathrm{HA}^{\mathrm{a}}, \mathrm{KN}^{\mathrm{a}}, \mathrm{LI}^{\mathrm{a}, \mathrm{c}}, \mathrm{PE}^{15, \mathrm{n}}, \mathrm{WN}^{8}$

$\mathrm{FR}^{\mathrm{s}}, \mathrm{HA}^{1,2,8,25, \mathrm{a}, \mathrm{s}}, \mathrm{SO}^{\mathrm{a}}, \mathrm{WD}^{8}, \mathrm{WN}^{8}$

$\mathrm{SO}^{2}, \mathrm{WN}^{1,2(\text { (Veit det, with "?") }}$

$\mathrm{HA}^{1,8}, \mathrm{KE}^{\mathrm{c}}, \mathrm{PE}^{14}, \mathrm{PI}^{\mathrm{c}}, \mathrm{WD}^{8}$,

$\mathrm{WN}^{26,45, \mathrm{c}}, \mathrm{YO}^{\mathrm{a}, \mathrm{o}}$

$\mathrm{HA}^{1,2,25}, \mathrm{WD}^{8, \mathrm{a}}$

$\mathrm{FR}^{\mathrm{a}}$

$\mathrm{CU}^{\mathrm{a}}, \mathrm{HA}^{9,25, \mathrm{a}}, \mathrm{PE}^{1}$

$\mathrm{PI}^{\mathrm{c}}, \mathrm{WN}^{61}$

$\mathrm{HA}^{2}, \mathrm{PI}^{\mathrm{c}}, \mathrm{YO}^{61}$ 
A.C. Dibble, F.A. Drummond, C. Stubbs, M. Veit, and J.S. Ascher

Scientific name

Sphecodes cressonii (Robertson, 1903)

Sphecodes davisii Robertson, 1897

Sphecodes dichrous Smith, 1853

Sphecodes johnsonii Lovell, 1909

Sphecodes levis Lovell and Cockerell, 1907

Sphecodes mandibularis Cresson, 1872

Sphecodes minor Robertson, 1898

Sphecodes prosphorus Lovell and Cockerell, 1907

Sphecodes ranunculi Robertson, 1897

Sphecodes solonis Graenicher, 1911

*Anthidium (Anthidium) m. manicatum (L., 1758)

Dianthidium (Dianthidium) simile (Cresson, 1864)

Stelis (Dolichostelis) louisae Cockerell, 1911

Stelis (Stelis) foederalis Smith, 1854

Stelis (Stelis) lateralis Cresson, 1864

Stelis (Stelis) nitida Cresson, 1878

Coelioxys (Boreocoelioxys) funeraria Smith, 1854

Coelioxys (Boreocoelioxys) moesta Cresson, 1864

Coelioxys (Boreocoelioxys) octodentata Say, 1824

Coelioxys (Boreocoelioxys) porterae Cockerell, 1900

Coelioxys (Boreocoelioxys) rufitarsis Smith, 1854

Coelioxys (Coelioxys) sodalis Cresson, 1878

* Megachile (Callomegachile) sculpturalis Smith, 1853

* Megachile (Eutricharaea) rotundata (Fabricius, 1793)

Megachile (Litomegachile) brevis Say, 1837

Megachile (Litomegachile) mendica Cresson, 1878

(*?)Megachile (Megachile) centuncularis (L., 1758)

Megachile (Megachile) inermis Provancher, 1888

Megachile (Megachile) montivaga Cresson, 1878

Megachile (Megachile) lapponica Thomson, 1872

[= nivalis Friese, 1903]

Megachile (Megachile) relativa Cresson, 1878

Megachile (Xanthosarus) f. frigida Smith, 1853

Megachile (Xanthosarus) gemula Cresson, 1878

Megachile (Xanthosarus) latimanus Say, 1823

Megachile (Xanthosarus) m. melanophaea Smith, 1853

Megachile (Xanthosarus) mucida Cresson, 1878

Heriades (Neotrypetes) carinata Cresson, 1864

Heriades (Neotrypetes) leavitti Crawford, 1913

Heriades (Neotrypetes) v. variolosa (Cresson, 1872)

Hoplitis (Alcidamea) albifrons albifrons (Kirby, 1837)

Hoplitis (Alcidamea) pilosifrons (Cresson, 1864)

Hoplitis (Alcidamea) producta producta (Cresson, 1864)

Hoplitis (Alcidamea) spoliata (Provancher, 1888)

$[=$ H. cylindrica $]$

Hoplitis (Alcidamea) truncata truncata (Cresson, 1878)
Counties (selected sources)

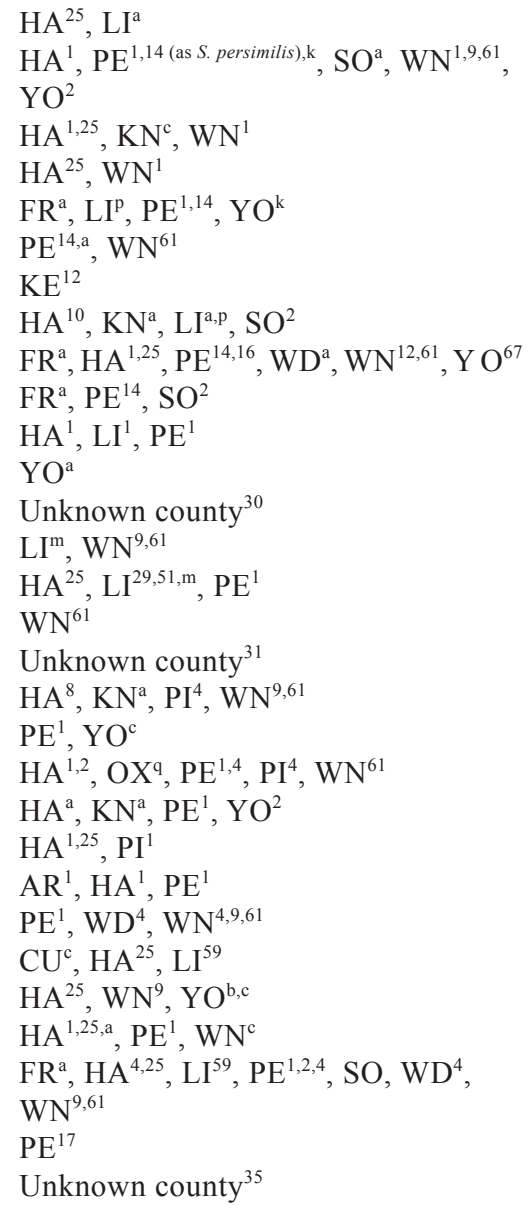

$\mathrm{AR}^{4}, \mathrm{CU}^{\mathrm{c}}, \mathrm{FR}^{\mathrm{a}}, \mathrm{HA}^{1,2,4,25, \mathrm{a}, \mathrm{o}}, \mathrm{LI}^{59}$,

$\mathrm{PE}^{1,2,4}, \mathrm{PI}^{4}, \mathrm{SO}^{2}, \mathrm{WD}^{4}, \mathrm{WN}^{1,4,9,61}, \mathrm{YO}^{\mathrm{c}}$

$\mathrm{HA}^{25, \mathrm{a}}, \mathrm{LI}^{\mathrm{a}}, \mathrm{OX}^{\mathrm{f}}, \mathrm{PE}^{1}, \mathrm{PI}^{\mathrm{a}}, \mathrm{WN}^{9}$

$\mathrm{CU}^{\mathrm{c}}, \mathrm{FR}^{\mathrm{a}}, \mathrm{HA}^{1,2,8, \mathrm{a}, \mathrm{o}}, \mathrm{LI}^{59}, \mathrm{PE}^{1,14, \mathrm{n}}$,

$\mathrm{WN}^{1,8}, \mathrm{YO}^{\mathrm{a}, \mathrm{c}}$

$\mathrm{HA}^{1,25}, \mathrm{KE}^{2, \mathrm{n}}, \mathrm{LI}^{59}, \mathrm{OX}^{\mathrm{q}}, \mathrm{PE}^{1}, \mathrm{WN}^{1}$

$\mathrm{YO}^{\mathrm{c}}$

$\mathrm{FR}^{\mathrm{a}, 57}, \mathrm{HA}^{1,25}, \mathrm{LI}^{\mathrm{a}}, \mathrm{PE}^{1,2,12}, \mathrm{SO}, \mathrm{WN}^{1,9}$,

$\mathrm{YO}^{\mathrm{c}}$

$\mathrm{WA}^{61, \text { det. Terry Griswold }}$

$\mathrm{HA}^{1,25}, \mathrm{PE}^{1,2}$

$\mathrm{SO}^{2},{ }^{29}$

$\mathrm{HA}^{1}$, Unknown county ${ }^{29}$

$\mathrm{HA}^{9}, \mathrm{WN}^{9}$

$\mathrm{HA}^{8}, \mathrm{WN}^{8}$

$\mathrm{HA}^{1,8,25}, \mathrm{PE}^{1,12,14}, \mathrm{WN}^{1,8,9,10}, \mathrm{YO}^{\mathrm{c}, \mathrm{n}}$

$\mathrm{CU}^{\mathrm{o}}, \mathrm{HA}^{25}, \mathrm{LI}^{\mathrm{m}}, \mathrm{PE}^{1,12}, \mathrm{PI}, \mathrm{WN}^{9,10,12,61}$, $\mathrm{YO}^{\mathrm{a}, \mathrm{c}}$

$\mathrm{HA}^{25}, \mathrm{PE}^{1}, \mathrm{YO}^{2}$ 
A.C. Dibble, F.A. Drummond, C. Stubbs, M. Veit, and J.S. Ascher

Scientific name

Counties (selected sources)

*Osmia (Helicosmia) caerulescens (L., 1758)

Osmia (Melanosmia) albiventris Cresson, 1864

Osmia (Melanosmia) atriventris Cresson, 1864

Osmia (Melanosmia) bucephala Cresson, 1864

Osmia (Melanosmia) collinsiae Robertson, 1905

Osmia (Melanosmia) distincta Cresson, 1864

Osmia (Melanosmia) inspergens Lovell \& Cockerell, 1907

Osmia (Melanosmia) laticeps Thomson, 1872

Osmia (Melanosmia) nigriventris (Zetterstedt, 1838)

Osmia (Melanosmia) proxima Cresson, 1864

Osmia (Melanosmia) pumila Cresson, 1864

Osmia (Melanosmia) simillima Smith, 1853

Osmia (Melanosmia) tarsata Provancher, 1888

$[=O$. kenoyeri $]$

Osmia (Melanosmia) tersula Cockerell, 1912

Osmia (Melanosmia) virga Sandhouse, 1939

Osmia (Osmia) lignaria lignaria Say, 1837

Macropis (Macropis) ciliata Patton, 1880

Macropis (Macropis) nuda (Provancher, 1882)

Melitta (Cilissa) americana (Smith, 1853)

Melitta (Cilissa) melittoides (Viereck, 1909)

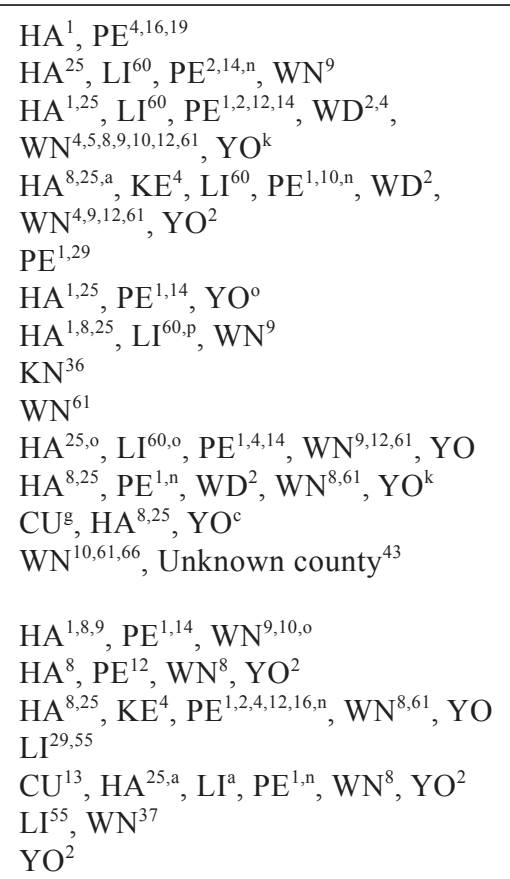

Source Legend

Code Item

1 Dibble specimens, or for Colletes compactus, photo determined by J.S. Ascher. See Dibble et al. 1997, Dibble and Drummond 1997; Roque Island 2014; Dibble and Drummond field course at Eagle Hill (2012-2016); Bee Module experiment 2012-2015, unpubl. data.

2 Veit specimens.

3 Stubbs specimens, or see Stubbs et al., 1992; Stubbs et al. 2007.

4 Drummond specimens.

5 Droege bioblitz at Acadia National Park, see Droege 2010.

6 AMNH database, includes J.S. Ascher specimens.

7 Ascher specimens and Maine Bee Type Compilation.

8 Bushmann specimens, 2010-2012 data from her Ph.D. Dissertation, and collections into 2016.

9 R. Hansen 1981, T30 MD, 1982, T4 ND, both in Washington County, and from Hancock County, see Hansen and Osgood 1983.

10 L. Guimond 1989, T32 MD, Hancock County; T31, Washington County; Deblois, Washington County; and Orono, Penobscot County.

11 Droege determinations, Nomada.

12 E.A. Osgood, including Orono, Penobscot County; Deblois, Washington County, 1961-1984; Vienna, Kennebec County, 1961-1990; Kennebunk, York County, 1961-66 (Boulanger et al. 1967), Katahdin, Piscatquis County.

13 "Me. Agr. Exp. Sta” N. Gray, Cumberland County. 1929 and 1930 (possibly C.R. Phipps).

14 Miliczky and Osgood 1979a, 1979b, Passadumkeag, Penobscot County.

15 Frederick Allen Eddy, ca. 1882 (red ink).

16 s.n., Orono, Penobscot Co., 1929 (C.R. Phipps?), 1941, 1961-1963.

17 Specimens housed in MCZ Harvard University collections . 
A.C. Dibble, F.A. Drummond, C. Stubbs, M. Veit, and J.S. Ascher

Includes specimens collected by A.E. Brower, some mention "Me. Agr. Exp. Sta.", various sites, including Orono (1930, 1936, 1938); Bar Harbor, Hancock County (1937); T19, Washington County (1962); Patten, Penobscot County (1974).

s.n. Orono 1936-1938 (C.O. Dirk?).

s.n. 17 May 1929, S. Sedgwick, Hancock County.

s.n. 1930, Orono, Penobscot County, Maine Agricultural Experiment Station.

s.n. 7 June 1961, Twp 19, Washington Co., determined as Andrena durangoensis, J.S. Ascher annotated as A. carolina.

s.n. Machias, Washington County 1929.

s.n. Maine Agricultural Experiment Station, 1961.

Procter 1938 (amplified and revised in 1946).

s.n. Maine Agricultural Experiment Station, 1930.

Bernd Heinrich, Wilton, Franklin County, n.d., assumed by Leif Richardson from Bumblebee Economics.

"Coll. E. Norton", presumably as "Maine" and no date, locale or county given.

Mitchell 1960, 1962, no county given; a few further details by taxon where available:

For Agapostemon sericeus, mapped as occurring in Maine as A. radiatus by Roberts (1972) [revision]

For Anthophora bomboides, cited by Mitchell 1960, 1962 but not by Brooks 1983.

For Osmia collinsiae, in Mitchell, but no record information indicated.

For Stelis lousiae, Parker and Bohart; recorded as extending ... north to Maine on p. 147 of Parker and Bohart, JKES 52(1) 1979, but not shown on their map.

For Coelioxys funeraria, Baker, mapped in Figure 20 of Baker 1975 with no details but shown about half way up Maine coast.

For Bombus pensylvanicus, cited in Milliron 1973 Vol. II of Monograph. Worker from Saco, York Coounty, 13 Jun 1951, collected by T.B. Mitchell.

For Hylaeus floridanus, cited by Mitchell but as H. packardi. One of the 2 specimens in type series was from Maine (described by Mitchell, 1951). Snelling (1970) proposed the synonymy and cited the Maine occurrence.

Gibbs 2011, and Jason Gibbs' determinations of Maine specimens.

Gibbs 2010, and Jason Gibbs' determinations of Maine specimens.

Gibbs et al. 2013 Evylaeus revision.

Sheffield et al. 2011, Megachile nivalis, now known as Megachile (Megachile) lapponica Thomson, 1872. Synonymy of N. American M. nivalis with European M. lapponica at http:// cjai.biologicalsurvey.ca/srpg_18/srpg_18.pdf.

For Osmia laticeps, cited in Rightmyer et al. 2010, i.e., the ZooKeys paper on non-metallic Osmia. Record is from "USA: MAINE, 15 June 1982 (1ㅇ, St. Charles)" (http://www.ncbi. nlm.nih.gov/pmc/articles/PMC3088345/).

For Melitta americana, collected by Brianne DuClos, 9 July 2013, powerline corridor, Deblois, Washington County, ME, determined by Sam Droege, GPS 44.702139, -67.989309.

Lovell, 1910.

Stephen (1954), revision of Colletes.

For Epeolus americanus, cited by Brumley 1965 [as E. americanus]; Mitchell, 1962:450; paratype of E. lanhami from "Oreno, Me."[sic]. Table 2 refers to a specimen collected by R.A. Morse and C. Zmarlicki, July 19, 1961, "Washington County", no town given.

For Holcopasites illiniosensis, cited in Hurd and Linsley 1972.

For Dufourea novaeangliae, collected in Machias (Washington County) by Samantha Gallagher July 2015.

For Pseudopanurgus aestivalis, listed by Mitchell (1960) as P. nebrascensis, see Sheffield and Perron 2014.

For Osmia tarsata, see discussion on synonymy with O. kenoyeri Cockerell, 1915, see Sheffield and Perron 2014.

Roque Island 2014, Dibble and Drummond Native Bees field course at Eagle Hill, Steuben, ME. 
A.C. Dibble, F.A. Drummond, C. Stubbs, M. Veit, and J.S. Ascher

Ribble, D.W. 1967. Revisions of 2 subgenera of Andrena: Micrandrena Ashmead and Derandrena, new subgenus (Hymenoptera: Apoidea). Ph.D. Thesis, published in 1968 as Bulletin of the University of Nebraska State Museum 8(5):237-394 .

For Andrena spiraeana, recorded from Orono (Penobscot County) by LaBerge 1973:342

LaBerge 1961 (Melissodes druriellus is former M. rustica)

LaBerge 1956

For Nomada armatella, see Mitchell $(1960,1962)$; J.S. Ascher notes the only record with details at http://www.discoverlife.org/mp/201?id=BBSL521175; specimen collected 20 May 1967, Deblois (Washington County), sin nom.

Mitchell, 1956, descriptions of Sphecodes, as "carolinus"; collected on Katahdin as "carolinus".

For Stelis lateralis, collected by D.J. Borror on 6 July 1939, no town given, Lincoln County, Ohio State U. record at http://www.discoverlife.org/mp/201?id=GBIF311451792.

Bug Guide photo.

Lovell 1922b (Bees of Maine, Part II).

Lovell 1925a (Bees of Maine, Part III).

Schwarz 1926.

Stubbs et al. 1992.

Lovell 1925b (Bees of Maine, Part IV).

Lovell 1925c (Bees of Maine, PartV).

Jennifer (Loose) Ryan, blueberry barrens collections, 1998.

Dearborn et al. 1983.

Kalyn Bickerman-Martens, Maine collections 2015.

Megan Leach, Maine collections 2014.

University of Maine Entomological Museum collection, housed in Augusta, ME.

Discover Life: within a species, click on global map to see details of voucher specimens.

s.n. Kennebunk, York County, 1960s (Boulanger et al. 1967?).

For Andrena cornelli, cited by LaBerge (1980) as A. longifacies LaBerge.

University of Maine, Orono collections 1905, 1915, collectors unknown.

Brianne DuClos, Maine collections 2014 -2015.

Discover Life, http://www.discoverlife.org/mp/201?id=AMNH_BEES24420

Discover Life, http://www.discoverlife.org $/ \mathrm{mp} / 201$ ? id=AMNH_BEES668

Symbol Abbreviation Institution or Collector

\begin{tabular}{lll}
\hline a & AMNH & American Museum of Natural History, New York, NY \\
$\mathrm{b}$ & CAES & Connecticut Agriculture Experiment Station, Storrs, CT \\
$\mathrm{c}$ & CUIC & Cornell University, Ithaca, NY \\
$\mathrm{d}$ & JML & Unknown (cited for Andrena wheeleri from Waldo County) \\
$\mathrm{e}$ & JSA & John Ascher \\
$\mathrm{f}$ & NYSM & New York State Museum, Albany, NY \\
$\mathrm{g}$ & RUAC & Rutgers University, Rutgers, NJ \\
$\mathrm{h}$ & UCD & University of California, R.M. Bohart Museum of Entomology, Davis, CA \\
$\mathrm{i}$ & UCMS & University of Connecticut, Storrs, CT \\
$\mathrm{j}$ & UMA & University of Massachusetts, Amherst, MA \\
$\mathrm{k}$ & UNHP & University of New Hampshire, Durham, NH \\
$\mathrm{l}$ & - & (left blank, could be confused with "l" [one]) \\
$\mathrm{m}$ & OSUC & Ohio State University Insect Collection, columbus, OH \\
$\mathrm{n}$ & INHS & Illinois Natural History Survey Insect Collection, Champaign, IL \\
$\mathrm{o}$ & KU & University of Kansas, Lawrence, KS \\
$\mathrm{p}$ & USNM & Smithsonian Institution, Washington, DC \\
$\mathrm{q}$ & YPM & Yale Peabody Museum, New Haven, CT \\
$\mathrm{r}$ & FMNH & Field Museum of Natural History, Chicago, IL \\
$\mathrm{s}$ & BISON & USGS Biodiversity Information Serving Our Nation (https://bison.usgs.gov) \\
\hline
\end{tabular}




\section{Results}

Sampling effort was highly uneven, and of Maine's 16 counties, only 8 have more than 50 available county records (see Fig. 1). Of these, only 5 have more than 100 bee species: Hancock County (197 confirmed species), Penobscot County (181), Washington County (162), York County (104), and Lincoln County (102). Androscoggin and Sagadahoc counties have only 11 and 8 species documented, respectively.

Despite these gaps, we list 278 described species of bees in 37 genera and 6 families for Maine (Table 2). For most of these, at least 1 voucher specimen was found or records in the literature are considered reliable. For some species (Table 2), information derived from Mitchell (1960, 1962; for 7 species) or other literature did not specify any county. A few species in Table 2 (i.e., Colletes latitarsis, Epeolus pusillus, Melissodes bimaculatus, Nomada louisianae) are mapped for Maine in Discover Life, but details about the record, including county, are pending (J.S. Ascher, unpubl. data).

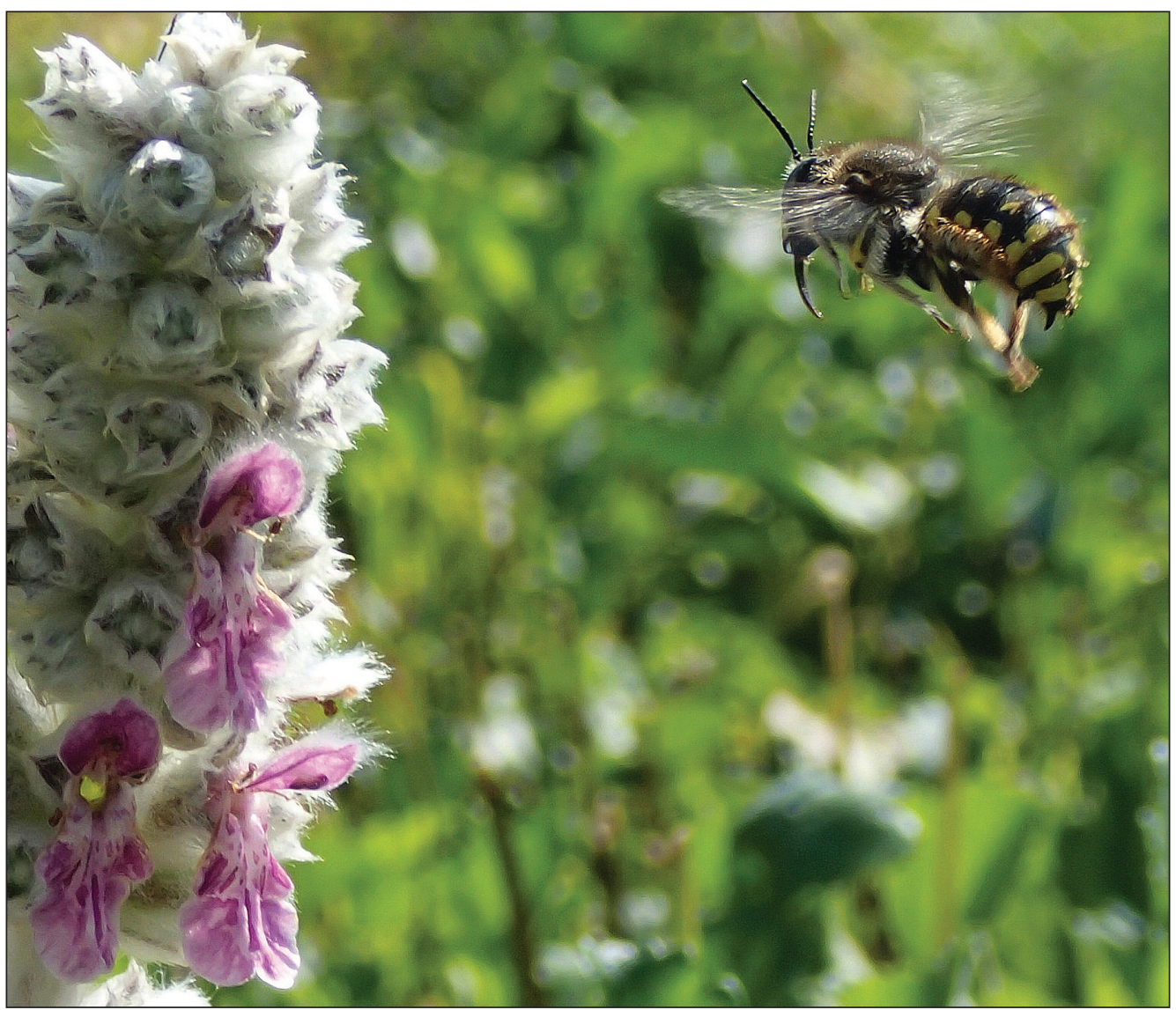

Figure 2. Non-native bee, Anthidium manicatum (European Wool-carder Bee); the males are territorial at flowers of Merostachys lanata Send (Lamb's Ears), shown here, and other plants in Old Town, Penobscot County. The male chases other bees away while he awaits a visit from a conspecific female. Photograph (C) A.C. Dibble. 
The largest genus is Andrena (53 spp.), followed by Lasioglossum (50), Nomada (28), Bombus (includes Psythirus) (17), and Osmia (16). The total count includes at least 8 non-native species of Old World origin: Apis mellifera, Lasioglossum leucozonium, L. zonulum, Andrena wilkella, Anthidium manicatum (Fig. 2), Osmia caerulescens, Megachile rotundata, and M. sculpturalis. Megachile centuncularis could be non-native (questionable status due in part to lack of confirmed records for Alaska; see Giles and Ascher 2006).

\section{Discussion}

We consider the Maine bee checklist (Table 2) to be preliminary because none of the state's 16 counties have been exhaustively sampled, despite much effort in recent studies or by some collectors. Counties with few available records may be considered to have less relative documentation, in terms of numbers of bee species (indicated by shading of counties in Fig. 1), so it is not yet possible to say with any confidence that bee diversity in one county is higher than in another. Counties in southern Maine with the warmest climate and expanses of sandy habitat are expected to have the most species, but this is not yet borne out. Penobscot County is particularly well represented because the University of Maine in Orono has been an agricultural research center since the 1860s, with entomologists active on or near campus. Early and continuing research in major Lowbush Blueberry crop areas such as Hancock and Washington counties (Yarborough 2009) have led to better exploration of the bee fauna in those regions (Bushmann and Drummond 2015).

\section{Species richness}

Bee species richness in Maine is relatively low ( $<300$ species), but not unusually so given its latitude and climate (Sheffield et al. 2003, Stubbs et al. 1996). Greater species richness was reported for bees of Wisconsin (Wolf and Ascher 2009), perhaps reflecting in part higher summer temperatures across most of that state, and much greater richness was recorded for western states such as Colorado (Scott et al. 2011). Gibbs et al. (in press) developed a checklist of 465 species in Michigan, with 38 new records. Low species richness in Maine could be due in part to the extent of forest. Maine is the most forested state in the continental US when measured as the proportion of total landscape comprised of forest vegetation landscapes (93\% of land area; Wilson and Sader 2002). Much of the Maine landscape is categorized as mixed northern hardwood, coniferous forests, and boreal spruce-fir forests (Davis 1993). Dense, shady forests are not optimal habitats for generalist bees in the region (Dibble et al., in press; Romey et al. 2007) because of insufficient floral resources and lack of open sky for insolation and navigation. Powerline rights-of-way may provide suitable open habitat for many species including regional rarities (Wagner et al. 2014). In Maine, the extent of coniferous forest with a permanently shaded understory may limit the spatial distribution and abundances of native bee communities (Groff et al. 2016), whereas in hardwood forests of southern Maine many specialist bees visit spring ephemerals in the forest understory prior to leafout. 
A.C. Dibble, F.A. Drummond, C. Stubbs, M. Veit, and J.S. Ascher

Timber harvest throughout much of Maine creates a changing light environment to which bees are likely to respond.

Native bee species richness in Maine is greatest for genera that are common across the Holarctic region and well adapted to the boreal forest and other habitats characteristic of northern latitudes (Michener 2007). Genera richest in species (Andrena, Lasioglossum, Nomada, Bombus, and Osmia) all include early emerging species that overwinter as adults. Their ecology has been a subject of the past 45 years in Maine bee research (Bushmann and Drummond 2015; Bushmann et al. 2012; Dibble and Drummond 1997; Dibble et al. 1997; Drummond et al. 2017b; Jones et al. 2014; Osgood 1972, 1989; Stubbs et al. 1992). Of the 30-35 species of Andrena that occur regularly in Maine blueberry fields (Bushmann 2013, Bushmann and Drummond 2015), the 5 most common are Andrena (Andrena) carolina and Andrena (A.) rufosignata, both of which have long malar spaces that facilitate their collection of nectar from blueberry flowers, and the generalists $A$. (Melandrena) regularis, $A$. (M.) carlini, and $A$. (M.) nivalis. These Andrena tend to be found in sandy loam soils (Osgood 1972), typified by the vast glacial plains of Washington County (Davis 1993).

Some species in the list (Table 2) are of particular interest because they are seldom collected, have a narrow distribution, were not expected to occur in the area, have been in decline, or have specific habitat requirements. There is a possibility that a taxon is under-recorded due to identification difficulties, so scarcity of records might not reflect rarity in nature. Nonetheless, one might assume that a species represented by a single recent record and 1 historic citation (Lovell 1922b) such as Melitta (Cilissa) americana, found in 2013 by Brianne DuClos, is genuinely less abundant than the many Andrena and Osmia species each represented by multiple specimens recorded from several counties.

We identified 21 species that could be considered unusual. Among recent state records, Epeoloides pilosulus (Macropis Cuckoo Bee) was found in the Kennebunk Plains in York County, June 2016 on Apocynum sp., by M. Veit along with its host Macropis nuda (known from 6 counties). Epeoloides pilosulus is the only member of its tribe in America North of Mexico, was recently rediscovered in New England (Wagner and Ascher 2008), and is now classified as endangered in Canada (COSEWIC 2011). M. Veit also found at this same site a new state record for Melitta melittoides, a rarely collected species associated with Lyonia ligustrina (L.) DC. (Maleberry; Wagner et al. 2014). In another recent example, Fenja Brodo, entomologist from Ottawa, ON, Canada, collected Holcopasites calliopsidis on Rhus typhina L. (Staghorn Sumac) on 14 July 2016 along a roadside in Steuben (Washington County).

In addition to the 4 species mentioned above, we consider others notable due to their limited representation in regional bee collections. These include Colletes consors (subspecies mesocopus), Colletes hyalinus, Colletes impunctatus, Macropis (Macropis) ciliata, Hylaeus (Hylaeus) saniculae, Hylaeus (Paraprosopis) floridanus, Hylaeus (Metziella) sparsus, Andrena (Scrapteropsis) kalmiae (newly documented for York County by M.Veit in June 2016), Melitta (Cilissa) 
americana, Heriades (Neotrypetes) leavitti, Hoplitis (Alcidamea) albifrons, Osmia (Melanosmia) inermis, Osmia (Melanosmia) laticeps, Osmia (Melanosmia) tarsata, Coelioxys (Boreocoelioxys) funeraria, Megachile (Megachile) lapponica, Bombus (Psithyrus) ashtoni (collections since 2000 are few and noteworthy), and Bombus (Bombus) affinis, which was listed as Federally Endangered in 2017 and requires careful identification.

No well-known bee species is unique to Maine, but 2 species of Nomada of uncertain status are known reliably only from holotypes from the state. Nomada proxima has uncertain taxonomic placement and status (Mitchell 1962). Nomada subrutila, described from Waldoboro in Lincoln County, has been recorded from 3 other states (Mitchell 1962), but some or all of these records may result from confusion with the widely distributed species Nomada imbricata and/or N. luteoloides. The taxonomic status of these species was only recently clarified by Schwarz and Gusenleitner (2004). Maine shares most $(95 \%+)$ of its known bee fauna with New York, Michigan, Massachusetts, and Connecticut. New Brunswick, Canada, has similar climate, soils, and vegetational communities to Maine (Griffin et al. 2009, Smit et al. 2007), and studies of Lowbush Blueberry there (Javorek et al. 2002) record a bee fauna similar to that of comparable sites in Maine (Boulanger et al. 1967, Javorek et al. 2002, Stubbs et al. 1992). Certain species differ, notably Triepeolus brittaini Cockerell, which is now well known from all 3 Maritime Provinces of Canada, with numerous recent records (J.S. Ascher, S.K. Javorek [Agriculture and Agri-Food Canada, Kentville, NS, Canada], and J. Klymko, [Nature Serve, Atlantic Canada Conservation Data Centre, Sackville, NB, Canada], unpubl. data), but has not yet been recorded elsewhere. The bee fauna of Nova Scotia is also similar to that of Maine (Sheffield et al. 2003), but includes remarkable disjunct occurrences of "southern" species such as Colletes willistoni Robertson, that have not been recorded from Maine (though M. Veit has collected C. willistoni in Massachusetts and New Hampshire, and it could be in Maine). The influence of warming by the Gulf Stream and other ocean currents may be more extensive on Nova Scotia (Boughner 1937) than on Maine, and may explain these remarkable distributions.

Bee species other than those included in our checklist have been proposed by various sources to occur in Maine. We excluded the following because of insufficient documentation or problematic identification: Andrena (Andrena) cornelli Viereck, A. (Gonandrena) fragilis Smith, A. (Trachandrena) heraclei Robertson, A. (Tylandrena) perplexa Smith, A. (Gonandrena) platyparia Robertson, A. (Micandrena) ziziae Robertson, Lasioglossum (Dialictus) tegulare Robertson (most if not all records pertain to L. ellisiae), Nomada lehighensis Cockerell (see Droege 2010), and N. subnigrocincta Swenk. All of these species could plausibly occur in the state, but we have not been able to definitively confirm their presence. The taxonomic status of several additional cleptoparasitic Nomada "cuckoo bee" species and morphospecies remains uncertain pending completion of ongoing taxonomic revision of the genus (Droege et al. 2010). We have excluded a number of other records of species that cannot plausibly occur as far northeast as Maine, such as $\mathrm{Au}$ gochloropsis sumptuosa (Smith), a species reported for Maine by Mitchell (1960) 
but recorded reliably no nearer than New Jersey, and Ceratina metallica H.S. Smith (syn. C. strenua), reported by Procter (1946) but likely to have been misidentified.

\section{Life history and ecology}

Life histories for most of the bees found in Maine are derived from studies conducted elsewhere and assumed to apply in Maine, but some bee species have been studied using Maine populations: Osmia atriventris (Drummond and Stubbs 1997a), Andrena crataegi (Osgood 1989), and Bombus impatiens (Drummond 2012a, Stubbs and Drummond 2001).

Regarding sociality and cleptoparasitism (one bee species lays its eggs in the nest of another bee species), 135 species in the Maine checklist (Table 2) are solitary bees, 39 are eusocial, 55 species are cleptoparasitic (e.g., Nomada and other Nomadinae, and Coelioxys) or socially parasitic in the case of some halictines (e.g., Sphecodes). Four species of Bombus (Psithyrus) are social parasites of other bumble bees.

Our checklist suggests that $22.7 \%$ of the total known bee species in Maine are cleptoparasites or social parasites of pollen-collecting bees. Bushmann and Drummond (2015) reported a similar percentage in a more recent survey of the bee community associated with Lowbush Blueberry in Maine. Their 4-year study involved 44 sites in Hancock, Waldo, and Washington counties. They found that $17.7 \%$ of bee species richness and $4.8 \%$ of the total bee abundance associated with Lowbush Blueberry in Maine are cleptoparasites. The number of bee cleptoparasites found in Maine is similar in species richness but is about one-fourth the relative abundance compared to that found by Sheffield et al. (2013) in Canada (varied from 1 to $10 \%$ cleptoparasite species relative to bee species richness and up to $22 \%$ in terms of individual cleptoparasite bee numbers relative to total bee abundance). Actual parasitism rates are not known for the Maine bee fauna. Over North American bee fauna, with respect to species richness, rate of cleptoparasitism might vary between 0 and $91 \%$ in individual bee species, and could be as high as $29 \%$ at the community level, based on a review of the literature (Wcislo 1996).

About half of the 278 bee species (Table 2) are known or suspected to be soil-nesting bees, including all species of Andrena and Colletes and most Lasioglossum. One Andrena species found in Maine, A. crataegi, is known to interconnect tunnels between individuals and may form a large communal ground nest in which each solitary sister bee is a queen (Osgood 1989). Of bees listed in Table 2, forty-three species nest in cavities and or stems, including several species of Lasioglossum in subgenus Dialictus that excavate nests in soft wood (Michener 2007), as do Auglochlora pura and Anthophora terminalis. Tiny bees in the genus Ceratina (small carpenter bee) may exploit an existing hole in a stem to access the hollow or pithy interior in which they lay their eggs. Examples of Maine plant species associated with stem-nesting bees are Rubus spp. (blackberry and raspberry), Sambucus spp. (elderberry), and Rhus typhina L. (Staghorn Sumac). Bees that nest in wood include Xylocopa virginiana (Eastern Carpenter Bee), which can excavate galleries in wooden structures and may cause some damage. Megachile 
A.C. Dibble, F.A. Drummond, C. Stubbs, M. Veit, and J.S. Ascher

and Osmia can nest in holes in stumps, logs, or standing dead trees, and might use holes made by boring beetles.

Bee species differ in their overwintering condition and time of emergence, with important consequences for pollination of Maine crops (Bushmann 2013). Queen bumble bees overwinter as adults that emerged and mated during the previous autumn, while most other native bees emerge as fully developed adults for their maiden flight in spring or summer, from nests established and provisioned by their mothers during the previous season (or earlier that same season). In most native Maine bee species other than Bombus, males typically emerge first, a condition called protandry, and they are ready to mate when the females appear. Emergence is staggered depending on the bee species, with early bees appearing with first flowers of Salix spp. (willow) and Acer rubrum L. (Red Maple) in spring (Bushmann 2013). The importance of willow to bees active in early spring was studied by Ostaff et al. (2015) and was documented in a common garden study in Maine by A.C. Dibble, F.A. Drummond, and L. Berg Stack (unpubl. data).

Some Bombus, especially the currently common species B. ternarius (Tricolored Bumble Bee; Fig. 3) and B. vagans (Half-black Bumble Bee), are in evidence from early spring (the beginning of April or, recently, in warm years, as early as late March) until late October. The large queens can be seen foraging on flowers

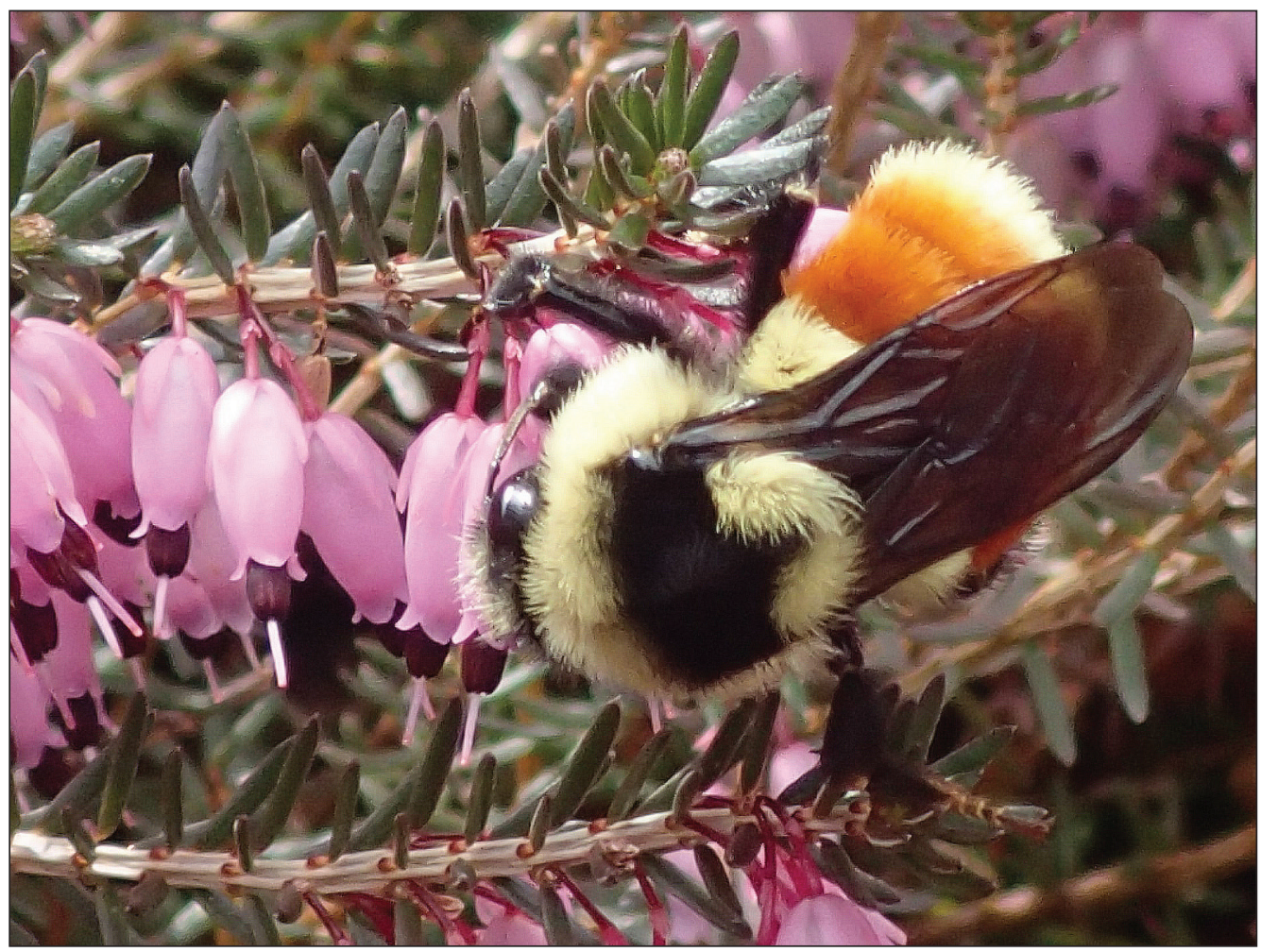

Figure 3. Queen Bombus ternarius (Tricolored Bumble Bee) on flowers of Erica tetralix L. (Crossleaf Heath) in a garden, on 24 April 2014, Brooklin, Hancock County. Photograph (C) A.C. Dibble. 
A.C. Dibble, F.A. Drummond, C. Stubbs, M. Veit, and J.S. Ascher

into June; new queens are observed as early as late July (Bushmann 2013). Bumble bee workers of various sizes can be found starting in June and then throughout the growing season, with average size of individuals increasing gradually over the summer and fall (Bushmann et al. 2012).

An especially early bee is Colletes inaequalis, which often emerges before the snow has fully melted from its nest aggregations. Other bees active early in spring include many species in the genera Andrena, Lasioglossum, Nomada, and Osmia (Fig. 4). Adult emergence can begin as early as late March, i.e., in southern Maine in a particularly early spring, but more typically in April (A.C. Dibble, pers. observ.; Bushmann and Drummond 2015; Stubbs et al. 1992).

Bees with long flight seasons (both univoltine and multivoltine) extending from spring until fall include primitively eusocial halictine species, e.g., in the genera Halictus, Lasioglossum (Fig. 5), and Augochlorella, and the subsocial carpenter bees, e.g., genera Ceratina and Xylocopa. Most Colletes (Fig. 6) and their Epeolus cleptoparasites, and Melissodes and their Triepeolus cleptoparasites, fly from summer to fall in association with peak bloom of plants in the family Asteraceae such as asters and goldenrods. Early emerging and late-flying species and most specialists have restricted flight seasons. Data for Maine on flight activity of most species is in the process of being summarized (E. Venturini and F.A.

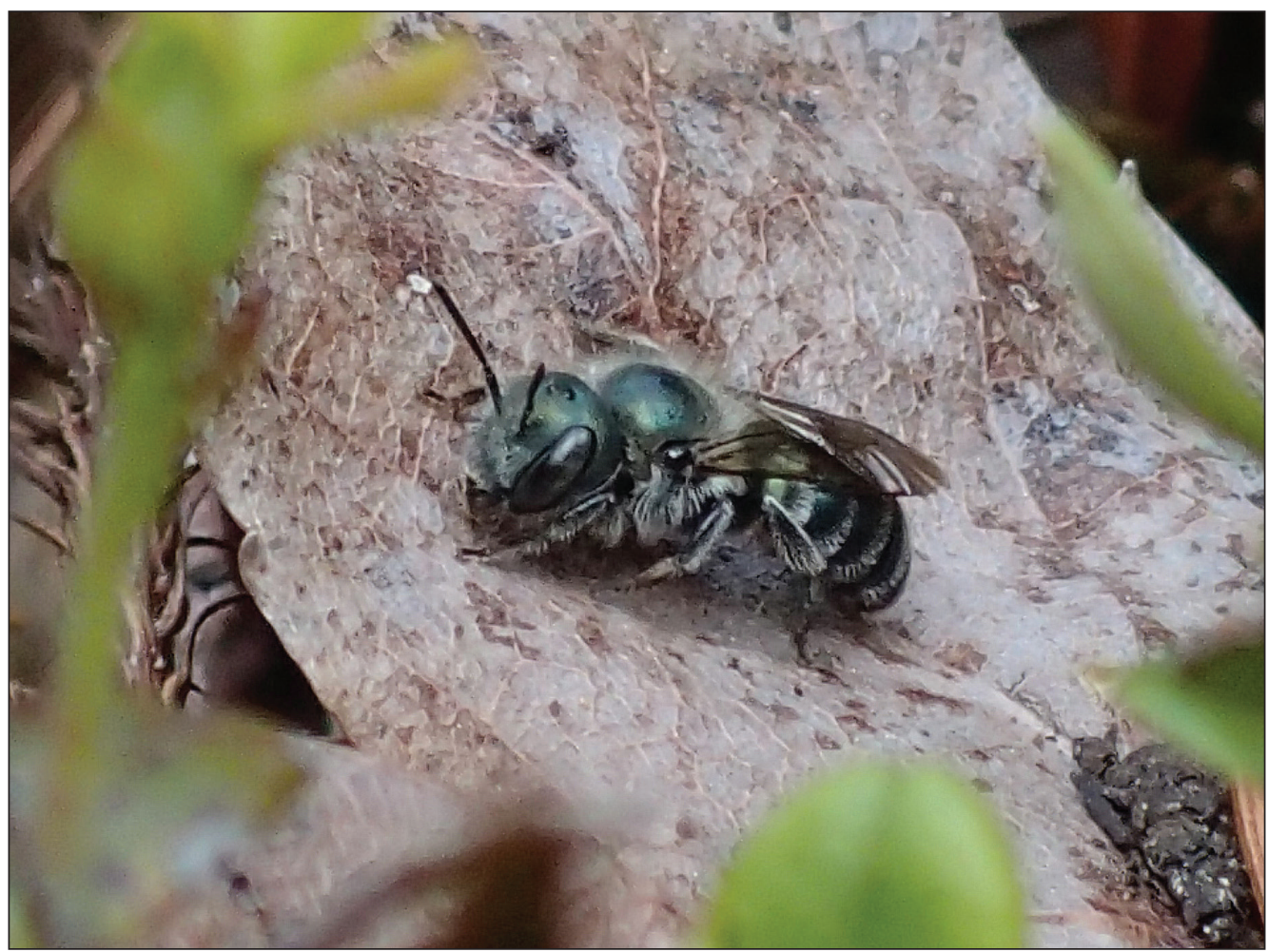

Figure 4. Female Osmia (mason bee) rests briefly on a leaf while foraging on Vaccinium vitis-idaea L. (Northern Mountain Cranberry), in Brooklin, Hancock County, 13 June 2015. Photograph (C) A.C. Dibble. 
A.C. Dibble, F.A. Drummond, C. Stubbs, M. Veit, and J.S. Ascher

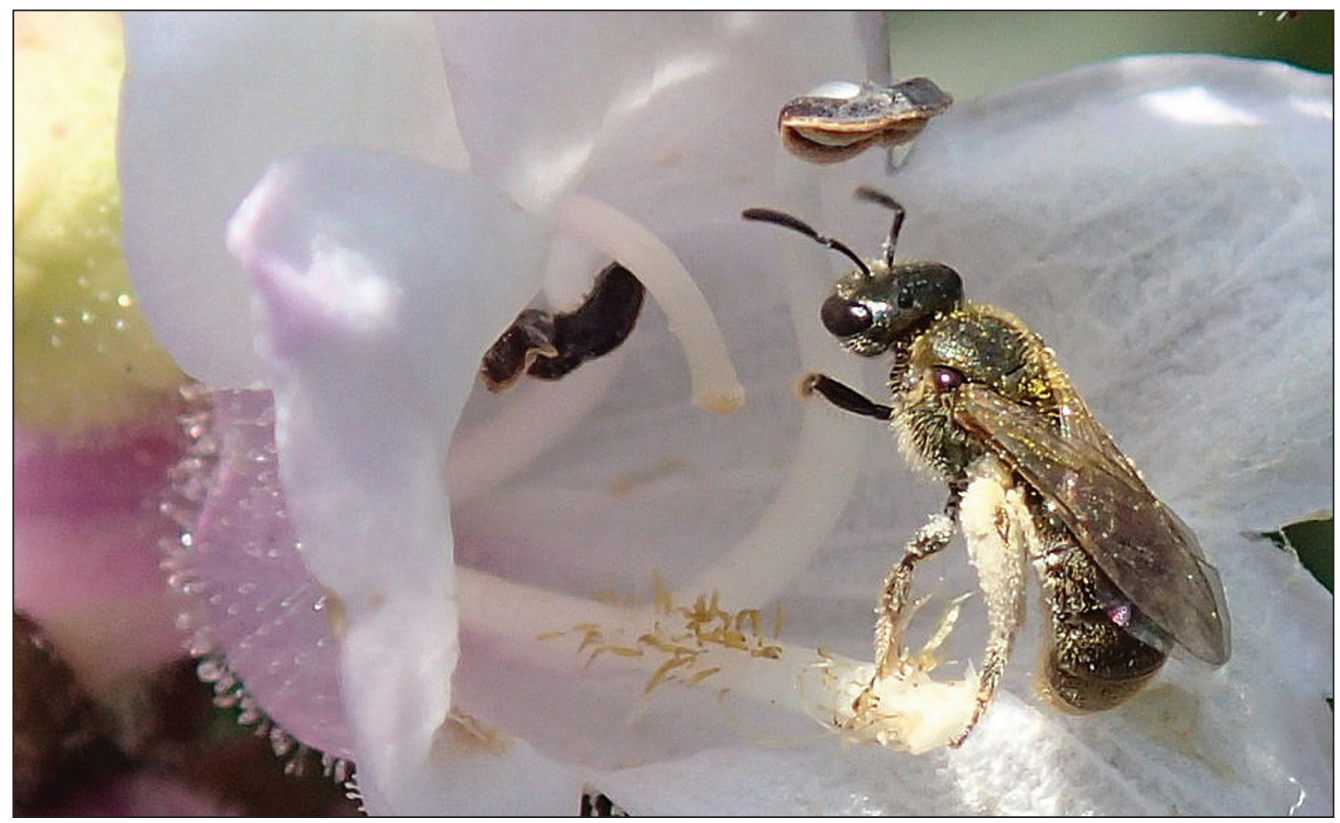

Figure 5. Lasioglossum (Dialictus) sp. (a metallic sweat bee) female on flowers of Penstemon digitalis "Mystica" (Foxglove Beardtongue), 8 July 2014, Blue Hill, Hancock County. Photograph (C) A.C. Dibble.

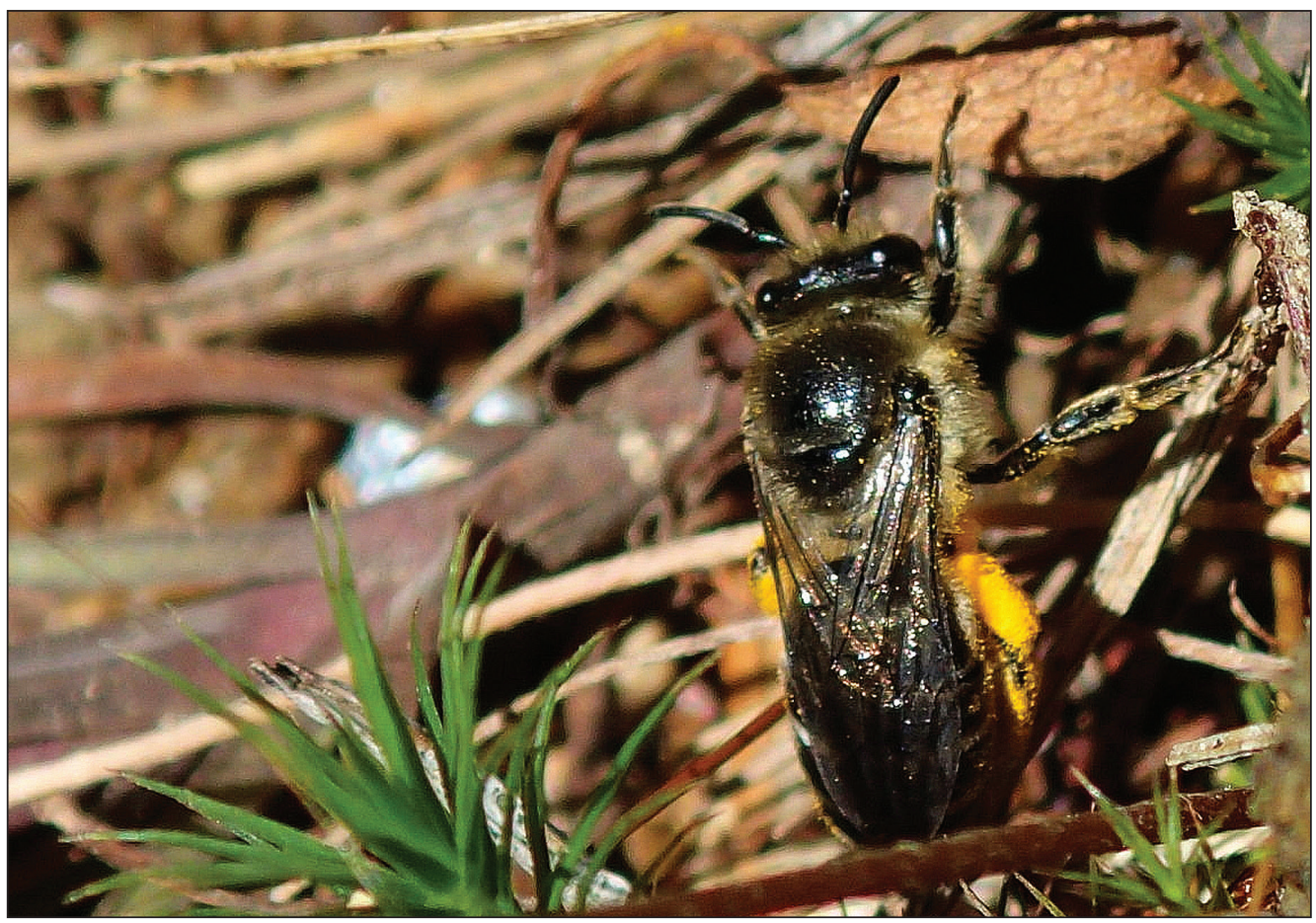

Figure 6. Colletes compactus compactus (Cellophane Bee) female, late-flying solitary bee species seldom collected in Maine, at her nest entrance, 16 September 2011, Brooklin, Hancock County. Photograph (C) A.C. Dibble. 
A.C. Dibble, F.A. Drummond, C. Stubbs, M. Veit, and J.S. Ascher

Drummond, University of Maine, Orono, ME, unpubl. data). Many species were included in phenological diagrams for offshore islands of New York State (Ascher et al. 2014) and Massachusetts (Goldstein and Ascher 2016). Flight activity varies across sites and years. Likewise, flight activity of most Osmia species peaks in May-June, but collections of $O$. atriventris and $O$. simillima have been made in Maine as late as August in Hancock and Washington counties (Droege 2012). Some bee species, including several Megachile and their Coelioxys cleptoparasites, are aestival, i.e., most active in summer (A.C. Dibble and F.A. Drummond, pers. observ.; Droege 2012). Colletes and its Epeolus cleptoparasites can be active in mid-September in Hancock and Washington counties (A.C. Dibble, unpubl. data).

Information on host-plant usage includes Stubbs et al.'s (1992) reports of pollen and nectar records for native bee species associated with Lowbush Blueberry. Osmia atriventris is considered polylectic, but in a study in Winterport, ME, where 54 species of flowering plants came into bloom during its nesting period, individuals collected pollen from only a few species-90\% was ericaceous pollen, most likely of Lowbush Blueberry (Drummond and Stubbs 1997b). Subsequently Bushmann and Drummond (2015) studied flower use and pollen collection by native bees during Lowbush Blueberry bloom, and found that in addition to ericaceous plants, the 3 most common native forage plant species or genera were Cornus canadensis L. (Bunchberry), Rubus spp. (raspberry and blackberry), and Houstonia caerulea L. (Azure Bluet). Fowler (2016) reviewed regional patterns of bee specialization on plants of the northeastern US.

Lowbush Blueberry is one of the native plants on which floral visitors have been studied intensively (Bushmann and Drummond 2015, Drummond et al. 2017b, Stubbs et al. 1992, and numerous other papers). Others include Amelanchier (shadbush; Dibble and Drummond 1997, Dibble et al. 1997), Viburnum nudum spp. cassinoides (L.) Torr. \& A. Gray (Withe-rod; Miliczky and Osgood 1979b, Stubbs et al. 2007), and Spiraea alba var. latifolia (Aiton) H.E. Ahles (White Meadowsweet; Stubbs et al. 2007).

Other than in such pollinator studies, forage-plant information from museum collections of bees is often lacking or may be unreliable. Emphasis on data regarding flowering-plant associations will enhance our ability to protect or manipulate habitat for native bees and can lead to Maine-specific recommendations with potential usefulness in other parts of northeastern North America. The most up-todate information on Maine forage plants and larger-scale vegetational landscapes can be found in Bushmann and Drummond (2015); Dibble and Drummond (1997); Dibble et al., in press; Droege (2012); Drummond et al. (2017a); Groff et al. (2016); Stubbs et al. (1992); and Venturini et al. (2015). More Maine studies are in the pipeline (A.C. Dibble, F.A. Drummond, and L. Berg Stack, unpubl.data). These kinds of data, when considered with reference to regional reviews of specialist bees (Fowler 2016), will increase the effectiveness of pollinator plant lists (e.g., A.C. Dibble, unpubl. data; Ley et al. 2011; Venturini et al. 2015) so that bee gardens and pollinator strips are more likely to accomplish their intended goals in Maine. 


\section{Changes in abundance}

Long-term population dynamics of bee communities have not been well studied in Maine, other than climate-change data reported by Drummond et al. (2017b). In that study, continuous sampling over a 29-year period in a Lowbush Blueberry field in Winterport, Waldo County, resulted in total bee abundance varying 2-3 fold from one year to the next. Bee categories (Bombus, Andrena, Osmia, and "Halictids and Other") were not highly correlated with one another and showed independence. Osmia declined since 2007, but the total bee community abundance was stable during the 29-year time period. Predictability from models developed by Drummond et al. (2017b) was low to moderate and suggested that abundance fluctuations depend on both density-dependent factors and stochastic density-independent factors such as weather.

The relative abundance of wild native Bombus species in Maine has changed dramatically since the early 1960s (Bushmann et al. 2012). Whereas the most common native bees in Maine continue to include bumble bee species such as Bombus ternarius (Fig. 3) and B. vagans, both in the subgenus Pyrobombus, another once-common species, B. terricola (Yellow-banded Bumblebee) in subgenus Bombus, and its social parasite $B$. ashtoni (treated by Williams et al. 2014, as conspecific with the Old World Bombus bohemicus) have declined in Maine (Bushmann et al. 2012; Heinrich and Heinrich 1983a, b) and across the region (Bartomeus et al. 2013, Cameron et al. 2011, Kerr et al. 2015). Bombus affinis (Rusty-patched Bumble Bee), also parasitized by $B$. ashtoni, appears to have been relatively common at one time in Maine and is recorded for 10 counties, but today it is seldom found. It was noted by Procter (1946) as scarce for Mount Desert Island. Boulanger et al. (1967) listed B. affinis from New Brunswick but did not specify any Maine counties. On the other hand, several species of subgenus Pyrobombus such as B. ternarius and especially B. impatiens (Eastern Bumble Bee) have increased (Bushmann and Drummond 2015). Surveys of B. terricola distribution in Maine in 2014-2015 indicate a resurgence of that species at many sites (Drummond 2015), but not of its social parasite B. ashtoni.

\section{Habitat and landscapes}

Using literature and specimen-label data to assess relative sampling effort by habitat, we found studies in blueberry fields to be well represented, and edge habitats associated with mixed conifers were also relatively well studied. Many habitats in Maine require more attention, including coastal islands, well-drained sandy soils on islands and elsewhere, hardwood forests, swamps, bogs, open mountain summits, roadsides, and urban and suburban plantings. Bees on coastal islands in New England are of particular interest because they potentially include relictual or disjunct species (see Goldstein and Ascher 2016) or those that have potentially declined on the mainland due to infection by a microsporidian pathogen, Nosema bombii Fantham and Porter (Bushmann et al. 2012). On a 1-day visit in late July 2014 to Roque Island, ME, 6 Bombus species were documented including B. fervidus (Yellow Bubmblee), which is relatively scarce in Washington County, but not B. impatiens (A.C. Dibble, unpubl. data). In visits to Monhegan Island, ME, and when studying samples in the Cornell University Insect Collections from Appledore 
A.C. Dibble, F.A. Drummond, C. Stubbs, M. Veit, and J.S. Ascher

Island, ME, we found no strikingly unusual species. Counties in southern Maine with sandy soils, such as York and Cumberland, could be under-represented in the checklist, and harbor bees such as Nomada tiftonensis that have a restricted distribution in the state. Additional bee species characteristic of northern climates have been found on Mount Washington in New Hampshire and may also extend their ranges southward into mountainous habitats in Maine, but surveys of the most promising high-elevation sites in Maine, such as Katahdin, are lacking. Bees may be genuinely scarce there. No bees other than a single Bombus terricola were seen on a 4-day hiking trip to a lowland forest area near Katahdin in August 2014 (A.C. Dibble, pers. observ.). In a scenario of upward-shifting treeline with climate change, open habitats that support unusual montane bee faunas in the state could change greatly in coming decades due to upslope advance of coniferous trees (Dibble et al. 2009), putting some as yet unstudied cold-adapted bee species at risk (Kerr et al. 2015) before they have been inventoried.

\section{Conservation concerns}

Native bees of Maine could encounter competition with non-native bees for floral resources or nest sites. They could be impacted by pesticides, natural enemies, diseases, habitat degradation (through invasive plant encroachment), habitat fragmentation, and climate change (Brown and Paxton 2009, Goulson et al. 2015, Kerr et al. 2015).

Non-native bee species can be adventive, or they could arrive in Maine as purposeful introductions. Non-native bees can alter ecosystems in subtle but significant ways (Goulson 2003, Goulson et al. 2015). They may compete for floral resources and nest sites, spread pests and pathogens to native bee populations, and effect diminishing plant repoduction in native plant species that depend on native bee pollinators but are not visited much by introduced bees.

The best-known and most economically important managed non-native bee in Maine is Apis mellifera (European Honey Bee), which has been in North America since 1622 (Kingsbury 1906). Since 1630, when Maine was a part of Massachusetts, Apis mellifera has been established in the state (Martin et al. 1980). It has been used in Lowbush Blueberry pollination since the 1950s (Lee 1958), with managed colonies supplemented by feral honey bees, but since the 1990s persistent overwintering feral hives are seldom found in Maine (F.A. Drummond, unpubl. data). The disappearance of feral colonies is a phenomenon that has been observed across the entire US and is attributed to the accidental introduction of the parasitic mite Varroa destructor (Delaplane 2001). Colony Collapse Disorder in managed Apis mellifera demonstrates the collective impact of multiple simultaneous threats (Drummond 2012c, Ellis et al. 2010, Neumann and Carreck 2010, Ratnieks and Carreck 2010). This syndrome, which became evident in 2006, prompted new research on pollinator habitat quality as a means of enhancing existing native bee populations in Maine, with the idea that native bees might be required to play an increasing role as pollinators of Lowbush Blueberry and other Maine crops (Asare 2013, Venturini et al. 2015). High rates of honey bee colony losses (averaging greater than $30 \%$; Lee et al. 2015, vanEngelsdorp and Meixner 2010) across the US since 2006 have 
A.C. Dibble, F.A. Drummond, C. Stubbs, M. Veit, and J.S. Ascher

resulted in severe economic hardship for commercial beekeepers. Honey bee colonies continue to be reared in Maine and brought from out of state (more than 75,000 commercial colonies per year; Drummond 2012c). Many of these colonies swarm and produce initial feral colonies that last only 2-3 years in the absence of management (Drummond et al. 2012). Apis mellifera is present in all Maine counties, though voucher specimens could not be found for some counties. A recent survey among Maine beekeepers (most are assumed to be hobbyists) to obtain pollen for analysis of pesticide residue from honey bee colonies will be reported separately. Any counties not vouchered were filled into Table 2 based on beekeeper responses to that survey (E. Ballmann, University of Maine, Orono, ME, unpubl.data).

Bombus impatiens is both a native bumble bee (categorized as such in Table 2) and the species that is purchased from commercial suppliers and brought into Maine in colonies (quads) annually for blueberry, Malus pumila Miller (Apple), Solanum lycopersicum L. (Tomato), and Curcurbitaceae (curcurbits) pollination in both the greenhouse and in the field (Drummond 2012a). Several thousand colonies are set out each year in Maine Lowbush Blueberry fields (Drummond 2012a, Stubbs et al. 2001). Bombus impatiens abundance and collecting frequency was lower in Maine prior to the 1990s compared to more recent times (Bushmann et al. 2012). Current populations of $B$. impatiens found in Maine could have multiple sources including both managed commercial colonies brought from Michigan (Drummond 2012a, Stubbs and Drummond 2001, Stubbs et al. 2001), and wild native genotypes with populations that appear to be expanding their range northward in possible association with climate change (Bushmann et al. 2012). Recent collections in more than 200 locations from 2011-2015 have shown that B. impatiens individuals make up $\sim 10-20 \%$ of bumble bee individuals in Maine (F.A. Drummond, unpubl. data). Bushmann et al. (2012) showed that relative abundance of B. impatiens in the 1960s was much lower than current estimates in Maine (1961-1963: not detected, 19971998: 1-4\%, 2010-2015: 10-20\%). Procter (1946) did not record B. impatiens in coastal Hancock County.

Megachile rotundata (Alfalfa Leaf-cutter Bee) was used commercially as a managed pollinator for Lowbush Blueberry in Maine for more than a decade in the 1990s through to the early 2000s (Stubbs et al. 1997b) but not subsequently (F.A. Drummond, pers. observ.). Many hundreds of thousands of prepupae in individual leaf cells were imported from the western US and Canada and released by Maine growers for pollination of the Lowbush Blueberry crop (Stubbs and Drummond 1997a, b, c; Stubbs et al. 1997a, b). The bee was commonly trap nested and observed in blueberry fields in the 1990s when releases were made in Maine. Intensive bee surveys conducted from 2010 to 2012 using diverse sampling methods (Bushmann and Drummond 2015) in the same regions as these earlier releases have not recorded a single individual of $M$. rotundata, suggesting that it may be poorly adapted to longterm establishment at these sites (Stubbs and Drummond 1997a, b, c).

Several other non-native megachilids are adventive in the New World and may be a detriment to native species (Roulston and Malfi 2012, Strange et al. 2011). Megachile sculpturalis (Giant Asian Resin Bee) was detected in the southeastern 
US in 1994 and has been dispersing rapidly across the US (Hinojosa-Díaz 2008, Mazurkiewicz 2010). It was collected in Portland, ME, by M. Mazurkiewicz in 2008, and by A.C. Dibble and S. Bushmann in Aroostook County in 2013, with additional documentation since. Anthidium manicatum (European Wool-carder Bee) males defend flowers of Merostachys lanata Send (Lamb's Ears; Fig. 2), Agastache foeniculum (Anise Hyssop), and horticultural bedding plants in the genus Salvia (Mealy Sage) against other floral visitors in Maine, and they attack bumble bees that attempt to visit such flowers (Gibbs and Sheffield 2009). Anthidium manicatum appears to be increasing in numbers and locales in Maine (A.C. Dibble, pers. observ.). Osmia caerulescens (Blue Mason Bee) may be associated with urban habitats, and has been found in Maine as early as the 1930s (see sources cited in Table 2). Osmia lignaria (Blue Orchard Bee), indicated in Table 2 as non-native, has been recommended as a managed bee for orchards (Torchio 1976); in Maine it is relatively scarce. Osmia (Osmia) cornifrons (Radoszkowski) (The Horned faced Bee) was deliberately introduced from East Asia by USDA scientists (Yamada et al. 1971) and is abundant farther south in eastern North America, but has not yet been documented in Maine. Changes in abundance over time for some species of Megachilids were studied by Drummond et al. (2017b).

Additional small-bodied non-native bees could have negligible influence on native bees, but this relationship has not been measured. A mining bee, Andrena wilkella, and 2 sweat bees, Lasioglossum leucozonium and L. zonulum, are examples of ground-nesting bees native to Europe that are found in the Northeast and are documented for Maine. In general, with the exception of Apis mellifera, non-native bees appear to be increasing in richness and abundance in Maine, but they still comprise a far lower proportion than in states to the south, such as New York (see Matteson et al. 2008). Some possible explanations for this difference include Maine's colder climate, more limited international trade, and limited extent of cities and suburbs (Simberloff 2013).

Pesticide exposure may not be as important a threat to native bee health in Maine as in other states where crops are routinely treated, e.g., with imidacloprid, a widely used, systemic neonicotinoid (one class of persistent insecticides thought to harm beneficial insects even at sublethal doses; Blacquiere et al. 2012). Coating of crop seed with neonicotinoids is not much in use in the state compared to levels applied to Brassica napus L. (Oilseed Rape) crops in the United Kingdom, Hungary, and Germany (Woodcock et al. 2017) and Zea mays subsp. mays L. (Corn) in Ontario and Quebec, Canada (Tsvetkov et al. 2017). Even so, exposure in Maine crops can be at levels that impact local bee populations as demonstrated in a study on honey bees by Drummond (2012c). Exposure of native bees to pesticides and the resulting effects are complicated and not well studied. As an example, a recent study by Ciarlo et al. (2012) showed that the "inert ingredients" in pesticide formulations have negative effects on honey bee learning. Studies of managed pollinators such as Apis mellifera, Bombus, Osmia, and Megachile suggest either significant negative effects (Drummond 2012b, Ladurner et al. 2008, Laycock et al. 2012) or no measurable impact (Drummond 
A.C. Dibble, F.A. Drummond, C. Stubbs, M. Veit, and J.S. Ascher

$2012 b, c)$. No true consensus has emerged with all of the conflicting data that currently exist, especially for the neonicotinoids, though recent studies suggest that site differences affect exposure rates to bees and subsequent impacts (Tsvetkov et al. 2017, Woodcock et al. 2017). In Maine, the effects of pesticides on native bees have not been extensively researched. Drummond and Stubbs (1997a) showed a negative relationship between the amount of insecticide active ingredient applied during the Lowbush Blueberry growing season and the relative abundance of Osmia spp. in blueberry fields. Ten percent of the variation in Osmia density was attributed to pesticide exposure. Bushmann (2013) in a 3-year study of native bee communities in more than 40 blueberry fields did not find evidence to suggest increased pesticide use affected native bee abundance or richness. Natural population fluctuations of bee communities between years and locations make it difficult to assess the impacts of pesticide exposure, and pesticides continue to be a serious potential concern.

Natural enemies of native bee pollinators in Maine include cleptoparasitic bees such as Nomada cuckoos of Andrena species (Bushmann and Drummond 2015), parasitoid wasps in the genus Monodontomerus (Torymidae), and the bee flies (Bombyliidae). Myopa spp. (white-faced flies) (Conopidae) attack Bombus in flight and lay their eggs in the abdomen of the living host. Velvet ants (Hymenoptera: Mutillidae) are found in Maine blueberry fields (Jones et al. 2014) where they parasitize soil-nesting bees and wasps. Crab spiders in the genus Thomisus (Thomisidae) camouflage on flowers and attack bees when they arrive to forage. Although such pests can thwart efforts to enhance populatons of native species as pollinators (Cane et al. 1996), these species and their interactions might also be considered indicators of a healthy or naturally functioning ecosystem and bee community (Sheffield et al. 2013). Perspective might shift according to management goals. Drummond and Stubbs (1997a) found that Osmia spp. (mostly O. atriventris) populations nesting in artificial nest blocks had $\sim 20 \%$ parasitization by aculeate wasps after 4 years of nest block utilization in a blueberry field in Winterport. Presence of these pest wasps might not be considered an index of a commercially healthy bee community, at least from a blueberry farmer's perspective. On the other hand, lack of Stelis bee cleptoparasites may indicate a disturbed system or unhealthy host-bee populations (see Sheffield et al. 2013).

A question remains regarding whether some exotic or cosmopolitan pathogens might be contracted by native bees from managed bees brought in to pollinate Lowbush Blueberry or other crops. Bushmann et al. (2012) found that Nosema bombi, a microsporidian pathogen of Bombus associated with blueberry fields, varied in its infection rate according to the bumble bee species, with a much higher rate in B. terricola - a species that had been in documented decline throughout its range - than in other common bumble bees. There was no correlation between farms employing the use of commercial bumble bees (B. impatiens) for pollination of Lowbush Blueberry and localized infection rates. Nonetheless, this is an example in which a threat can be more lethal to some species of native bees than to their congeners. 
Another potential threat to native bees is encroachment into native habitats by non-native invasive plants (Pimentel et al. 2005, Stubbs et al. 2007, Weber 2017). Although many invasive plants in Maine do attract native bees (A.C. Dibble, unpubl. data; Stubbs et al. 2007), their presence can be considered a detriment because they displace native plants with which native bees evolved. We think it is possible that habitats dominated by invasive plants have lower diversity of pollen and nectar resources and subsequently a narrower range of overlapping flowering phenologies that support native bees. Stubbs et al. (2007) tested the supposition that invasive plants can become sinks for pollinators that would otherwise pollinate flowers of native plant species, leading to lower fruit set in the native flora. They found that pollination and bee abundance were not much affected by presence of 2 invasive plants, Lythrum salicaria L. (Purple Loosestrife) and Rhamnus frangula L. (Glossy Buckthorn), in Acadia National Park, but flowering period for Berberis thunbergii DC. (Japanese Barberry) overlapped that of Lowbush Blueberry and attracted floral visitors, mostly native bees, to such an extent that significantly lower floral visitation rates were observed on Lowbush Blueberry in the vicinity of barberry patches. In another example, Purple Loosestrife, a perennial of wet soils, attracts generalist bees (in Eurasia, where native, it also attracts specialists) but displaces native vegetation (Stubbs et al. 2007). Among the numerous bee-visited native plants that could be affected by Purple Loosestrife is Lysimachia, the oil and pollen source for Macropis (in turn, the only host of the associated cleptoparasitic genus Epeoloides). When meadows and roadsides become dominated by invasive Lupinus polyphyllus Lindl. (Bigleaf Lupine), then Asclepias syriaca L. (Common Milkweed) has less habitat. The latter is much more attractive to diverse native bees, including numerous Bombus species, and other insects such as Danaus plexippus (L.) (Monarch Butterfly), for which milkweed is a host for the larval stage. Bigleaf Lupine emerges earlier in spring than does Common Milkweed, and quickly shades and outcompetes any Common Milkweed that is already present.

Other examples of invasive plants that could impact Maine bees by reducing floral resources are: (1) herbs such as Impatiens glandulifera Royle (Ornamental Jewelweed or Himalayan Balsam), Hypericum perforatum L. (St. John's-wort), and Fallopia japonica (Houtt.) Ronse Decr. (Japanese Knotweed); (2) grasses such as Phragmites australis (Cav.) Trin. ex Steud. (Common Reed) and Phalaris arundinacea L. (Reed Canary Grass) (but pollen of nonnative Phleum pratense L. [Common Timothy] is gathered by bees; Rivernider et al., in press); (3) tender and woody vines such as Solanum dulcamara L. (Bittersweet Nightshade) and Celastrus orbiculatus Thunb. (Oriental Bittersweet); (4) shrubs including Japanese Barberry, Elaeagnus umbellata Thunb. (Autumn Olive), and Lonicera morrowii A. Gray (Morrow's Honeysuckle); and (5) trees including Acer platanoides L. (Norway Maple) and Robinia pseudoacacia L. (Black Locust). Most of these examples, but not all, are on the Maine Department of Agriculture, Conservation, and Forestry Invasive Plant List (http://www.maine.gov/dacf/mnap/features/invasive_plants/ invsheets.htm). 
Habitat changes apart from invasive plant encroachment, that could impact bee populations, include agricultural practices, urbanization, and forest succession. Bee responses to most of these are not fully quantified in Maine. Venturini et al. (2017) found that pollinator plantings for native bees in association with Lowbush Blueberry agroecosystems increased abundance of native bees. This finding suggests that diverse gardens in cities and towns are also beneficial to bee diversity and abundance in Maine (Dibble et al., in press), but data are lacking. Regarding forest succession, Miliczky (1978) found 65 bee species at edges and small openings in a Picea (spruce)-Abies (fir) forest, suggesting that areas adjacent to or gaps within closed-canopy conifer stands are not necessarily devoid of bee communities. It appears likely that in additon to anthropogenic alterations to the landscape, fire, floods, and catastrophic wind-throw can be associated with early successional openings in which bees find suitable habitats. Native Americans in Maine burned small openings for centuries (Cronon 1983); presumably they altered habitats near waterways and maintained blueberry-dominated openings in conifer and mixed hardwood stands. Beginning ca. 1605, European colonists conducted what we might consider a vast deforestation (Cronon 1983), and brought in Apis mellifera and nonnative plants, some of which became important bee forage (e.g., Taraxacum officinale F.H. Wigg. [Common Dandelion]). Populations of many native bee species had potential to expand in response to increased open sky and additional forage and ground-nesting opportunities. Beginning in the the mid-1800s, large areas of agricultural fields were abandoned in Maine and many other parts of New England, and overall the trend in vegetation composition in uplands continues today toward forest-dominated ecosystems (Dibble et al. 2008, Wessels 1997). The Penobscot Experimental Forest of the University of Maine in Bradley, Penobscot County, is an example of such a forested landscape. It is dominated by Picea rubens Sarg. (Red Spruce) and other conifers, and some stands within this forest are periodically harvested in patches under various cutting regimes. The landscape features 343 plant species (Dibble 2013) of which about $59 \%$ offer pollen and nectar resources that might be taken by bees. Timber harvest in Maine continues, leading to large openings that favor bees in the short term; this is a landscape shift that, along with habitat fragmentation and urbanization, alters bee habitat resources, and not necessarily for the worse (Romey et al. 2007). In patches, ongoing transition through succession of edge habitats to closedcanopy forest may reduce bee diversity and abundance temporarily. Would bee communities then resemble those that were present prior to intense anthropogenic habitat alteration? This cannot be measured, and we do not know precisely what bee diversity may have been lost, but we can compare to historic collections and reasonably interpret Maine's current bee fauna as an artifact of human disturbance and its current diversity as indicative of the capacity of these animals to adapt and exploit opportunity, demonstrating resilience in a changing landscape.

Climate change and in particular hotter summer temperatures may already be resulting in changes to Bombus ranges in Maine and elsewhere (Kerr et al. 2015). Campbell et al. (2009) speculated that climate change will result in wetter conditions in Northeastern North America. Rainy springs, as documented by Drummond 
et al. (2017b), and summers could detrimentally affect bees of Maine and other regions of northern New England in several ways: (1) upset a synchrony between bloom period for host plants and active period for native bees (but see Bartomeus et al. 2011), (2) limit good weather conditions for foraging with the result that insects are unable to provision sufficient brood for subsequent generations, and (3) lead to prolonged conditions that enhance fungal infections in soil-nesting bees (Batra et al. 1973).

Data from Drummond et al. (2017b) indicate that climate change that might affect bee activity and foraging during the spring bloom period of Lowbush Blueberry is already in effect in Maine. Their estimate of pollination days for Blue Hill in Hancock County between 1960 and 2015 showed a significant increase in the average number of pollination days as of 1990 but then a decline through to 2015. They ascribed this trend to an increase in rainy weather during bloom since 1990. There are potential impacts on crop pollination and on long-term bee population dynamics. Lack of synchrony between bees and their host plants could lead to an inadequacy of pollen and nectar sources (Miller-Rushing and Primack 2008) that might impact bee populations. A recent study (Bartomeus et al. 2011) showed that bee responses were comparable to and less extreme than the response of relevant plant species.

\section{Conclusions}

Maine has a diversity of native bees typical for northeastern North America but with fewer species than states with warmer climates such as those to the south and west. Native bee species richness is rather high for typically Holarctic genera that overwinter as adults and are otherwise well adapted to a cold temperate climate (Michener 2007). While Apis mellifera feral colonies are in decline, other nonnative bees could be increasing in species richness and abundance.

The preliminary county checklist focuses attention on gaps in available data, with the goal of informing future inventories. Additional sampling is needed in western, northern, and southern Maine, with particular attention to Androscoggin, Somerset, and Sagadahoc counties, along with other counties from which few species have been recorded.

Many vouchers we examined are from studies of pollinators for Lowbush Blueberry. Similarly intensive surveys of other crops (Apple, Vaccinium macrocarpon Aiton [American Cranberry], Highbush Blueberry, curcurbits) are needed in Maine. Crops that are pollinator-independent might also be surveyed for associated bees, as for Solanum tuberosum L. (Potato) in Michigan (Buchanan et al. 2017). Natural habitats that could be targeted for more intensive sampling include well-drained sandy openings, coastal islands, and higher elevations with features such as tablelands and balds, among other Maine plant communities (Gawler and Cutko 2010). Our assumption that York and Cumberland County sandy areas could have high diversity compared to other counties may be confounded by the extent to which habitats are being developed in that part of the state, where changed land use, intensive lawn management, or forest succession occupy areas that were formerly openings with abundant flowers. Bee 
associations with vegetation dominated by heaths (Ericaceae, e.g., Vaccinium) such as bogs, and other types of wetlands (Anderson and Davis 1998, Davis 1993) need further survey.

New collections should be subjected to identification by expert taxonomists. We urge that if regional bee faunas are to be fully useful assessments then they must be based on careful consideration of taxonomy and behavior (e.g., host plants and habitat associations), with attention to known biogeographic patterns (Goldstein and Scott 2015). Cane (2001) cautioned that ecological studies require correct bee identifications for their validity. Bee label data should include floral records so that host-plant associations and phenology can be summarized, and specimens should ideally include preservation of associated pollen loads for morphological and metagenomic studies. We hope that this first checklist of the bees of Maine can serve as a baseline for measuring the effects of anticipated climate and habitat changes on native and exotic bee populations in coming decades.

\section{Acknowledgments}

We appreciate funding from the US Department of Agriculture National Institute of Food and Agriculture (Specialty Crops Research Initiative Contract/Grant/Agreement No. 2011-51181-30673), the University of Maine, the American Museum of Natural History, and Stewards LLC. This project was supported by USDA National Institute of Agriculture, Hatch Project number ME0-21505 through the Maine Agricultural and Forest Experiment Station. We thank people who, in addition to J.S. Ascher. and M. Veit, examined bees for this report: (1) Jason Gibbs, Cornell University and Michigan State University, identified Lasioglossum (Dialictus) specimens from the Maine Entomological Museum; (2) Terry Griswold and Wallace E. LaBerge identified Osmia captured in Lowbush Blueberry fields in 1990 and 1997-1998, and Jason Gibbs determined Osmia captured in 2010-2012; (3) Sam Droege, USGS Pawtuxent National Wildlife Refuge, identified Nomada and many other Maine bees; and (4) Leif Richardson, University of Vermont, identified hundreds of Maine Bombus specimens. Charlene Donahue of the Maine Forest Service provided records from the Maine Forest Service Insect Collection, Augusta, ME. We appreciate data, insights, and numerous county and some state records from Sara Bushmann regarding particularly Bombus and Lasioglossum in Maine. We are grateful to recent and current researchers who shared data with us, with special thanks to Jennifer (Loose) Ryan, Eric Venturini, Kalyn Bickerman-Martin, and Brianne Du Clos. Judith Collins assisted in many ways. We appreciate help from Anne Favolise, Hadel Go, and Keith Goldfarb in preparing Figure 1. Comments received from Dave Halliwell, Keith Goldfarb, and 2 anonymous reviewers on earlier drafts significantly improved this monograph. Thank you to John Pickering for maintaining the biodiversity portal www. discoverlife.org. We recognize the many contributions of bee experts from past decades who have collected, determined, or annotated Maine bee specimens; their observations, skill, and dedication made this report possible. This is Maine Agricultural and Forest Experiment Station Publication number 3566. Comments from 4 anonymous reviewers improved an earlier version of the manuscript.

\section{Literature Cited}

Anderson, D.S., and R.B. Davis. 1998. The flora and plant communities of Maine peatlands. Technical Bulletin 170. University of Maine Agriculture and Forestry Experiment Station, Orono, ME. 98 pp. 
A.C. Dibble, F.A. Drummond, C. Stubbs, M. Veit, and J.S. Ascher

Asare, E. 2013. The economic impacts of bee pollination on the profitability of the Lowbush Blueberry industry in Maine. M.Sc. Thesis, School of Economics, The University of Maine, Orono, ME. 167 pp.

Ascher, J.S., and J. Pickering. 2017. Discover Life bee species guide and world checklist (Hymenoptera: Apoidea: Anthophila). Draft-47. Available online at http://www.discoverlife.org/mp/20q?guide=Apoidea_species. Accessed 27 June 2017.

Ascher, J.S., S. Kornbluth, and R.G. Goelet. 2014. Bees (Hymenoptera: Apoidea: Anthophila) of Gardiners Island, Suffolk County, New York. Northeastern Naturalist 21:47-71.

Baker, J.R. 1975. Taxonomy of five nearctic subgenera of Coelioxys (Hymenoptera: Megachilidae). University of Kansas Science Bulletin 50:649-730.

Bartomeus, I., J.S. Ascher, D.L. Wagner, B.N. Danforth, S.R. Colla, S. Kornbluth, and R. Winfree. 2011. Climate-associated phenological advances in bee pollinators and beepollinated plants. Proceedings of the National Academy of Sciences of the United States of America 108(51):20645-20649.

Bartomeus, I., J.S. Ascher, J. Gibbs, B.N. Danforth, D.L. Wagner, S.M. Hedtke, and R. Winfree. 2013. Historical changes in northeastern United States bee pollinators related to shared ecological traits. Proceedings of the National Academy of Sciences of the United State of America 110:4656-4660.

Batra, L.R., S.W.T. Batra, and G.E. Bohart. 1973. The mycoflora of domesticated and wild bees. Mycopathologia et Mycolagia Applicata 49(1):13-44.

Bickerman-Martens, K., B. Swartz, R. Butler, and F. A. Drummond. 2017. Documenting the diversity, distribution, and status of Maine bumble bees: The Maine Bumble Bee Atlas and citizen scientists. Maine Policy Review 26(2):43-49.

Blacquiere, T., G. Smagghe, C.A. van Gestel, and V. Mommaerts. 2012. Neonicotinoids in bees: A review on concentrations, side-effects, and risk assessment. Ecotoxicology 21(4):973-92.

Boughner, C.C. 1937. The climate of Canada. Quarterly Journal of the Royal Meteorological Society 63(271):419-432.

Boulanger, I.W., G.W. Wood, E.A. Osgood, and C.O. Dirks. 1967. Native bees associated with Low-bush Blueberry in Maine and eastern Canada. Maine Agricultural Experiment Station Bulletin T 26, Technical Series. Orono, ME. 22 pp.

Bouseman, J.K., and W.E. LaBerge. 1978. A revision of the bees of the genus Andrena of the Western Hemisphere. Part IX. Subgenus Melandrena. American Entomological Society, Transactions 104(3/4):275-389.

Brooks, R.W. 1983 Systematics and bionomics of Anthophora: The bomboides group and species groups of the New World. The University of California Publications in Entomology $98: 1-84$.

Brown, M.J.F., and R.J. Paxton. 2009. The conservation of bees: A global perspective. Apidologie 40(3):410-416.

Brumley, R.L. 1965. A revision of the bee genus Epeolus Latreille of western American north of Mexico. M.Sc. Thesis. Utah State University, Logan, UT. 92 pp.

Buchanan, A. L., J. Gibbs, L. Komondy, and Z. Svendrei. 2017. Bee community of commercial Potato fields in Michigan and Bombus impatiens visitation to neonicotinoid-treated Potato plants. Insects 8(1):30. Available online at doi:10.3390/insects8010030.

Bushmann, S.L. 2013. Wild bee (Hymenoptera: Apoidea) communities associated with the Lowbush Blueberry agroecosystem of Maine. Ph.D. Dissertation. University of Maine, Orono, ME. 137 pp. 
A.C. Dibble, F.A. Drummond, C. Stubbs, M. Veit, and J.S. Ascher

Bushmann, S.L., and F.A. Drummond. 2015. Abundance and diversity of wild bees (Hymenoptera: Apoidea) found in Lowbush Blueberry growing regions of Downeast Maine. Environmental Entomology 44:1-15.

Bushmann, S.L., F.A. Drummond, L.A. Beers, and E. Groden. 2012. Wild bumblebee (Bombus) diversity and Nosema (Microsporidia: Nosematidae) infection levels associated with Lowbush Blueberry (Vaccinium angustifolium) production and commercial bumblebee pollinators. Psyche 2012:1-11.

Cameron, S.A., Hines H.M., and P.H. Williams. 2007. A comprehensive phylogeny of the bumble bees (Bombus). Biological Journal of the Linnean Society 91:161-188.

Cameron, S.A., J.D. Lozier, J.P. Strange, J.B. Koch, N. Cordes, L.F. Solter, and T.L. Griswold. 2011. Patterns of widespread decline in North American bumble bees. Proceedings of the National Academy of Sciences of the United States of America 108:662-667.

Campbell, J.L., L.E. Rustad, E.W. Boyer, S.F. Christopher, C.T. Driscoll, I. J. Fernandez, P. M. Groffman, D. Houle, J. Kiekbusch, A.H. Magill, M.J. Mitchell, and S.V. Ollinger. 2009. Consequences of climate change for biogeochemical cycling in forests of northeastern North America. Canadian Journal of Forest Research 39(2):264-284.

Cane, J.H. 2001. Habitat fragmentation and native bees: A premature verdict? Conservation Ecology 5(1):3. Available online at http://www.consecol.org/vol5/iss1/art3/. Accessed 27 June 2017.

Cane, J.H., D. Schiffhauer and L.J. Kervin. 1996. Pollination, foraging, and nesting ecology of the leaf-cutting bee, Megachile (Delomegachile) addenda (Hymenoptera: Megachilidae) on cranberry beds (Vaccinium macrocarpon). Annals of the Entomological Society of America 89:361-367.

Ciarlo T.J., C.A. Mullin, J.L. Frazier, and D.R. Schmehl. 2012. Learning impairment in honeybees caused by agricultural spray adjuvants. PLoS ONE 7(7):e40848. Available online at doi:10.1371/journal.pone.0040848.

Committee on the Status of Endangered Wildlife in Canada (COSEWIC). 2011. COSEWIC assessment and status report on the Macropis Cuckoo Bee Epeoloides pilosulus in Canada. Ottawa, ON, Canada. ix +25 pp. Available online at http://www.registrelep-sararegistry. gc.ca/document/default_e.cfm?documentID=2297. Accessed 17 November 2017.

Covell, C.V., Jr. 1972. A catalog of the J.H. Lovell types of Apoidea (Hymenoptera), with lectotype designations. Proceedings of the Entomological Society of Washington 74:10-18.

Cresson, E.T. 1863. List of the North American species of Bombus and Apathus. Proceedings of the Entomological Society of Philadelphia 2:83-116.

Cronon, W. 1983. Changes in the Land: Indians, Colonists, and the Ecology of New England. Hill and Wang, New York, NY. 241 pp.

Davis, D.R., and F.G. Hevel. 1995. Donation of the Auburn E. Brower collection to the Smithsonian Institution. Journal of the Lepidopterists' Society 49(3):253-254.

Davis, R.B. 1993. The Natural History of Maine. Jokers are Wild Publishing House, Orono, ME. 157 pp.

Dearborn, R. G., R. Bradbury, and G. Russell, 1983. The forest insect survey of Maine: Order Hymenoptera. Maine Forest Service, Augusta, ME. 101 pp.

Delaplane, K.S. 2001. Varroa destructor: Revolution in the making. Bee World 82(4):157-159.

Dibble, A.C. 2013. Vascular flora of the Penobscot Experimental Forest, with provisional lists of lichens and bryophytes. Pp 103-137 In L.S. Kenefic and J.C. Brissette (Comps.). Penobscot Experimental Forest: 60 years of research and demonstration in Maine, 1950-2010. General Technical Report NRS-P-123. US Department of Agriculture, Forest Service, Northern Research Station, Newtown Square, PA. Available online at http:// www.fs.fed.us/nrs/pubs/gtr/gtr_nrs-p-123.pdf. Accessed 27 June 2017. 
A.C. Dibble, F.A. Drummond, C. Stubbs, M. Veit, and J.S. Ascher

Dibble, A.C., and F.A. Drummond. 1997. Floral syndrome in Amelanchier nantucketensis (Rosaceae) II. Bee preference and diversity associated with andropetaly. Canadian Journal of Botany 75:1860-1867.

Dibble, A.C., F.A. Drummond, and W.E. LaBerge. 1997. Floral syndrome in Amelanchier nantucketensis (Rosaceae). I. Floral density, bee activity, and characterization of andropetaly. Canadian Journal of Botany 75:1851-1859.

Dibble, A.C., K. Zouhar, and J. Kapler-Smith. 2008. Chapter 5. Northeastern bioregion, Pp. 61-90, In K. Zouhar, J. Kapler-Smith, M.L. Brooks, and S. Sutherland (Eds.). Wildland fire in ecosystems: Fire and nonnative invasive plants. General Technical Report RMRS-GTR-42-vol. 6. US Department of Agriculture, Forest Service, Rocky Mountain Research Station, Ogden, UT. Available online at http://www.fs.fed.us/rm/pubs/ rmrs_gtr042_6.pdf. Accessed 27 June 2017.

Dibble, A.C., N.G. Miller, J.W. Hinds, and A.M. Fryday. 2009. Lichens and bryophytes of the alpine and subalpine zones on Katahdin, Maine, I: Overview, ecology, climate, and conservation aspects. The Bryologist 112(4):651-672.

Dibble, A.C., A.L. Averill, K. Bickerman-Martens, S.C. Bosworth, S. Bushmann, F.A. Drummond, A.K. Hoshide, M.E. Leach, K. Skyrm, E. Venturini, and A. White. In press. Bees and their habitats in four New England states. Maine Agricultural and Forest Experiment Station Publication, University of Maine, Orono, ME.

Droege, S. 2010. The very handy bee manual. 2015 revision. Available online at http://bio2. elmira.edu/fieldbio/beemanual.pdf. Accessed 27 June 2017.

Droege, S. 2012. Report on bees collected during the Schoodic Peninsula Hymenoptera blitz, Acadia National Park, 30 July-1 Aug 2010. Pp. 35-36, In D. Chandler, D. Manski, C. Donahue, and A. Alyokhin (Eds.). Biodiversity of the Schoodic Peninsula: Results of the insect and arachnid bioblitzes at the Schoodic District of Acadia National Park, Maine. Maine Agricultural and Forest Experiment Station, Technical Bulletin 206. Orono, ME. 210 pp.

Droege, S., M.G. Rightmyer, C.S. Sheffield, and S.G. Brady. 2010. New synonymies in the bee genus Nomada from North America (Hymenoptera: Apidae). Zootaxa 2661:1-32.

Drummond, F.A. 2012a. Commercial bumble bee pollination of Lowbush Blueberry. International Journal of Fruit Science 12(1-3):54-64.

Drummond, F.A. 2012b. Effect of Imidacloprid on bumble bees: Cage trial, 2009. Arthropod Management Tests 37(1):C8. Available online at http://dx.doi.org/10.4182/ amt.2012.C8.

Drummond, F.A. 2012c. Effect of Assail on honeybees, 2008. Arthropod Management Tests 37(1):C7. Available online at http://dx.doi.org/10.4182/amt.2012.C7.

Drummond, F.A. 2015. Rebound of a once common bumble bee. Presentation at the Northeast Natural History Conference. Springfield, MA. Available from the author.

Drummond, F.A., and C.S. Stubbs. 1997a. Potential for management of the Blueberry Bee, Osmia atriventris Cresson. Proceedings of the Sixth International Symposium on Vaccinium Culture. Acta Hort 446:77-86.

Drummond, F.A., and C.S. Stubbs. 1997b. Sampling bee populations in Lowbush Blueberry in Maine. Proceedings of the Sixth International Symposium on Vaccinium Culture. Acta Hort 446:101-108.

Drummond, F.A., and C.S. Stubbs. 2003. Wild bee conservation for wild blueberry fields. University of Maine Cooperative Extension Fact Sheet 630. Orono, ME. 12 pp. 
A.C. Dibble, F.A. Drummond, C. Stubbs, M. Veit, and J.S. Ascher

Drummond, F.A., K. Aronstein, J. Chen, J. Ellis, J. Evans, N. Ostiguy, W. Sheppard, M. Spivak, and K. Visscher. 2012. The first two years of the stationary hive project: Abiotic site effects. American Bee Journal 152:369-374. Available online at http://articles. extension.org/pages/63773/the-first-two-years-of-the-stationary-hive-project:-abioticsite-effects.

Drummond, F., E. Ballman, and J. Collins. 2017a. Are they weeds or a life force? Or sustainability on the edge. Spire, The Maine Journal of Conservation and Sustainability 2017 issue. Available online at https://umaine.edu/spire/2017/05/04/drummond-et-al/.

Drummond, F.A., A.C. Dibble, C. Stubbs, S. Bushmann, J.S. Ascher and J. Ryan. 2017b. A Natural History of Change in Native Bees Associated with Lowbush Blueberry in Maine. Northeastern Naturalist 24 (Monograph 15):49-68.

Ellis, J.D., J.D. Evans, and J. Pettis. 2010. Colony losses, managed colony population decline, and Colony Collapse Disorder in the United States. Journal of Apicultural Research 49(1):134-136.

Fowler, J. 2016. Specialist bees of the Northeast: Host plants and habitat conservation. Northeastern Naturalist 23(2):305-320.

Franklin, H.J. 1911. New North American Bombidae. Transactions of the American Entomological Society 37:157-168.

Franklin, H.J. 1912. The Bombidae of the New World. Transactions of the American Entomological Society 38:177-486.

Gawler, S., and A. Cutko. 2010. Natural Landscapes of Maine: A Guide to Natural Communities and Ecosystems. Maine Natural Areas Program, Augusta, ME. 293 pp.

Gibbs, J. 2010. Revision of the metallic species of Lasioglossum (Dialictus) in Canada (Hymenoptera, Halictidae, Halictini). Zootaxa 2591:1-382.

Gibbs, J. 2011. Revision of the metallic Lasioglossum (Dialictus) of eastern North America (Hymenoptera, Halictidae, Halictini). Zootaxa 3073:1-216.

Gibbs, J., and C.S. Sheffield. 2009. Rapid range expansion of the Wool-carder Bee, Anthidium manicatum (Linnaeus) (Hymenoptera: Megachilidae), in North America. Journal of the Kansas Entomological Society 82(1):21-29.

Gibbs, J., L. Packer, S. Dumesh, and B.N. Danforth. 2013. Revision and reclassification of Lasioglossum (Evylaeus), L. (Hemihalictus), and L. (Sphecodogastra) in eastern North America (Hymenoptera: Apoidea: Halictidae). Zootaxa 3672:1-117.

Gibbs, J., J.S. Ascher, M.G. Rightmyer, and R. Isaacs. In press. The bees of Michigan (Hymenoptera: Apoidea: Anthophila), with notes on distribution, taxonomy, pollination, and natural history. Zootaxa.

Giles, V., and J. S. Ascher. 2006. A survey of the bees of the Black Rock Forest Preserve, New York (Hymenoptera: Apoidea). Journal of Hymenoptera Research 15:208-231.

Goldstein, P.Z., and J.S. Ascher. 2016. Taxonomic and behavioural composition of an island fauna: A survey of bees (Hymenoptera: Apoidea: Anthophila) on Martha's Vineyard (Dukes County, Massachusetts, USA). Proceedings of the Entomological Society of Washington 118(1):37-92.

Goldstein, P.Z., and V.L. Scott. 2015. Taxonomic and behavioral components of faunal comparisons over time: The bees of Boulder County past and present (Colorado, USA) (Hymenoptera: Anthophila). Proceedings of the Entomological Society of Washington. 117(3):290-346.

Goulson, D. 2003. Effects of introduced bees on native ecosystems. Annual Review of Ecology, Evolution, and Systematics 34:1-26. 
A.C. Dibble, F.A. Drummond, C. Stubbs, M. Veit, and J.S. Ascher

Goulson D., E. Nicholls, C. Botias, and E. Rotheray. 2015. Bee declines driven by combined stress from parasites, pesticides, and lack of flowers. Science 347(6229):1255957. DOI 10.1126/science.1255957. Available online at http://www.moraybeedinosaurs. co.uk/neonicotinoid/Bee $\% 20$ declines $\% 20$ driven $\% 20$ by $\% 20$ combined $\% 20$ stress $\% 20$ from $\% 20$ parasites $\% 20$ pesticides $\% 20$ and $\% 201$ ack $\% 20$ of $\% 20$ flowers.pdf. Accessed 27 June 2017.

Graenicher, S. 1905. Some observations on the life history and habits of parasitic bees. Bulletin of the Wisconsin Natural History Society 3:153-167.

Graenicher, S. 1911. Bees of northwestern Wisconsin. Bulletin of the Public Museum of the City of Milwaukee 1:221-249.

Graenicher, S. 1914. Wisconsin bees of the genus Perdita. Canadian Entomologist 46:51-57.

Graenicher, S. 1927. On the biology of the parasitic bees of the genus Coelioxys. Entomological News 38:231-235, 273-276.

Graenicher, S. 1935. Bee fauna and vegetation of Wisconsin. Annals of the Entomological Society of America 28:285-310.

Griffin, T., G. Anderson, F. Drummond, E. Groden, W. Honeycutt, J. Jemison, L. Stack, and D. Yarborough. 2009. Agriculture. Pp. 39-42, In G. Jacobson, I.J. Fernandez, P.A. Mayewski, and C.V. Schmitt (Eds.). Maine's Climate Future: An Initial Assessment. University of Maine, Orono, ME. 70 pp.

Groff, S.C., C.S. Loftin, F.A. Drummond, S. Bushmann, and B. McGill. 2016. Parameterization of the InVEST crop pollination model to spatially predict abundance of wild blueberry (Vaccinium angustifolium Aiton) native bee pollinators of Maine, USA. Environmental Modelling Software 79:1-9.

Hall, I.V., L.E. Aalders, N.L. Nickerson, and S.P. Vander Kloet. 1979. The biological flora of Canada. 1. Vaccinium angustifolium Ait., Sweet Lowbush Blueberry. Canadian FieldNaturalist 93:415-430.

Hansen, R.W., and E.A. Osgood. 1983. Insects visiting flowers of wild Red Raspberry in spruce-fir forested areas of eastern Maine. Entomological News 94(4):71-76.

Heinrich, B. 1971. Temperature regulation in the sphinx moth Manduca sexta. Journal of Experimental Biology 54:141-152.

Heinrich, B. 1972a. Temperature regulation in the bumblebee Bombus vagans: A field study. Science: 175:183-187.

Heinrich, B. 1972b. Energetics of temperature regulation and foraging in a bumblebee, Bombus terricola Kirby. Journal of Comparative Physiology 77:49-64.

Heinrich, B. 1972c. Patterns of endothermy in bumblebee queens, drones, and workers. Journal Comparative Physiology 77:65-79.

Heinrich, B. 1972d. Physiology and brood incubation in the bumblebee queen, Bombus vosnesenskii. Nature 239:223-225.

Heinrich, B. 1973. The energetics of the bumblebee. Scientific American 228:96-102.

Heinrich, B. 1974a. Thermoregulation in bumblebees. I. Brood incubation by Bombus vosnesenskii queens. Journal of Comparative Physiology 88:129-140.

Heinrich, B. 1974b. Pheromone-induced brooding behavior in Bombus vosnesenskii and B. edwardsii (Hymenoptera: Bombidae). Journal of the Kansas Entomological Society 47:396-404.

Heinrich, B. 1975. Thermoregulation in bumblebees. II. Energetics of warm-up and free flight. Journal of Comparative Physiology 96:155-166. 
A.C. Dibble, F.A. Drummond, C. Stubbs, M. Veit, and J.S. Ascher

Heinrich, B. 1976a. Foraging specializations of individual bumblebees. Ecological Monitoring 46:105-128.

Heinrich, B. 1976b. Heat exchange in relation to blood flow between thorax and abdomen in bumblebees. Journal of Experimental Biology 64:561-585.

Heinrich, B. 1976c. Resource partitioning among some eusocial insects: Bumblebees. Ecology 57:874-889.

Heinrich, B. 1979. "Majoring" and "minoring" by foraging bumblebees, Bombus vagans: An experimental analysis. Ecology 60:245-255.

Heinrich, B. 1993. The Hot-blooded Insects: Mechanisms and Evolution of Thermoregulation. Harvard University Press, Cambridge, MA. 601 pp.

Heinrich, B. 1994. A Year in the Maine Woods. Addison and Wesley, Boston, MA. 304 pp.

Heinrich, B. 1995. Bumblebee ecology. Wild Earth Summer 1995:45-47

Heinrich, B., and G. Chavarria. 2001. Bumble bee energetics and conservation, In C.S. Stubbs and F.A. Drummond (Eds.). Pollination and Bee Conservation: A Cross Roads. Thomas Say Publications in Entomology, Entomological Society of America, Annapolis, MD. 156 pp.

Heinrich, B. 2004. Bumblebee Economics. 1979 Edition with New Preface. Harvard University Press, Cambridge, MA.

Heinrich, B., and M.J.E. Heinrich. 1983a. Size and caste in temperature regulation by bumblebees. Physiological Zoology 56(4):552-562.

Heinrich, B., and M.J.E. Heinrich. 1983b. Heterothermia in foraging workers and drones of the bumblebee Bombus terricola. Physiological Zoology 56(4):563-567.

Heinrich, B., P. Mudge, and P. Deringis. 1977. A laboratory analysis of flower constancy in foraging bumblebees: Bombus ternarius and B. terricola. Behavioral Ecology and Sociobiology 2:247-266.

Hinojosa-Díaz, I.A. 2008. The Giant Resin Bee making its way west: First record in Kansas (Hymenoptera: Megachilidae). ZooKeys 1:67-71. Available online at DOI:10.3897/ zookeys. 1.17

Hurd, P.D., Jr., and E.G. Linsley. 1972. Parasitic bees of the genus Holcopasites Ashmead (Hymenoptera: Apoidea). Smithsonian Contributions to Zoology No. 114. Smithsonian Institution Press, Washington, DC. 39 pp.

Javorek, S.K., K.E. Mackenzie, and S.P. Vander Kloet. 2002. Comparative pollination effectiveness among bees (Hymenoptera: Apoidea) on Lowbush Blueberry (Ericaceae: Vaccinium angustifolium). Annals of the Entomological Society of America 95(3):345-351.

Jones, M.S., H. Vanhanen, R. Peltola, and F.A. Drummond. 2014. A global review of arthropod-mediated ecosystem-services in Vaccinium berry agroecosystems. Terrestrial Arthopod Reviews 7:41-78.

Kerr J.T., A. Pindar, P. Galpern, L. Packer, S.G. Potts, S.M. Roberts, P. Rasmont, O. Schweiger, S.R. Colla, L.L. Richardson, D.L. Wagner, L.F. Gall, D.S. Sikes, and A. Pantoja. 2015. Climate change impacts on bumblebees converge across continents. Science 349(6244):177-180. Available online at DOI:10.1126/science.aaa7031.

Kingsbury, S.M. 1906. The records of the Virginia Company of London, Vol. 1-2. The Court Book, from the manuscript in the Library of Congress 1619-1622. Government Printing Office, Washington, DC. Available online at https://archive.org/details/recordsofvirgini02virguoft. Accessed 27 June 2017.

LaBerge, W.E. 1956. Revision of the bees of the genus Melissodes in North and Central America. Parts I, II. (Hymentoptera, Apidae). University of Kansas Science Bulletin 37-38:911-1194. 
A.C. Dibble, F.A. Drummond, C. Stubbs, M. Veit, and J.S. Ascher

LaBerge, W.E. 1961. A revision of the bees of the genus Melissodes in North and Central America. Part III (Hymenoptera, Apidae). University of Kansas Science Bulletin 42:283-663.

LaBerge, W.E. 1967. A revision of the bees of the genus Andrena of the Western Hemisphere. Part I. Callandrena. Bulletin of the University of Nebraska State Museum 7:1-316.

LaBerge, W.E. 1971. A revision of the bees of the genus Andrena of the Western Hemisphere. Part IV. Scrapteropsis, Xiphandrena, and Rhaphandrena. Transactions of the American Entomological Society 97:441-520.

LaBerge, W.E. 1973. A revision of the bees of the genus Andrena of the Western Hemisphere. Part VI. Subgenus Trachandrena. Transactions of the American Entomological Society 99:235-371.

LaBerge, W.E. 1977. A revision of the bees of the genus Andrena of the Western Hemisphere. Part VIII. Subgenera Thysandrena, Dasyandrena, Psammandrena, Rhacandrena, Euandrena, and Oxyandrena. Transactions of the American Entomological Society 103:1-143.

LaBerge, W.E. 1980. A revision of the bees of the genus Andrena of the Western Hemisphere. Part X. Subgenus Andrena. Transactions of the American Entomological Society 106:395-525.

LaBerge, W.E. 1985. A revision of the bees of the genus Andrena of the Western Hemisphere. Part XI. Minor subgenera and subgeneric key. Transactions of the American Entomological Society 111:441-567.

LaBerge, W.E. 1987. A revision of the bees of the genus Andrena of the Western Hemisphere. Part XII. Subgenera Leucandrena, Ptilandrena, Scoliandrena, and Melandrena. Transactions of the American Entomological Society 112:191-248.

LaBerge, W.E. 1989. A revision of the bees of the genus Andrena of the western hemisphere. Part XIII. Subgenera Simandrena and Taeniandrena. Transactions of the American Entomological Society 115:1-56.

LaBerge, W.E., and J.K. Bouseman. 1970. A revision of the bees of the genus Andrena of the Western Hemisphere. Part III. Tylandrena. Transactions of the American Entomological Society 96:543-605.

LaBerge, W.E., and D.W. Ribble. 1972. A revision of the bees of the genus Andrena of the Western Hemisphere. Part V. Subgenera Gonandrena, Geissandrena, Parandrena, Pelicandrena. Transactions of the American Entomological Society 98:271-358.

LaBerge, W.E., and D.W. Ribble. 1975. A revision of the bees of the genus Andrena of the Western Hemisphere. Part VII. Subgenus Euandrena. Transactions of the American Entomological Society 101:371-446.

Ladurner, E., J. Bosch, W.P. Kemp, and S. Maini. 2008. Foraging and nesting behavior of Osmia lignaria (Hymenoptera: Megachilidae) in the presence of fungicides: Cage studies. Journal of Economic Entomology 101(3):647-653.

Laycock, I., K.M. Lenthall, A.T. Barratt, and J.E. Cresswell. 2012. Effects of imidacloprid, a neonicotinoid pesticide, on reproduction in worker bumble bees (Bombus terrestris). Ecotoxicology 21(7):1937-1945.

Lee, K.V., N. Steinhauer, K. Rennich, M.E. Wilson, D.R. Tarpy, D.M. Caron, R. Rose, K.S. Delaplane, K. Baylis, E.J. Lengerich, J. Pettis, J.A. Skinner, J.T. Wilkes, R. Sagili, and D. vanEngelsdorp. 2015. A national survey of managed Honey Bee 2013-2014 annual colony losses in the USA. Apidologie 46(3):292-305. 
A.C. Dibble, F.A. Drummond, C. Stubbs, M. Veit, and J.S. Ascher

Lee, W.R. 1958. Pollination studies on Low Bush Blueberries. Journal of Economic Entomology 51:544-545.

Ley, E.L., S. Buchmann, K. McGuire, and L. Stritch. 2011. Selecting plants for pollinators: A regional guide for farmers, land managers, and gardeners in the ecological region of the Laurentian Mixed Forest Province. The Pollinator Partnership/North American Pollinator Project Campaign, San Francisco, CA. Available online at http://www.pollinator. org/PDFs/Laurentian.rx9.pdf. Accessed 27 June 2017.

Lovell, J.H. 1900. The visitors of the Caprifoliaceae. American Naturalist 34:37-51.

Lovell, J.H. 1905a. Four new species of Halictus from Maine. Canadian Entomologist 37:39-40.

Lovell, J.H. 1905b. Some Maine species of Halictus. Canadian Entomologist 37:299-300.

Lovell, J.H. 1907. The Colletidae of southern Maine. Canadian Entomologist 39:363-365.

Lovell, J.H. 1908. The Halictidae of southern Maine. Psyche 15:32-40.

Lovell, J. H. 1909. The bees of Massachusetts: Osmia and Sphecodes. Entomological New 20: $122-126$.

Lovell, J.H. 1910. The Prosopididae of southern Maine. Psyche 17(5):177-185.

Lovell, J.H. 1911. New records of bees: Sphecodes and Prosopis (Hym.) Entomological News 22:211-214.

Lovell, J.H. 1913. The origin of the oligotropic habit among bees (Hymenoptera). Entomological News 24:104-112.

Lovell, J.H. 1922a. The bees of Maine. The Maine Naturalist 4:71-76.

Lovell, J.H. 1922b. The bees of Maine. Part II. The Maine Naturalist 4:102-105.

Lovell, J.H. 1924. Pollination of Alfalfa. American Bee Journal 66:176-178.

Lovell, J.H. 1925a. The bees of Maine. Part III. The Maine Naturalist 5:7-10.

Lovell, J.H. 1925b. The bees of Maine. Part IV. The Maine Naturalist 5:57-60.

Lovell, J.H. 1925c. The bees of Maine. Part IV. The Maine Naturalist 5:111-115.

Lovell, J.H. and T.D.A. Cockerell. 1905. The nomadine and epeoline bees of southern Maine. Psyche 12:39-42.

Lovell, J.H. and T.D.A. Cockerell. 1906. Notes on the bees of southern Maine: Anthophoridae, Halictoididae, Macropidae, and Panurgidae. Psyche 13(5):109-113.

Lovell, J.H. and T.D.A. Cockerell. 1907a. The Megachilidae of southern Maine. Psyche $14: 15-21$.

Lovell, J.H. and T.D.A. Cockerell. 1907b. The Sphecodidae of southern Maine. Psyche 14(5):101-110.

Lovell, J.H., and H.B. Lovell. 1932. The pollination of Rhodora. Rhodora 36(407):213-214.

Maine Department of Agriculture, Conservation and Forestry. 2017. Invasive plant list. Available online at http://www.maine.gov/dacf/mnap/features/invasive_plants/invsheets.htm. Accessed 27 June 2017.

Martin, E.C., E. Oertel, and N.P. Nye. 1980. Beekeeping in the United States. Agricultural Handbook No. 335. US Department of Agriculture, Washington, DC. 193 pp.

Matteson, K.C., J.S. Ascher, and G.A. Langellotto. 2008. Bee richness and abundance in New York City urban gardens. Annals of the Entomological Society of America 101(1):140-150.

Mazurkiewicz, M. 2010. The Giant Resin Bee, Megachile sculpturalis, in Maine: A new state record. The Maine Entomologist 14(4):3.

McGinley, R.J. 1986. Studies of Halictinae (Apoidea: Halictidae), I: Revision of New World Lasioglossum Curtis. Smithsonian Contributions to Zoology No. 429, Smithsonian Institution Press, Washington, DC. 
A.C. Dibble, F.A. Drummond, C. Stubbs, M. Veit, and J.S. Ascher

Michener, C.D. 1947. A revision of the American species of Hoplitis (Hymenoptera, Megachilidae), Bulletin of the American Museum of Natural History 89:263-317.

Michener, C.D. 2007. Bees of the World. Second Edition. John Hopkins University Press, Baltimore, MD. 93 pp.

Miliczky, E.R. 1978. Effects of spraying with Sevin-4-oil ${ }^{\circledR}$ on the abundance of insect pollinators in the spruce-fir forest. M.Sc. Thesis. University of Maine, Orono, ME. 37 pp.

Miliczky, E.R. and E.A. Osgood. 1979a. The effects of spraying with Sevin-4-oil ${ }^{\circledR}$ on insect pollinators and pollination in a spruce-fir forest. Life Sciences and Agriculture Experiment Station Technical Bulletin 90. University of Maine, Orono, ME.

Miliczky, E.R., and E.A. Osgood. 1979b. Insects visiting bloom of Withe-rod, Viburnum cassinoides L., in the Orono, Maine, area. Entomological News 90(3):131-134

Miller-Rushing, A.J., and R.B. Primack. 2008. Global warming and flowering times in Thoreau's Concord: A community perspective. Ecology 89(2):332-341.

Mitchell, T.B. 1960. Bees of the Eastern United States. Volume 1: North Carolina Agricultural Experimental Station Technical Bulletin 141. Raleigh, NC. 538. pp.

Mitchell, T.B. 1962. Bees of the Eastern United States. Volume 2. North Carolina Agricultural Experiment Station Technical Bulletin 152. Raleigh, NC. 557 pp.

Moure, J.S., and P.D. Hurd Jr. 1987. An Annotated Catalog of the Halictid Bees of the Western Hemisphere (Hymenoptera: Halictidae). Smithsonian Institution Press, Washington, DC. 405 pp.

Neumann P., and N.L. Carreck. 2010. Honey Bee colony losses. Journal of Apicultural Research 49(1):1-6. Available online at doi:10.3896/IBRA.1.49.1.01.

Osgood, E.A. 1972. Soil characteristics of nesting sites of solitary bees associated with the Low-bush Blueberry in Maine. Life Science and Agricultural Experiment Station Bulletin 59. Orono, ME. 8 pp.

Osgood, E.A. 1989. Biology of Andrena crataegi Robertson (Hymenoptera: Andrenidae), a communally nesting Bee. Journal of the New York Entomological Society 97(1):56-64.

Ostaff, D.P., A. Mosseler, R.C. Johns, S. Javorek, J. Klymko, and J.S. Ascher. 2015. Willows (Salix spp.) as pollen and nectar sources for sustaining fruit- and berry-pollinating insects. Canadian Journal of Plant Science 95:505-516.

Packard, A.S., Jr. 1861. Report on the insects collected on the Penobscot and Alleguash Rivers, during August and September. Pp. 375-376, In Sixth Annual Report of the Secretary of the Maine Board of Agriculture, Augusta, ME.

Pellett, F.C. 1939. John H. Lovell: Notes on the life and writings of the Maine naturalist. American Bee Journal December 1939:568-570.

Phipps, C.R. 1930. Blueberry and huckleberry insects. Maine Agricultural Experiment Station Bulletin 356:107-232.

Pimentel, D., R. Zuniga, and D. Morrison. 2005. Update on the environmental and economic costs associated with alien-invasive species in the United States. Ecological economics 52(3):273-288.

Procter, W. 1938. Biological Survey of the Mount Desert Region. Part VI. The Insect Fauna. The Wistar Institute of Anatomy and Biology, Philadelphia, PA. 247 pp. Available online at https://www.biodiversitylibrary.org/page/5843840\#page/5/mode/1up. Accessed 27 June 2017.

Procter, W. 1946. Biological Survey of the Mount Desert Region. Part VII. The Insect Fauna. Being a revision of Parts I and VI with the addition of 1100 species. The Wistar Institute of Anatomy and Biology, Philadelphia, PA. 566 pp. Available online at https:// www.biodiversitylibrary.org/item/29157\#page/5/mode/1up. Accessed 27 June 2017. 
A.C. Dibble, F.A. Drummond, C. Stubbs, M. Veit, and J.S. Ascher

Ratnieks, F.W., and N.L. Carreck. 2010. Clarity on honey bee collapse? Science 327(5962):152-153.

Ribble, D.W. 1968. Revisions of two subgenera of Andrena: Micrandrena Ashmead and Derandrena, new subgenus (Hymenoptera: Apoidea). Bulletin of the University of Nebraska State Museum 8(5):237-394.

Rightmyer, M.G., T. Griswold, and M.S. Arduser. 2010. A review of the non-metallic Osmia (Melanosmia) found in North America, with additional notes on palearctic Melanosmia (Hymenoptera, Megachilidae). ZooKeys 60:37-77.

Rivernider, R., E. Venturini, and F. Drummond. In press. Timothy Grass: A pollen forage for bumble bees. Journal of the Kansas Entomological Society.

Roberts, R.B. 1972. Revision of the bee genus Agapostemon (Hymenoptera: Halictidae). The University of Kansas Science Bulletin 49(9):437-590.

Robertson, C. 1929. Flowers and insects: Lists of visitors of four hundred and fifty-three flowers. No publisher. Carlinville, Ill. Available online at http://dx.doi.org/10.5962/bhl. title.11538. Accessed October 2017.

Romey, W.L., J.S. Ascher, D.A. Powell, and M. Yanek. 2007. Impacts of logging on midsummer diversity of native bees (Apoidea) in a northern hardwood forest. Journal of the Kansas Entomological Society 80(4):327-338.

Roulston, T., and R. Malfi. 2012. Aggressive eviction of the Eastern Carpenter Bee (Xylocopa virginica (Linnaeus)) from its nest by the Giant Resin Bee (Megachile sculpturalis Smith). Journal of the Kansas Entomological Society 85(4):387-388.

Rust, R.W., and E.A. Osgood. 1993. Identification of Osmia kenoyeri and O. virga (Hymenoptera: Megachilidae), two blueberry pollinators. Entomological News 104:113-117.

Seltmann. 2013. Arthropod Easy Capture. Version: 1.34 (Date: 18 February 2013). Available online at http://sourceforge.net/p/arthropodeasy. Accessed 27 June 2017.

Schwarz, H.F. 1926. Observations on the bees of Rangeley, Maine. The Maine Naturalist $5: 136-148$.

Schwarz, M., and F. Gusenleitner. 2004. Beitrag zur klärung und kenntnis parasitärer bienen der gattungen Coelioxys and Nomada (Hymenoptera, Apidae). Linzer Biologische Beiträge 36(2):1413-1485.

Scott, V.L., J.S. Ascher, T. Griswold, and C.R. Nufio. 2011. Bees of Colorado. Natural History Inventory of Colorado, No. 23. University of Colorado Museum of Natural History, Boulder, CO. 112 pp.

Sheffield, C.S., and J.-M. Perron. 2014. Annotated catalogue of the bees described by Léon Provancher (Hymenoptera: Apoidea). The Canadian Entomologist 146(2):117-169.

Sheffield, C.S., P.G. Kevan, R.F. Smith, S.M. Rignuy, and R.E.L. Rogers. 2003. Bee species of Nova Scotia, Canada, with new records and notes on bionomics and floral relations (Hymenoptera: Apoidea). Journal of the Kansas Entomological Society 76(2):357-384.

Sheffield, C.S., C. Ratti, L. Packer, and T. Griswold. 2011. Leafcutter and mason bees of the genus Megachile Latreille (Hymenoptera: Megachilidae) in Canada and Alaska. Canadian Journal of Arthropod Identification 18:1-107.

Sheffield, C.S., A. Pindar, L. Packer, and P.G. Kevan. 2013. The potential of cleptoparasitic bees as indicator taxa for assessing bee communities. Apidologie 44:501-510.

Simberloff, D. 2013. Invasive Species: What Everyone Needs to Know. Oxford University Press, Oxford, UK. 352 pp.

Smit, B., S.E. Wall, and J. Wandel. 2007. Changing climate: Agricultural adaption in Canada. University of British Columbia Press, Vancouver, BC. 273 pp. 
A.C. Dibble, F.A. Drummond, C. Stubbs, M. Veit, and J.S. Ascher

Stephen, W.P. 1954. A revision of the bee genus Colletes in America North of Mexico (Hymenoptera, Colletidae). The University of Kansas Science Bulletin 36(1):149-527.

Strange, J.P., J.B. Koch, V.H. Gonzalez, L. Nemelka, and T. Griswold. 2011. Global invasion by Anthidium manicatum (Linnaeus) (Hymenoptera: Megachilidae): Assessing potential distribution in North America and beyond. Biological Invasions 13:2115-2133.

Stubbs, C.S., and F.A. Drummond. 1997a. Blueberry and cranberry (Vaccinium spp.) pollination: A comparison of managed and native bee foraging behavior. Proceedings of the International Symposium on Pollination. Acta Hort 437:341-343.

Stubbs, C.S., and F.A. Drummond. 1997b. Pollination of wild Lowbush Blueberry, Vaccinium angustifolium, by the Alfalfa Leafcutting Bee, Megachile rotundata. Proceedings of the Sixth International Symposium on Vaccinium Culture. Acta Hort 446:189-196.

Stubbs, C.S., and F.A. Drummond. 1997c. Management of the Alfalfa Leafcutter Bee, Megachile rotundata (Hymenoptera: Megachilidae), for pollination of wild Lowbush Blueberry. Journal of the Kansas Entomological Society 70(2):81-93.

Stubbs, C.S., and F.A. Drummond. 2001. Bombus impatiens (Hymenoptera: Apidae): An alternative to Apis mellifera (Hymenoptera: Apidae) for Lowbush Blueberry pollination. Journal of Economic Entomology 94(3):609-616.

Stubbs, C.S., H.A. Jacobson, E.A. Osgood, and F.A. Drummond. 1992. Alternate forage plants for native (wild) bees associated with lowbush blueberry (Vaccinium spp.) in Maine. University of Maine Agricultural Experiment Station Technical Bulletin 148. $54 \mathrm{pp}$.

Stubbs, C.S., E.A. Osgood, J.B. Dimond, and F.A. Drummond. 1996. Hymenoptera diversity in Maine. Pp. 81-86, In S.C. Gawler, J.J. Albright, P.D. Vickery, and F.S. Smith (Eds.). Biological Diversity in Maine. Maine Natural Areas Program, Maine Forest Biodiversity Project. Augusta, ME. [\# OF PP?].

Stubbs, C.S., F.A. Drummond, and S.L. Allard. 1997a. Bee conservation and increasing Osmia spp. in Maine wild blueberry fields. Northeast Naturalist 4(3):133-144.

Stubbs, C.S., F.A. Drummond, and D. Yarborough. 1997b. How to manage Alfalfa Leafcutting Bees for wild blueberry pollination. Wild Blueberry Fact Sheet No. 300. University of Maine Cooperative Extension Bulletin No. 2413. Orono, ME Available online at https:/extension.umaine.edu/blueberries/factsheets/bees/300-how-to-manage-alfalfaleafcutting-bees-for-wild-blueberry-production/. Accessed 27 June 2017.

Stubbs, C.S., F.A. Drummond, and D. Yarborough. 2001. Commercial bumble bee, Bombus impatiens, management for wild blueberry pollination. Wild blueberry fact sheet No. 302. University of Maine Cooperative Extension Bulletin No. 2421. Orono, ME. 4 pp. Available online at https://extension.umaine.edu/blueberries/factsheets/bees/302-commercial-bumble-bee-bombus-impatiens-management-for-wild-blueberry-pollination/. Accessed 27 June 2017.

Stubbs, C.S., F.A. Drummond, and H. Ginsberg. 2007. Effects of invasive plant species on pollinator service and reproduction in native plants at Acadia National Park. Technical Report NPS/NER/NRTR - 2007/096. Available online at https://pubs.er.usgs.gov/publication/5200339. Accessed 27 June 2017.

Torchio, P.F. 1976. Use of Osmia lignaria Say (Hymenoptera: Apoidea, Megachilidae) as a pollinator in an apple and prune orchard. Journal of the Kansas Entomological Society 49(4):475-482.

Tsvetkov, N., O. Samson-Robert, K. Sood, H.S. Patel, D.A. Malena, P.H. Gajiwala, P. Maciukiewicz, V. Fournier, and A. Zayed. 2017. Chronic exposure to neonicotinoids reduces honey bee health near Corn crops. Science 356:1395-1397. 
A.C. Dibble, F.A. Drummond, C. Stubbs, M. Veit, and J.S. Ascher

vanEngelsdorp, D., and M. Meixner. 2010. A historical review of managed honey bee populations in Europe and the United States and the factors that may affect them. Journal of Invertebrate Pathology 103:580-595.

Venturini, E.M, L. Berg Stack, A.C. Dibble, F.A. Drummond, and A.K. Hoshide. 2015. Enhancing wild bees for crop pollination: Sowing bee pasture for New England's wild Lowbush Blueberry. University of Maine Cooperative Extension Fact Sheet, Orono, ME. 9 pp. Available online at https://extension.umaine.edu/blueberries/wp-content/ uploads/sites/56/2010/05/2015-Bee-Pasture-Fact-Sheet.pdf. Accessed 27 June 2017.

Venturini, E.M, F.A. Drummond, A.K. Hoshide, A.C. Dibble, and L. Berg Stack. 2017. Pollination reservoirs in lowbush blueberry (Ericales: Ericaceae). Journal of Economic Entomology 110(2):333-346.

Viereck, H.L. 1922. New bees of the genus Andrena. Occasional papers of the Boston Society of Natural History 5:35-45, pl. 4.

Wagner, D.L., and J.S. Ascher. 2008. Rediscovery of Epeoloides pilosula (Cresson) (Hymenoptera: Apidae) in New England. Journal of the Kansas Entomological Society 81:81-83.

Wagner, D.L., J.S. Ascher, and N.K. Bricker. 2014. A transmission right-of-way as habitat for wild bees (Hymenoptera: Apoidea: Anthophila) in Connecticut. Annals of the Entomological Society of America 107(6):1110-1120.

Wcislo, W.T. 1996. Parasitism rates in relation to nest site in bees and wasps (Hymenoptera: Apoidea). Journal of Insect Behavior 9(4):643-656.

Weber, E. 2017. Invasive Plant Species of the World: A Reference Guide to Environmental Weeds. Second Edition. Centre for Agriculture and Biosciences International Publications, Wallingford, UK.

Wessels, T. 1997. Reading the Forested Landscape: A Natural History of New England. Countryman Press, Woodstock, VT. 200 pp.

Williams, P.H., R.W. Thorp, L.L. Richardson, and S.R. Cola. 2014. Bumble Bees of North America: An Identification Guide (Princeton Field Guides). Princeton University Press, Princeton, NJ. 311 pp.

Wilson, E.H., and S.A. Sader. 2002. Detection of forest harvest type using multiple dates of Landsat TM imagery. Remote Sensing of Environment 80(3):385-396.

Wolf, A.T., and J.S. Ascher. 2009. Bees of Wisconsin (Hymenoptera: Apoidea: Anthophila). The Great Lakes Entomologist 41(1,2):129-168.

Woodcock, B.A., J.M. Bullock, R.F. Shore, M.S. Heard, M.G. Pereira, J. Redhead, L. Ridding, H. Dean, D. Sleep, P. Henrys, J. Peyton, S. Hulmes, L Hulmes, M. Sárospataki, C. Saure, M. Edwards, E. Genersch, S. Knäbe, and R.F. Pywell. 2017. Country-specific effects of neonicotinoid pesticides on honey bees and wild bees. Science 356:1393-1395.

Yamada, M., N. Oyama, N. Sekiga, S. Shirasaki, and C. Tsugawa. 1971. The ecology of the megachillid bee Osmia cornifrons (Radoszkowski) (Hymenoptera: Apidae) and its utilization for Apple pollination. Bulletin of the Aomori Apple Experiment Station, No. 15. Aomori, Japan.

Yarborough, D. 2009. Wild blueberry culture in Maine. University of Maine Cooperative Extension Fact Sheet No. 220. UMaine Extension Bulletin No. 2088. Orono, ME. 4 pp. Available online at http://extension.umaine.edu/blueberries/factsheets/production/wildblueberry-culture-in-maine/. Accessed 27 June 2017. 